\title{
RADIO DIRECTION FINDING NETWORK RECEIVER DESIGN FOR LOW-COST PUBLIC SERVICE APPLICATIONS
}

\author{
A Thesis Presented to the Faculty of \\ California Polytechnic State University, San Luis Obispo
}

\author{
In Partial Fulfillment \\ of the Requirements for the Degree \\ Master of Science in Electrical Engineering
}

By

Marcel Colman Eric Stieber

December 2012 


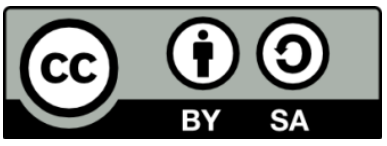

This work is licensed under the Creative Commons Attribution-ShareAlike 3.0 Unported License. This license lets others remix, tweak, and build upon this work even for commercial purposes, as long as they credit the author and license their new creations under the identical terms. To view a copy of this license, visit http://creativecommons.org/licenses/by-sa/3.0/ or send a letter to Creative Commons, 444 Castro Street, Suite 900, Mountain View, California, 94041, USA. 


\section{COMMITTEE MEMBERSHIP}

TITLE:

DATE SUBMITTED:

COMMITTEE CHAIR:

COMMITTEE MEMBER:

COMMITTEE MEMBER:
Radio Direction Finding Network Receiver Design for Low-cost

Public Service Applications

Marcel Colman Eric Stieber

December 2012

Dr. Clark Savage Turner J.D., Professor of Computer Science

Dr. Dennis J. Derickson, Professor of Electrical Engineering

Dr. John A. Saghri, Professor of Electrical Engineering 


\title{
ABSTRACT \\ RADIO DIRECTION FINDING NETWORK RECEIVER DESIGN FOR LOW-COST PUBLIC SERVICE APPLICATIONS
}

\author{
Marcel Colman Eric Stieber
}

A low-cost radio direction finding (RDF) VHF receiver has been investigated for development into a radio direction finding network (RDFN) with a particular focus towards public service and commercial asset tracking applications. The primary design criteria were reproducibility, low-cost, and simplicity such that public service and volunteer organizations can benefit from the technology. Two receiver designs were built and tested to allow for comparison of practicality, cost, and accuracy. A pseudo-Doppler RDF and a time difference of arrival (TDOA) receiver were built as proof-of-concept for a system design based on commercial off-the-shelf (COTS) components. The pseudo-Doppler system is a less practical implementation due to the necessity for custom hardware, a large antenna system, and an increased directional error due to multipath and weak signals. The TDOA system has potential as a very simple and low-cost RDFN implementation, but requires extremely accurate time synchronization that is difficult to achieve using COTS GPS receiver modules. The final proposed solution takes advantage of the simple TDOA hardware and multiple detection techniques (including signal strength) to produce improved locational data and ultimately provide a more accurate estimate of position. Further development and improvements to this receiver design have the potential for implementation as a low-cost radio direction finding network.

Keywords: Radio Direction Finding Network, Time Difference of Arrival, Pseudo-Doppler, Time of Arrival, Signal Strength, GPS, Clock Drift, Bias, and Accuracy. 


\section{Acknowledgements}

Countless people have contributed to my thesis both through direct support and in spirit. My thesis advisor, Dr. Clark Savage Turner WA3JPG, offered to step outside the realm of the computer science department and share his expertise in antennas and radio systems. Dr. Dennis Derickson AC0P, Electrical Engineering Department Chair, has been instrumental in supporting my education and Amateur Radio activities at Cal Poly which ultimately lead to the development of this project. Thank you to Dr. John Saghri, Professor of Electrical Engineering, serving as a member of my thesis committee. I also want to acknowledge my Amateur Radio Elmers (mentors), Ron Patterson W6FM and John Rogers KK6DJ, for their motivation and knowledge throughout the past 4 years, and my many peers and classmates that have helped with brainstorming, testing prototypes, and proofreading. Lastly and most importantly, a thank you to my parents, Hella and Tony, and my sister, Chantal, for their continued support, high expectations, and daily inspiration to do my best.

-Marcel Stieber, AI6MS 


\section{Table of Contents}

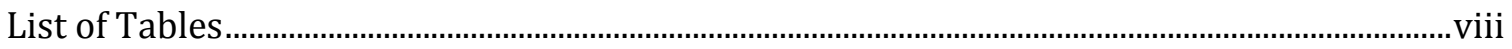

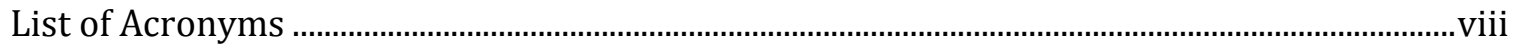

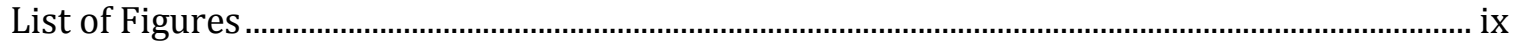

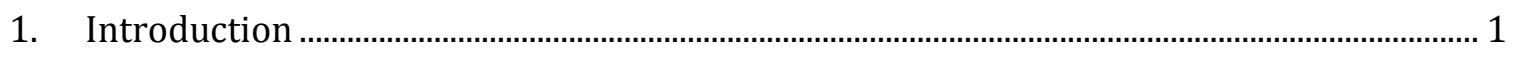

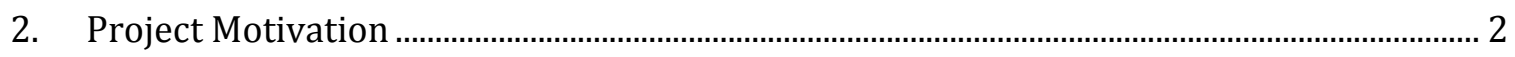

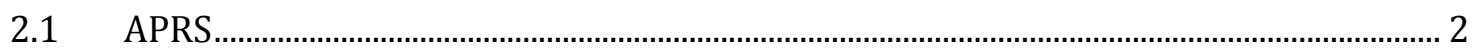

2.2 Beyond APRS: Public Service Applications ........................................................................... 3

3. Radio Direction Finding Overview........................................................................................ 6

4. Existing Technologies and Implementations................................................................................. 7

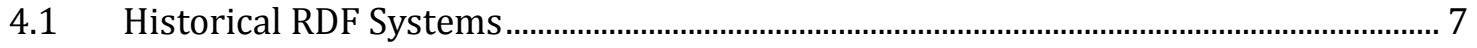

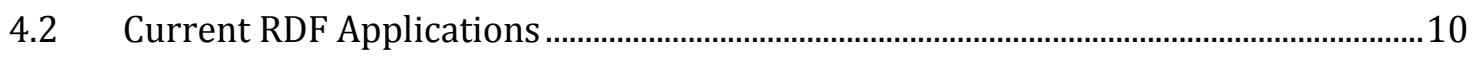

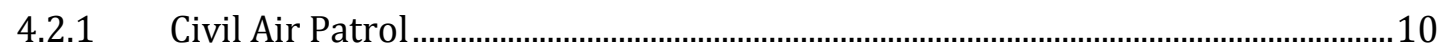

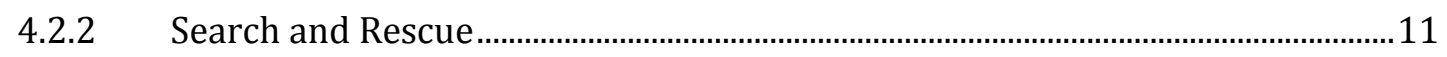

4.2.3 Wildlife Tracking................................................................................................... 12

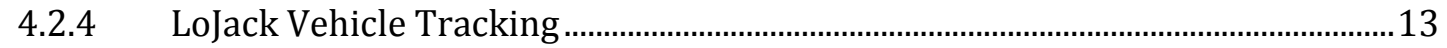

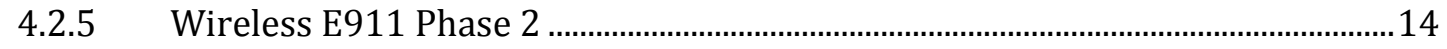

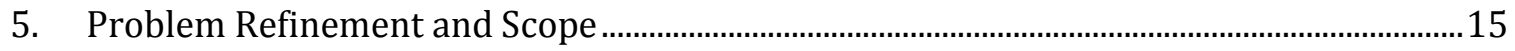

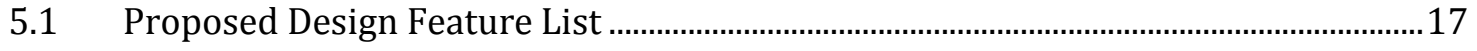

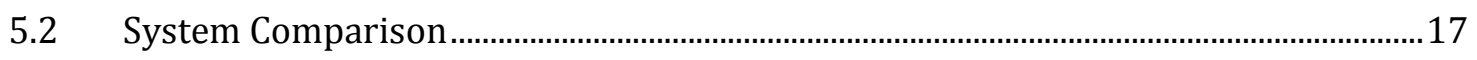

5.2.1 TDOA

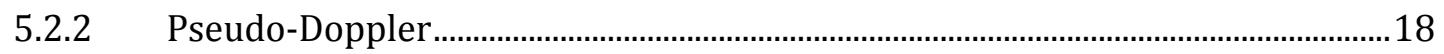

6. Pseudo-Doppler Direction Finding Prototype .......................................................................19

6.1 Pseudo-Doppler Direction Finding Theory of Operation..................................................20

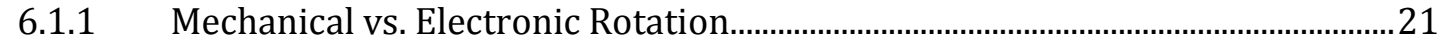

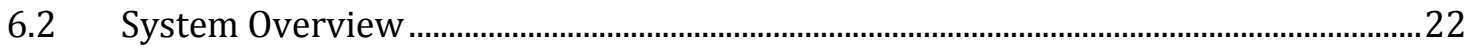

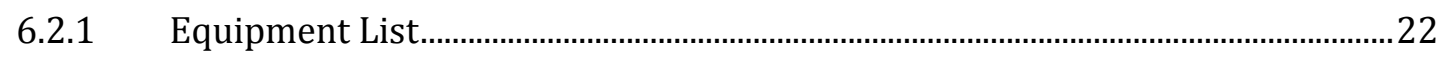

6.3 System Design Modification and Construction ...............................................................23

6.3.1 Antenna Switcher Design and Construction............................................................... 23

6.3.2 Antenna Switching Control........................................................................................

6.4 Pseudo-Doppler RDF System Setup and Testing................................................................

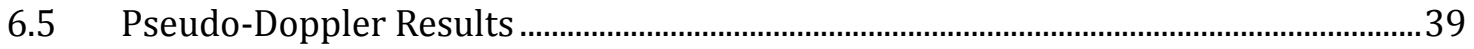

6.6 Conclusion on the Pseudo-Doppler Receiver......................................................................... 40

7. Time Difference of Arrival Direction Finding Prototype .......................................................... 41

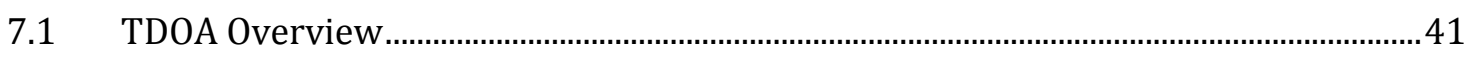

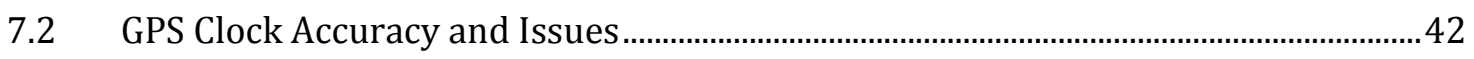




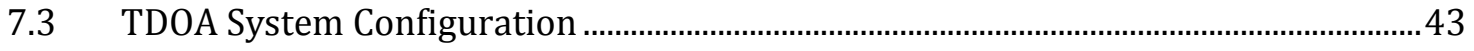

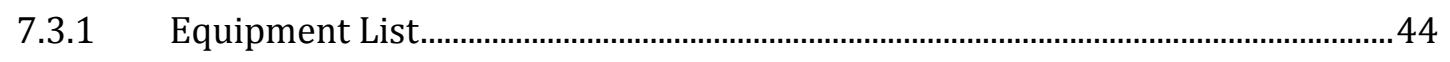

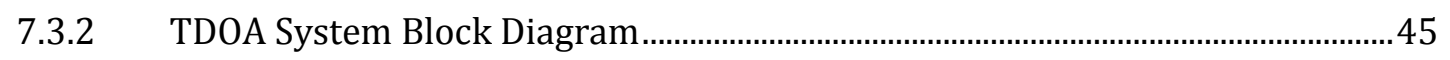

7.4 TDOA System Component Validation ..............................................................................46

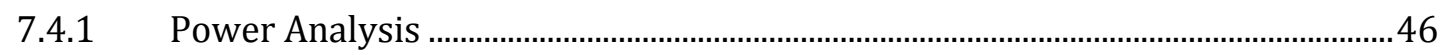

7.4.2 Friendcom FC-301/D VHF Transmitter Characterization .........................................4

7.5 T3-301 APRS Module Configuration …………....................................................................50

7.5.1 T3-301 Configuration Software ................................................................................. 51

7.5.2 T3-301 Device Interfacing................................................................................ 52

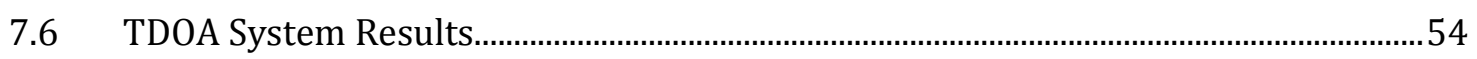

7.6.1 APRS Packet Results ....................................................................................................54

7.6.2 COTS Hardware Implementation ...................................................................................55

7.6.3 COTS Timing Challenges ........................................................................................ 55

7.6.4 TDOA Network Implementation.............................................................................. 56

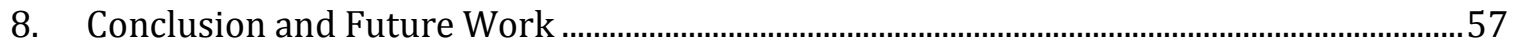

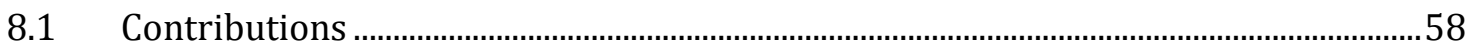

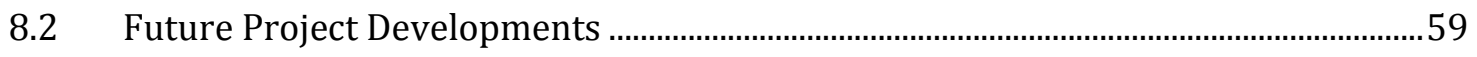

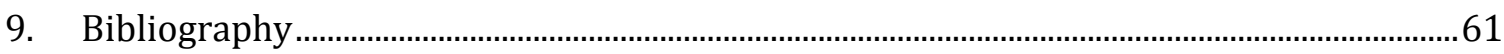

10. Appendix A: Arduino Antenna Switcher Code .........................................................................66

11. Appendix B: NMEA 0183 GGA Packet Format [57][58][60] ……….....................................68

12. Appendix C: Argent Data Systems T3-301 Schematic [56] .....................................................69

13. Appendix D: Antenna Switcher Characterization ....................................................................... 70 


\section{List of Tables}

Table 1: Comparison of Proposed VHF RDF Network Technologies................................................17

Table 2: TDOA System Power Budget Analysis .......................................................................................... 46

Table 3: FC-301/D Data Radio Characterization ................................................................................... 48

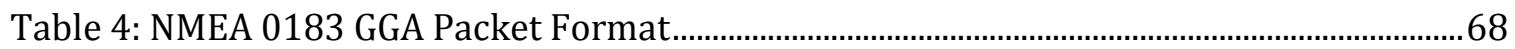

\section{List of Acronyms}

- APRS - Automatic Position (Packet) Reporting System

- ARES - Amateur Radio Emergency Services

- COTS - Commercial Off-The-Shelf

- DF - Direction Finding

- ELT - Emergency Locator Transmitter

- E-OTD - Estimated Observed Time Different

- GPS - Global Positioning System

- $\quad$ NDRS - National Distress and Response System

- NMEA - National Maritime Electronics Association

- RACES - Radio Amateur Civil Emergency Services

- $\quad$ RDF - Radio Direction Finding

- RDFN - Radio Direction Finding Network

- $\quad \mathbf{R F}$ - Radio Frequency

- $\quad$ SINAD - SIgnal to Noise And Distortion ratio

- SMD - Surface Mount Device

- TDOA - Time Difference of Arrival

- U-TDOA - Uplink Time Difference of Arrival

- $\quad$ VHF - Very High Frequency 


\section{List of Figures}

Figure 1: A modern H-Dipole Adcock DF Antenna for 20-174MHz[11] ........................................ 7

Figure 2: HFDF "Elephant Cage" Antenna Array[12] .............................................................................. 8

Figure 3: A portable Rescue 21 DF Tower with the Adcock DF array [15]................................... 9

Figure 4: L-Tronics Little L-Per Portable Direction Finder[17] ...................................................10

Figure 5: Backcountry Access Tracker DTS Avalanche Beacon [19] ...........................................11

Figure 6: A 170 milligram radio tracking tag on a bee [21] ..........................................................12

Figure 7: A manual VHF direction finding antenna system for wildlife tracking [22] .............12

Figure 8: LoJack vehicle tracking control head unit [23] .................................................................13

Figure 9: PicoDopp MiniMount Antenna Array [30] …………………..............................................18

Figure 10: A pseudo-Doppler RDF system by Mike Kossor WA2EBY [31] ...................................19

Figure 11: (A) A rotating monopole antenna and (B) the resultant Doppler frequency

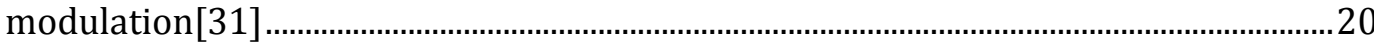

Figure 12: Pseudo-Doppler RDF system block diagram....................................................................22

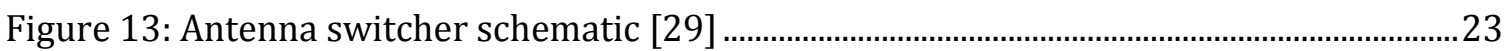

Figure 14: Improved antenna switcher schematic ...........................................................................24

Figure 15: Graph of 1N4148 diode isolation characteristics under biased and non-biased

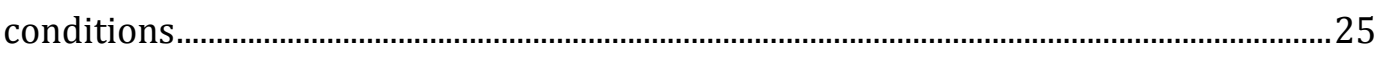

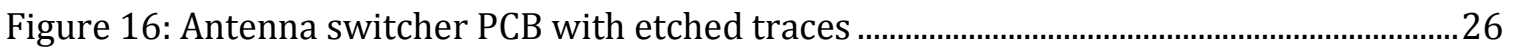

Figure 17: Populated antenna switcher PCB.........................................................................................26

Figure 18: Completed antenna switching board (using the Improved antenna switcher

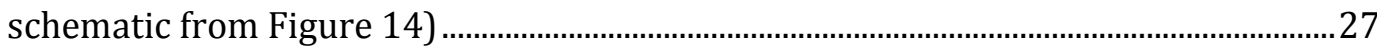

Figure 19: Close-up of Antenna Port 1 and Fm Receiver Port of the Antenna Switcher..........28

Figure 20: Antenna 1 DC forward-biased detail diagram ..................................................................29

Figure 21: Antenna 1 DC forward-biased RF path diagram ...........................................................29

Figure 22: Antenna 1 non-biased antenna isolation condition .........................................................

Figure 23: Simplified antenna switching control circuit schematic [29] .......................................31

Figure 24: Discrete component antenna switching control circuit.................................................31

Figure 25: Discrete component antenna switching control signals................................................32

Figure 26: Arduino generated antenna switching control signals .....................................................33

Figure 27: Arduino UNO controller antenna switching tests ............................................................34

Figure 28: VX-5R demodulated FM audio output FFT (250Hz per horizontal division) .........35

Figure 29: Pseudo-Doppler system test equipment configuration .................................................37 
Figure 30: Pseudo-Doppler RDF test system vehicle installation

Figure 31: SoundDoppler screenshot of a 147.36 MHz FM repeater on Mt Lowe. 38

Figure 32: SoundDoppler screenshot of a $146.5 \mathrm{MHz}$ local transmitter with multipath

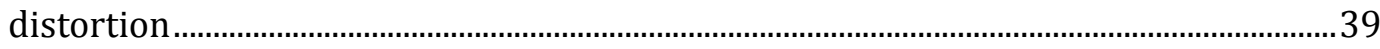

Figure 33: Complete COTS TDOA system with radio, battery, and GPS .......................................44

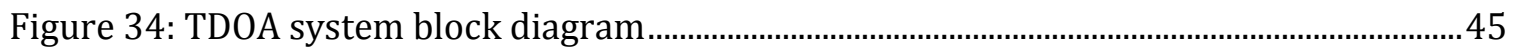

Figure 35: Genesis 12V 12Ah sealed lead-acid battery.....................................................................46

Figure 36: Friendcom FC-301/D VHF data radio ...........................................................................4 47

Figure 37: FC-301/D transceiver output spectrum at $144.990 \mathrm{MHz}$ (1 watt) ............................49

Figure 38: Argent Data Systems T3-301 Tracker Module v1.1 ………….....................................50

Figure 39: OTWINCFG T3-301 Configuration Utility ………….........................................................51

Figure 40: Squelch to IRQ jumper wire on the back of the T3-301.................................................52

Figure 41: OTWINCFG Script Editor ……………................................................................................53

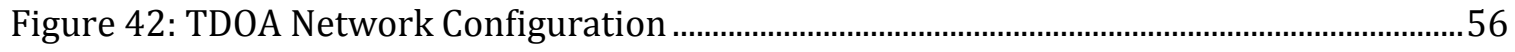

Figure 43: Antenna switcher characterization test configuration …………....................................70

Figure 44: Antenna switcher S21 transmission parameters with 0V diode bias........................71

Figure 45: Antenna switcher S21 transmission parameters with $1 \mathrm{~V}$ diode bias ........................72 


\section{Introduction}

Emergency services, including search and rescue teams, use radio direction finding (RDF) as a means to locate ambulances, emergency personnel, and disaster victims. A major challenge with these systems is that they are expensive and complicated, often requiring extensive infrastructure and training to implement. Volunteer and public service organizations are always struggling with shrinking budgets and aging equipment so the desire for a low-cost RDF system becomes ever stronger. Having a system that interoperates with existing equipment owned by the organization allows for lower costs and simplified implementation.

Radio direction finding networks (RDFN) are historically large, complex, and of high-cost. Attempts to miniaturize the systems or decrease their costs is often countered with reliability and performance challenges. The rapid development of RDF techniques and technologies in the past 60 years has greatly improved locating capabilities for these systems [1]. Improvements in GPS accuracy, radio transceiver technology, and availability of inexpensive precision measurement equipment have brought RDF into widespread use. What used to be a highly-expensive technology used primarily by the military is now implemented in countless civilian applications. RDFNs have expanded into daily use in cell phone locating for E-911 [2], patient tracking in hospitals, asset tracking for commercial applications, and stolen vehicle recovery [3].

As technology improves and equipment costs continue to decrease, the feasibility of a lowcost RDFN can become a reality. What is typically an expensive and complex commercial system can be realized in a consumer-level system made from low-cost off-the-shelf components. This report focuses on research and design of a low-cost RDFN system though analysis and system comparison of several different implementation techniques. 


\section{Project Motivation}

One of the motivating factors for this project comes from the Amateur Radio community and a project known as APRS or Automatic Position Reporting System [4]. APRS is an international standard used for positioning, messaging, and telemetry. Amateur radio operators world-wide have APRS enabled radios in their cars used for tracking their positions on the larger APRS network. This network is comprised of individual transmitters that are the end user in addition to mountain-top relay stations called digipeaters (digital repeaters) and iGates (internet gateways) to upload any received data to the online APRS network. Weather stations and APRS transmitter data can then easily be viewed online through sites like APRS.fi, offline through programs like AGW Tracker, or on a mobile device using programs similar to APRS Droid [5].

\subsection{APRS}

One of the primary attractions of the APRS system is that is it entirely volunteer supported by a network of enthusiastic radio operators world-wide. By making the initial project implementation compatible with the APRS network, one instantly obtains free technical support and system integration through the amateur radio community. Amateur radio organizations such as ARES and RACES (Amateur Radio Emergency Services and Radio Amateur Civil Emergency Services) are groups that are already integrated into public service disaster response and are a perfect test bed for a RDFN deployment. These groups regularly train with public agencies including police, fire, sheriff, and ambulance services. In designing a system for ARES and RACES, cost and simplicity are major factors to success of the system. Developing an RDFN that is both effective and affordable to these groups becomes the primary challenge and motivation for this project. 
For this project, the RDF receiver will be built from low-cost commercial radio components and designed for direct integration into the APRS network. By linking the RDFN with APRS, networking multiple receivers and relaying the collected information to a central location becomes trivial. Each individual receiver can simultaneously function as a digital repeater node for the network and transmit other stations' signals back to the central processing location [6].

The central processing for this proof of concept will initially be done manually through received packet data from each site, but future developments could incorporate the automated direction finding capabilities of the APRS network through specific packet design and implementation.

\subsection{Beyond APRS: Public Service Applications}

This project focus is to prove the feasibility of a low-cost, deployable, radio direction finding network that is made from Commercial Off-the-Shelf (COTS) components. The goal is to create an RDFN system that is within the practical reach for groups including Search and Rescue teams and Wildland Firefighters and made from COTS components. These organizations would benefit greatly from financially accessible RDFN systems with the stability and durability to be deployed in both temporary and long-term disaster relief. In Civil Air Patrol Search and Rescue, a mountain-top RDFN that monitors the National ELT (Emergency Locating Transmitter) frequency which is used for locating downed aircraft. This RDFN could provide real-time locating service to improve land-based response times and reduce the false-positives that plague around $97 \%$ of the older $121.5 \mathrm{MHz}$ ELT activations [7]. The newer satellite-based ELT system on $403 \mathrm{MHz}$ provides much better primary locating services within 2-3 miles from the initial beacon, but would still benefit 
greatly from an additional local RDFN that could pinpoint false alarms, particularly in cases where transmitters are accidentally activated in aircraft on the ground at an airport.

The RDFN developed in this project could also have major applications for Wildland Firefighters in large-scale deployments. Since each firefighter is already outfitted with a standard-issue VHF radio, an RDFN could easily maintain real-time locations on any radio users in the disaster area. Given the simplicity of the receiver design, individual RDF receivers could be deployed via aircraft in high-risk areas of a fire zone to establish the RDFN. These RDF receivers could also be easily upgraded to include environmental monitoring equipment to support firefighting operations with wind and temperature data metrics. The benefit of this system is that it can easily be integrated into existing systems since the only additional hardware is the RDFN itself. Anytime firefighters use their standard-issue radios to communicate, they would automatically be located and centrally logged to help with fire response and safety. Integration with radio fingerprinting systems and unique identifiers could allow for more extensive asset tracking in these scenarios.

An RDFN system would have been useful in a 2004 fire near Toulumne River Canyon when a fire flare-up caused the death of a firefighter who was building a fire break with her crew. In the incident report and following investigation, CDF Battalion Chief Dan Ward indicated that "it was not clear... where the crew was at the time of the flare up" [8]. "Water drops could have been requested...to support their fire line construction" and potentially have saved Firefighter Eva Schicke's life if the Battalion Chief had been better aware of the crew's location in the canyon.

Further analysis quickly reveals additional applications for this system design including locating of disaster workers, public service personnel, and commercial users. Typical asset 
tracking systems require expensive individual transmitters for each user, whereas an RDFN tracking system only requires the RDFN itself and no additional equipment for each end user. Another benefit from a deployed RDFN includes the ability to have multiple telemetry sites that can aid in disaster response. It becomes trivial to add weather sensors to a RDF receive site which can then transmit vital environmental data back to the central hub in addition to locating information. Alternately, RDF receivers can be deployed at existing NOAA weather sites around the USA at very low cost (due to existing infrastructure) and then provide immediate RDFN coverage for areas affected by the disaster. In either implementation, the existing networking infrastructure behind the system easily handles any additional data that needs to travel through the network for asset tracking capabilities. 


\section{Radio Direction Finding Overview}

Radio Direction Finding is designed to locate radio transmission sources. In the simplest abstraction, RDF is a two step process: receiving and characterizing a signal followed by processing the collected data. Receiving of a signal is typically done using a radio system designed to receive the frequency and modulation type of an incoming signal. Characterization of this signal can include gathering a number of signal metrics which typically include signal strength (commonly know as RSSI or Received Signal Strength Indicator), signal direction, and time of arrival. Each of these metrics provides a piece of the puzzle that can be used to locate the transmission source. For example, high signal strength and short time of arrival can indicate a signal's proximity to the receiver's location, and signal direction provides a heading indication of where the signal was received from. These measurements all aid in creating a complete model for signal locating.

The second part of the RDF process is data processing to calculate an approximate source location. In a Radio Direction Finding Network, a central processing site typically gathers data from multiple receive sites and then determines the most probable location of the signal

source. Based on the accuracy of each receive site's data and the method of direction finding used, the most probable location is then calculated. Typically, this last step is done on a computer and can easily be automated. Additional features are straightforward to implement in software and to integrate with existing systems and hardware. 


\section{Existing Technologies and Implementations}

\subsection{Historical RDF Systems}

Radio Direction Finding has practical origins in the early 1900's when Fred Adcock patented the 4-element Adcock antenna array that was used for discerning the direction of a radio signal [9]. RDF systems saw dramatic use in WWII where the High Frequency Direction Finding (HFDF or Huff-Duff) took off as a very important tool to detect submarines [10]. British and American Ships were outfitted with Adcock aerial antennas to help defend against the German "wolf pack" submarine attacks. By early detection of submarine communication signals and locations, ships were able to improve their preparations and response to submarine attacks. The widespread use of these systems is attributed to countless victories of the US and sunken German submarines in the 1940's.

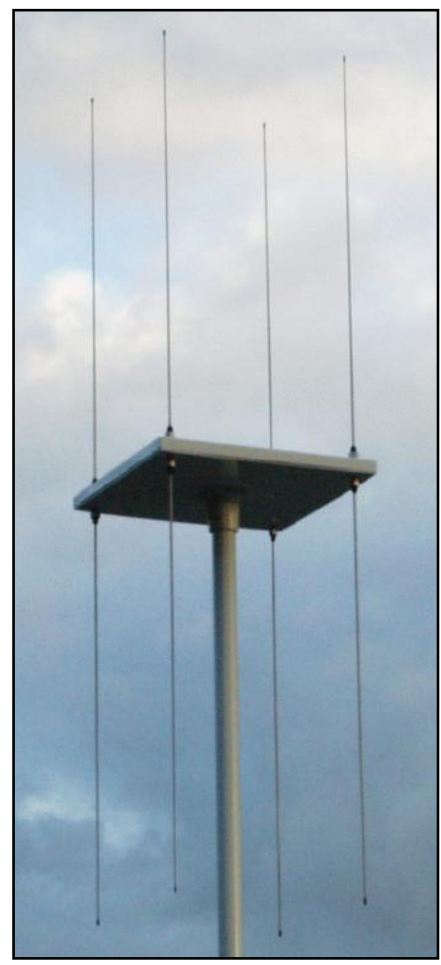

Figure 1: A modern H-Dipole Adcock DF Antenna for 20-174MHz[11] 
Ship-based DF systems used during World War II were very useful but the most impressive system developed is the Wide-Aperture Direction Finding (WADF) Circularly Disposed Antenna Array (CDAA) [9] shown in Figure 2. These enormous antennas arrays contain dozens of monopoles arranged in a circle with diameters ranging from $50 \mathrm{~m}$ to over $150 \mathrm{~m}$. Such systems used a capacitive switching goniometer to electronically select each antenna and produce highly-accurate azimuthal headings with sub-1-degree precision. These arrays were placed throughout the United States and networked extensively to provide accurate headings for any high frequency radio signals detected. These CDAA systems were expensive and very complicated, often accompanied by several buildings for control and processing equipment.

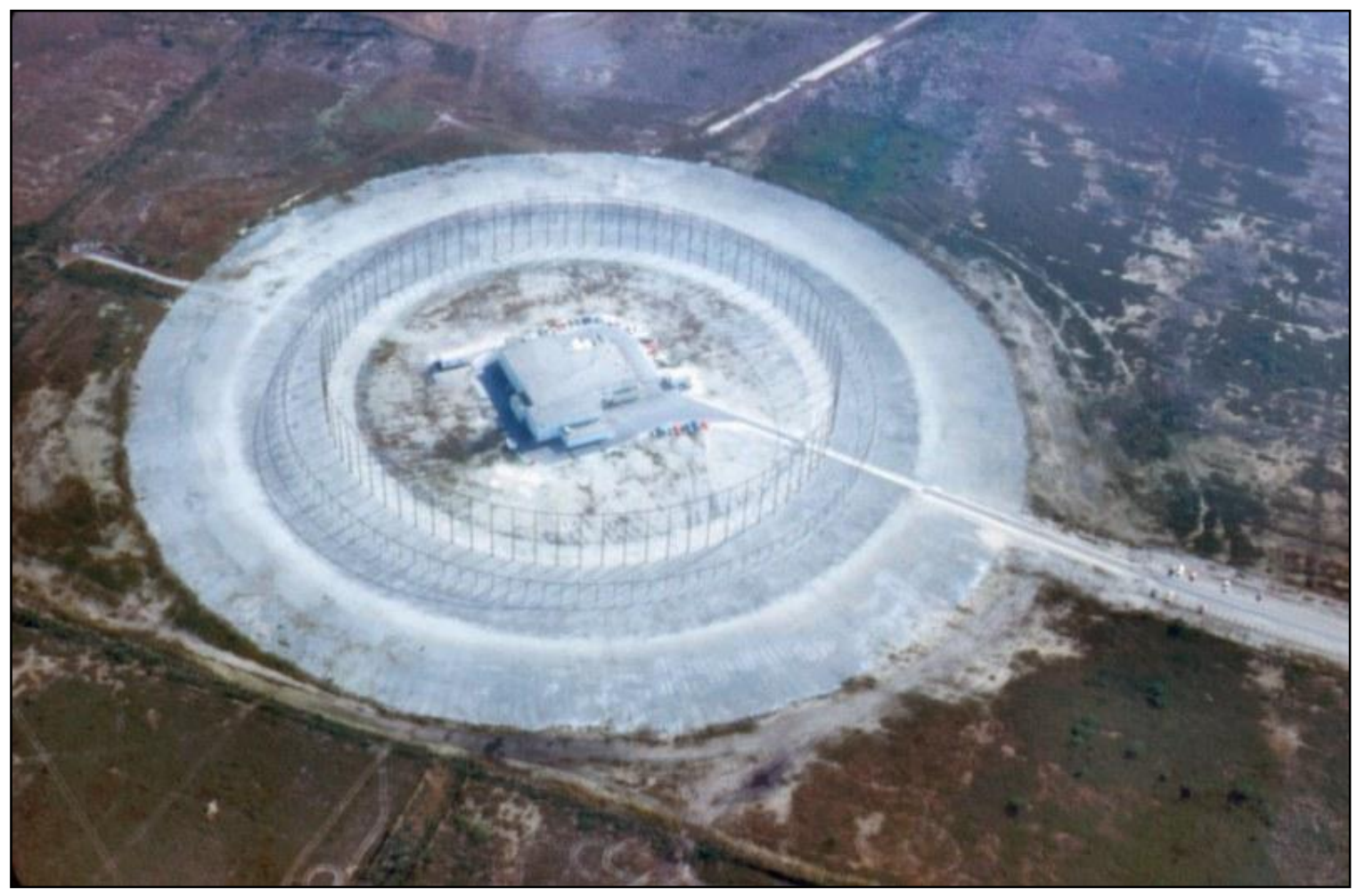

Figure 2: HFDF "Elephant Cage" Antenna Array[12] 
In 1970, the United States Coast Guard developed the National Distress System (NDS) which consists of over $300 \mathrm{VHF}$ radio receive sites along the US shorelines. These high-level sites monitor Marine Distress Channel 16 and cover up to 20 nautical miles from any shoreline including all the inland waterways in the nation [13]. The receivers are linked through a network of 44 communications centers around the US that continually monitor the system to provide immediate response to any marine distress call. The NDS is in the process of being upgraded to a much more sophisticated system called Rescue 21. This network uses VHF antenna systems with basic direction finding capabilities (Figure 3) as well as Digital Selective Calling (DSC) to allow both GPS and vessel information to be transmitted by the party in distress and provide more accurate and timely emergency response [14]. The primary disadvantage to the improved system is its vastly higher installation cost and high system complexity due to a complicated network infrastructure and integration.

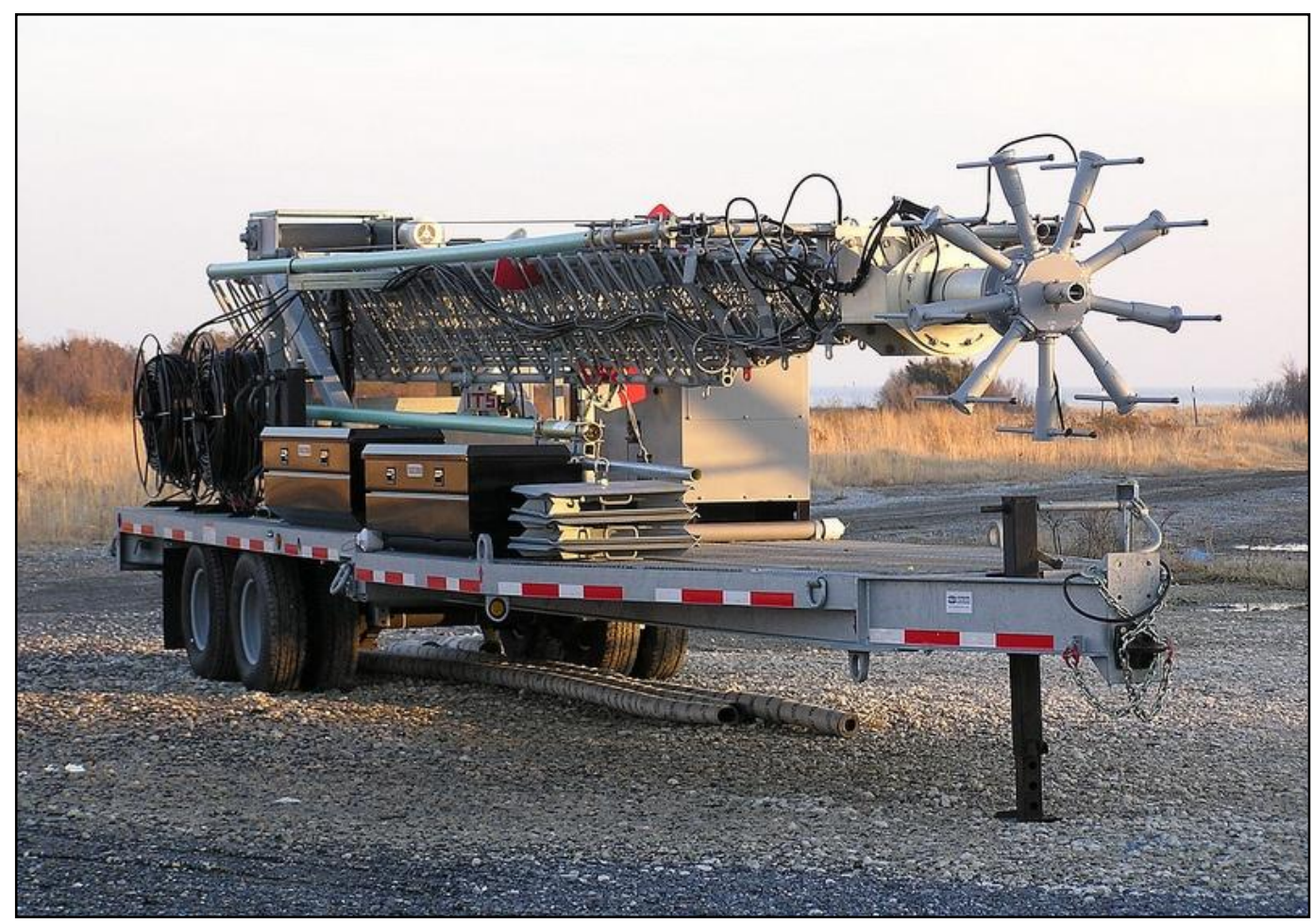

Figure 3: A portable Rescue 21 DF Tower with the Adcock DF array [15] 


\subsection{Current RDF Applications}

In this section, a number of existing RDF applications are introduced and characterized by their DF methods and system topology.

\subsubsection{Civil Air Patrol}

Civil Air Patrol (CAP) is an Auxiliary to the United States Air Force and provides Search and Rescue support for over $85 \%$ of all federal rescue missions from the Air Force Rescue Coordination Center [16]. CAP takes advantage of the Aircraft ELTs that operate on 121.5 and $406 \mathrm{MHz}$ to located downed or missing aircraft. The current system uses digital beacons on $406 \mathrm{MHz}$ that are monitored by a network of Search and Rescue satellites and activate the response system. Some of the newer beacons transmit GPS location data and a unique aircraft identifier to help with the rescue. Aircraft often have a $121.5 \mathrm{MHz}$ AM homing beacon onboard the plane that is activated during a crash and serves to help rescue teams locate the crash site. After initial detection, satellites are able to use Doppler-shift detection to localize the beacon within a 12-15 nautical mile accuracy. Highly-trained ground teams are then deployed to the search area and use handheld direction finding equipment (similar to that shown in Figure 4 ) to find the low-power ( 0.1 watt) transmitter on the aircraft.

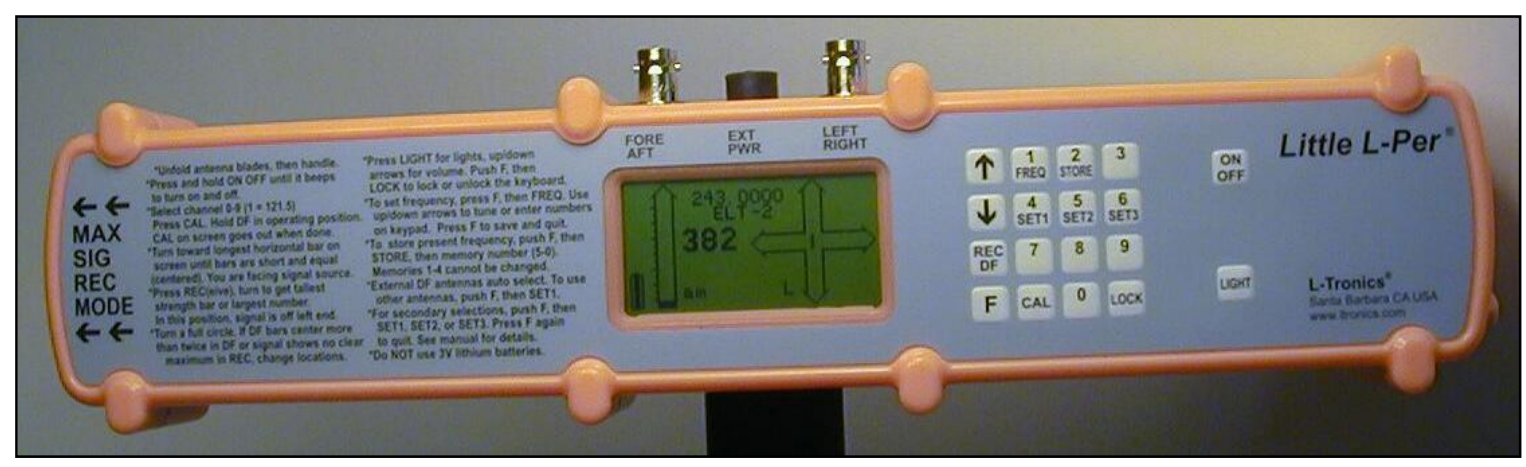

Figure 4: L-Tronics Little L-Per Portable Direction Finder[17] 


\subsubsection{Search and Rescue}

Outdoor Search and Rescue teams also use direction finding to locate missing people. The two primary types of transmitters used are Personal Locator Beacons (PLB) and Avalanche Beacons. PLBs are typically devices that use a GPS receiver and small transmitter to send a locating beacon to the satellite network in the event of an emergency. Once such device is the SPOT Satellite GPS Messenger which costs around $\$ 120$ and uses a $1.6 \mathrm{GHz}$ transmitter to send out the distress report [18]. Avalanche Beacons are different in that they are only used in very small deployment areas, and typically a mountaineering team will equip all its members with an Avalanche Beacon that is transmitting continuously during normal use. In the event of an emergency, members of the team that are not caught in the avalanche will switch use the built-in direction finding capabilities to locate the beacons from the lost persons. Avalanche beacons operate on the international standard frequency of $457 \mathrm{kHz}$ and typically cost around $\$ 200-500$. Figure 5 shows a typical avalanche beacon with a basic 5 LED DF system.

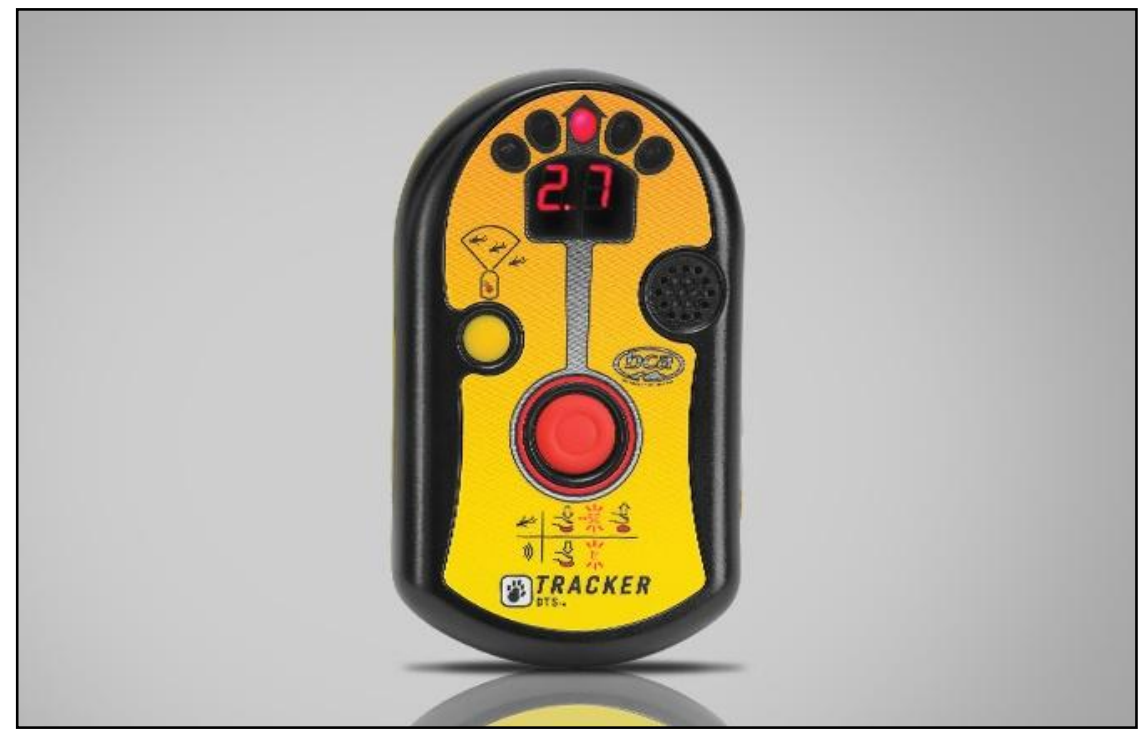

Figure 5: Backcountry Access Tracker DTS Avalanche Beacon [19] 


\subsubsection{Wildlife Tracking}

Researchers frequently use small radio transmitters for animal tracking to observe migration patterns and other behaviors. Beacons used for animal tracking are extremely diverse and cover a large range of sizes and transmitter output powers[20]. Figure 6 shows one example of such a small transmitter on a bee.

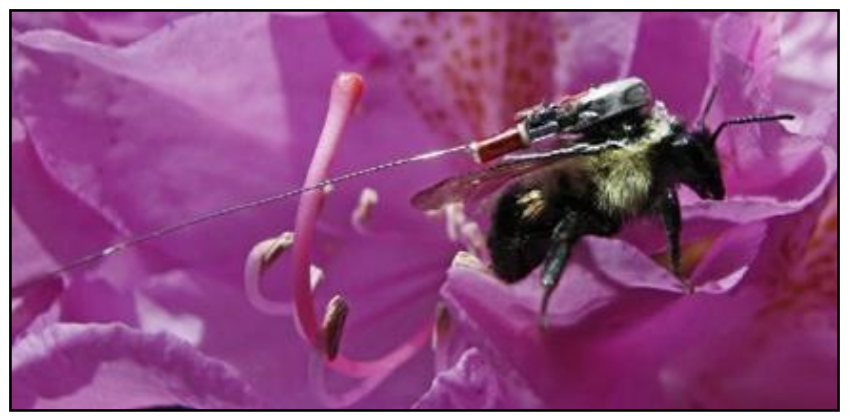

Figure 6: A 170 milligram radio tracking tag on a bee [21]

Typical radio frequencies for wildlife tracking are from $30-400 \mathrm{MHz}$ [20]. The VHF band is the most commonly used for these applications and typical receiver systems use a directional antenna (Figure 7) for manual signal-strength direction finding.

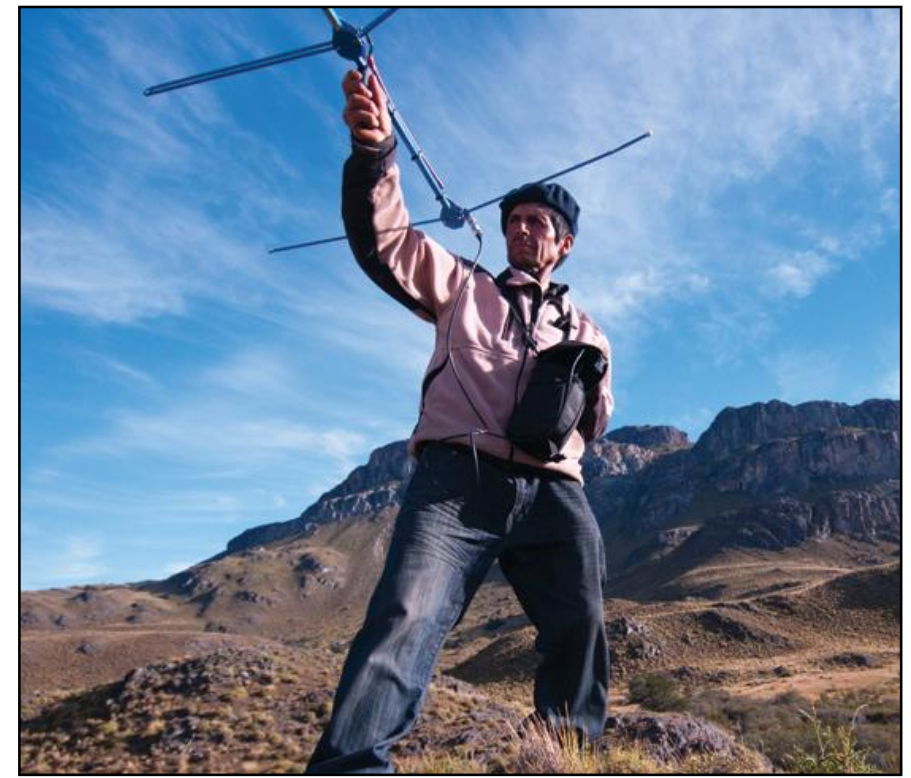

Figure 7: A manual VHF direction finding antenna system for wildlife tracking [22] 


\subsubsection{LoJack Vehicle Tracking}

LoJack is a private company that uses vehicle-mounted tracking beacons to help locate stolen cars. The stand-alone VHF transceivers typically remain in standby mode until activated by a satellite beacon when the vehicle is reported stolen. Detecting the car's signal is done through a number of quad-antenna pseudo-Doppler systems that are pre-installed in countless police vehicles around the country. When a beacon is detected by the head unit (Figure 8), a heading indicator shows the officer where the signal is coming from. The police officer can also call dispatch with the unique identifier code to get a vehicle description and further information to help in the search. LoJack Corporation reports a $90 \%$ recovery rate on cars, trucks, and SUVs [3]. The LoJack system requires satellites signals for activation and costly receivers in police vehicles for signal detection.

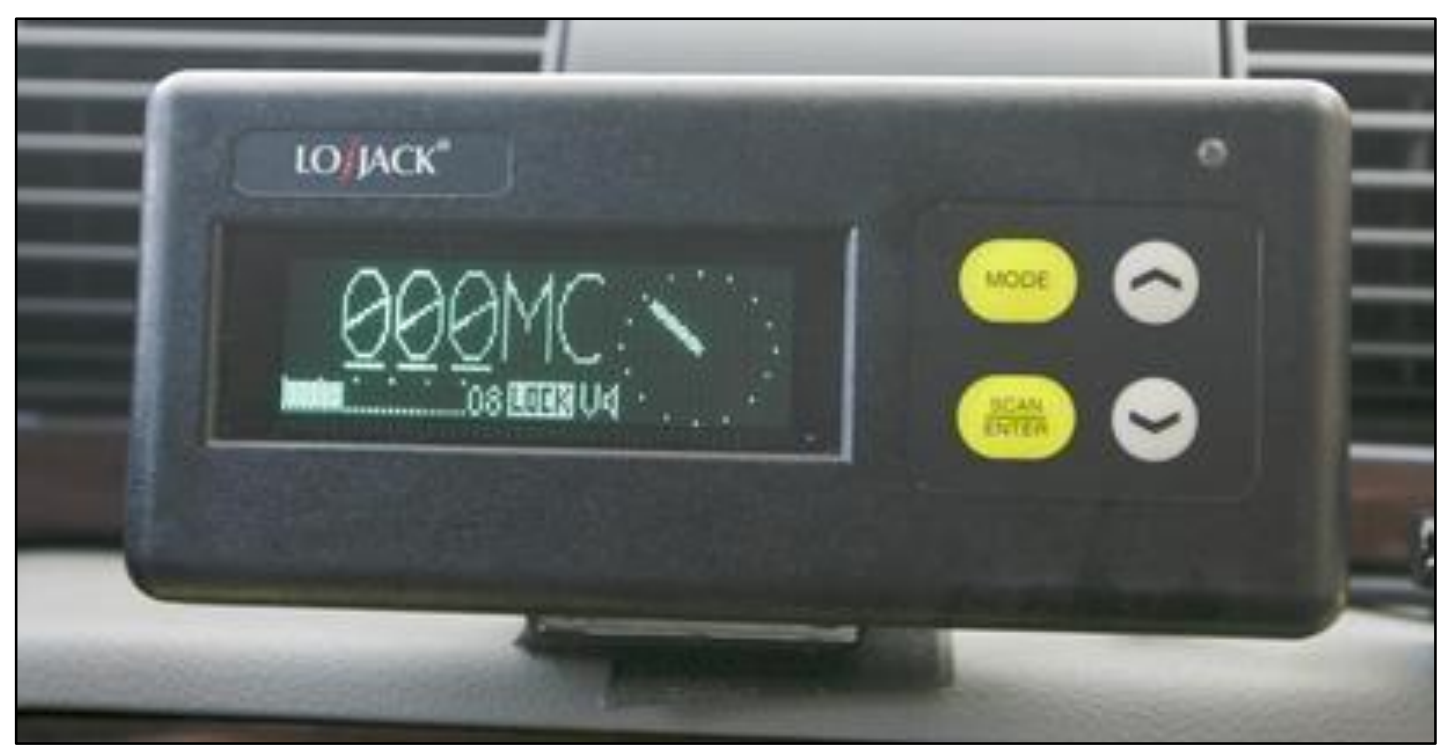

Figure 8: LoJack vehicle tracking control head unit [23] 


\subsubsection{Wireless E911 Phase 2}

Wireless E911 is a Federal Communications Commission (FCC) mandate from 2010 that requires cellular phone companies to provide location data for the origin of an emergency call to the appropriate 911 call center [24]. The initial phase of this mandate required that $95 \%$ of a provider's in-service phones must provide locating capabilities by December 31, 2005. E911 Phase II required that cell phone location could be determined to $50-300 \mathrm{~m}$ accuracy when requested by a Public Safety Answering Point (PSAP) [25]. Cellular providers use a variety of locating techniques including A-GPS (Assisted GPS), U-TDOA (Uplink Time Difference of Arrival), AOA (Angle of Arrival), and E-OTD (Enhanced Observed Time Difference). The most common of these is U-TDOA which measures the precise arrival of a cellular signal at various network base stations to determine the device's location. U-TDOA takes advantage of existing multi-billion dollar infrastructure of cell phone towers and vast computing backbone to provide both the sensing and processing required for accurate location reporting. TruePosition is an example of several companies that provide location services for cellular carriers. TruePosition uses several locating technologies including UTDOA, AOA, and a Hybrid Location Solution to develop a robust cellular locating network [26]. The primary disadvantage to these E911 systems is their dependence on elaborate existing hardware from the cellular industry. Cellular infrastructure is also unstable and prone to premature failure in disaster situations when systems become overloaded and power outages cause towers to go out of service [27]. Rural public service applications would require enormous installation costs to establish an equivalent infrastructure. 


\section{Problem Refinement and Scope}

The following list provides subjective metrics for each RDFN in order to characterize the existing systems and provide a basis for comparison between them.

- $\quad$ Rescue 21

○ Cost: HIGH, Complexity: HIGH

○ Network Implementation: Costal Networked Tower Sites

- RDF Method: 9-element Adcock Array, AOA [27]

- Disadvantages: Large, complex, and expensive.

- Aircraft ELT System

○ Cost: MED, Complexity: HIGH

○ Network Implementation: Satellite and Aircraft Monitoring

○ RDF Method: Satellite and manual ground team direction finding

○ Disadvantages: Satellite dependency, high failure rate

- Avalanche Beacons

○ Cost: LOW, Complexity: MED

○ Network Implementation: None.

- RDF Method: Manual ground team direction finding

○ Disadvantages: Requires skilled DF rescuer, small-area coverage

- Personal Locating Beacons

○ Cost: LOW, Complexity: MED/HIGH

○ Network Implementation: None.

○ RDF Method: GPS Position Reporting

○ Disadvantages: Satellite dependency and additional hardware needed

- Wildlife Tracking

○ Cost: LOW, Complexity: MED

○ Network Implementation: None.

- RDF Method: Manual ground team direction finding

○ Disadvantages: Requires skilled DF operator, small-area coverage

- LoJack

○ Cost: MED, Complexity: HIGH

○ Network Implementation: Satellite and vehicle based receivers

○ RDF Method: Pseudo-Doppler

○ Disadvantages: Satellite dependency and cost

- $\quad$ E911

○ Cost: HIGH, Complexity: HIGH

○ Network Implementation: Existing cell tower sites

○ RDF Method: U-TDOA, AOA, E-OTD

$\circ$ Disadvantages: High cost and dependence on infrastructure 
A thorough examination of all the existing RDF systems explored in 4.2 quickly reveals that all are either large-scale and high-cost (e.g. Rescue 21) or small-scale and very specialized implementations of an RDF system (e.g. wildlife tracking). The missing link between nationwide RDFNs and portable, handheld DF device's lies in a deployable RDFN that is available at a much lower cost than current networks and that has a much simpler architecture. Having a lost-cost, deployable VHF RDF network for public service and emergency response is the next step in enabling dozens of organizations to better manage their response and safety in an emergency.

This project focuses on finding an RDF solution that addresses the two major roadblocks of widespread RDFN use: cost and complexity. (Note that for the remainder of the document cost and complexity will be italicized when present.) For example, the total Rescue 21 system cost was estimated at $\$ 872$ million (increased from the initial acquisition cost in 1999 of $\$ 250$ million [28]) showing that large-scale RDFNs are extremely expensive. One of the primary objectives of this project is to minimize system cost to increase the availability of this technology to end users. The second major task is in system complexity both from an equipment and usability perspective. Existing RDF systems either have complex equipment requirements that greatly increase total cost (e.g. E911 and LoJack) or they have simple equipment but require highly skilled operators to locate them (e.g. wildlife tracking and avalanche beacons). In order to address these issues, the proposed system must maintain a simple hardware implementation and an accessible end user experience, while keeping expenses to a minimum.

Given the designated end user and additional system limitations, the following list of desired system characteristics is developed. 


\subsection{Proposed Design Feature List}

- Band of Operation: VHF

- Use a DF method to provide a minimum of $1 \mathrm{~km}$ locational accuracy

- APRS (Automatic Position Reporting System) network compatibility

- Same frequency reporting of signal metrics

- Maximize COTS usage for ease of repeatability

- Simple construction, installation, and operation

- Applicable to public service

- Ease of repairability

- Total per unit cost under $\$ 500$

- Manual target locating and data processing

- 1 week off-grid deployment capability

While this list provides for basic system functionality, in order to become a practical system implementation, a number of additional features are necessary. The following list of target features covers some of the important factors needed to make the system suitable for widespread use and long-term sustainability.

\subsection{System Comparison}

With the previous list of desired system features, the primary design decision was to choose a DF method that meets this feature set. Several different DF systems could be implemented, Table 1 shows two of these options.

\section{Table 1: Comparison of Proposed VHF RDF Network Technologies}

\begin{tabular}{|c|c|c|}
\hline DF Method & $\begin{array}{c}\text { Antenna } \\
\text { Requirements }\end{array}$ & Additional Required Components \\
\hline TDOA & Single Antenna & High-accuracy timing source \\
\hline Pseudo-Doppler & Quad-dipole array & Antenna switcher and analysis circuitry \\
\hline
\end{tabular}

Both of these primary DF methods have benefits and drawbacks for use in this particular system due to size and complexity limitations of each. 


\subsubsection{TDOA}

The TDOA system has the simplest hardware, but has a significant technical constraint requiring a highly accurate timing source for accurate direction finding. Another drawback of the TDOA system is that no directional information is given from the signal metrics. This requires at least 3 receivers to provide signal data in order for any locating to be successful.

\subsubsection{Pseudo-Doppler}

The pseudo-Doppler method does require a larger quad-antenna array [29], but provides directional information about the received signal which is advantageous when fewer receive sites are available. While the pseudo-Doppler system is not the simplest implementation, it is a widely-used technology in the amateur radio community. Therefore, technical support for a pseudo-Doppler system could be as easy as finding a local amateur radio club with some direction finding enthusiasts. There are several commercial Doppler systems available (Figure 9), but most are expensive or require significant technical skill to construct and use. With these challenges in mind, the first RDF prototype tested for this project was one using a pseudo-Doppler method for direction finding since it provides a directional heading from the receiver which can easily be used to determine signal source location and is of relatively simple construction.

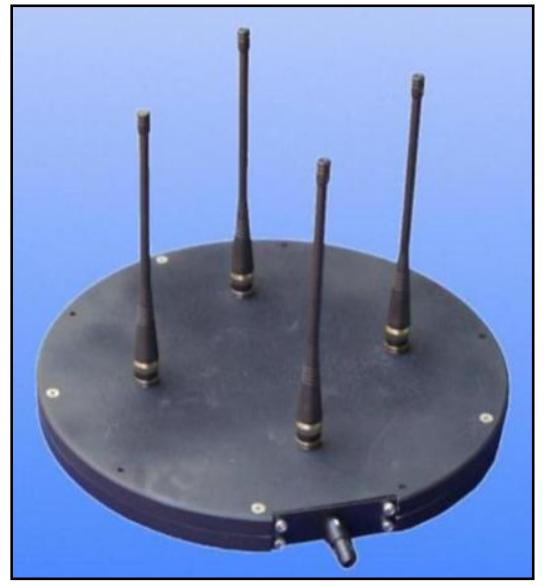

Figure 9: PicoDopp MiniMount Antenna Array [30] 


\section{Pseudo-Doppler Direction Finding Prototype}

The Pseudo-Doppler RDF system uses a phased antenna array to determine a signal's angle of arrival (AOA) and is based on the design from Mike Kossor WA2EBY shown in Figure 10[31]. The design had to be modified to achieve the desired features and make use of COTS components, simplify construction, and include a microprocessor controlled antenna switching circuit to modernize the design and improve system capabilities. For a complete RDF network implementation, the antenna system would need to be constructed and integrated with a radio transceiver and data interface to link to the APRS network.

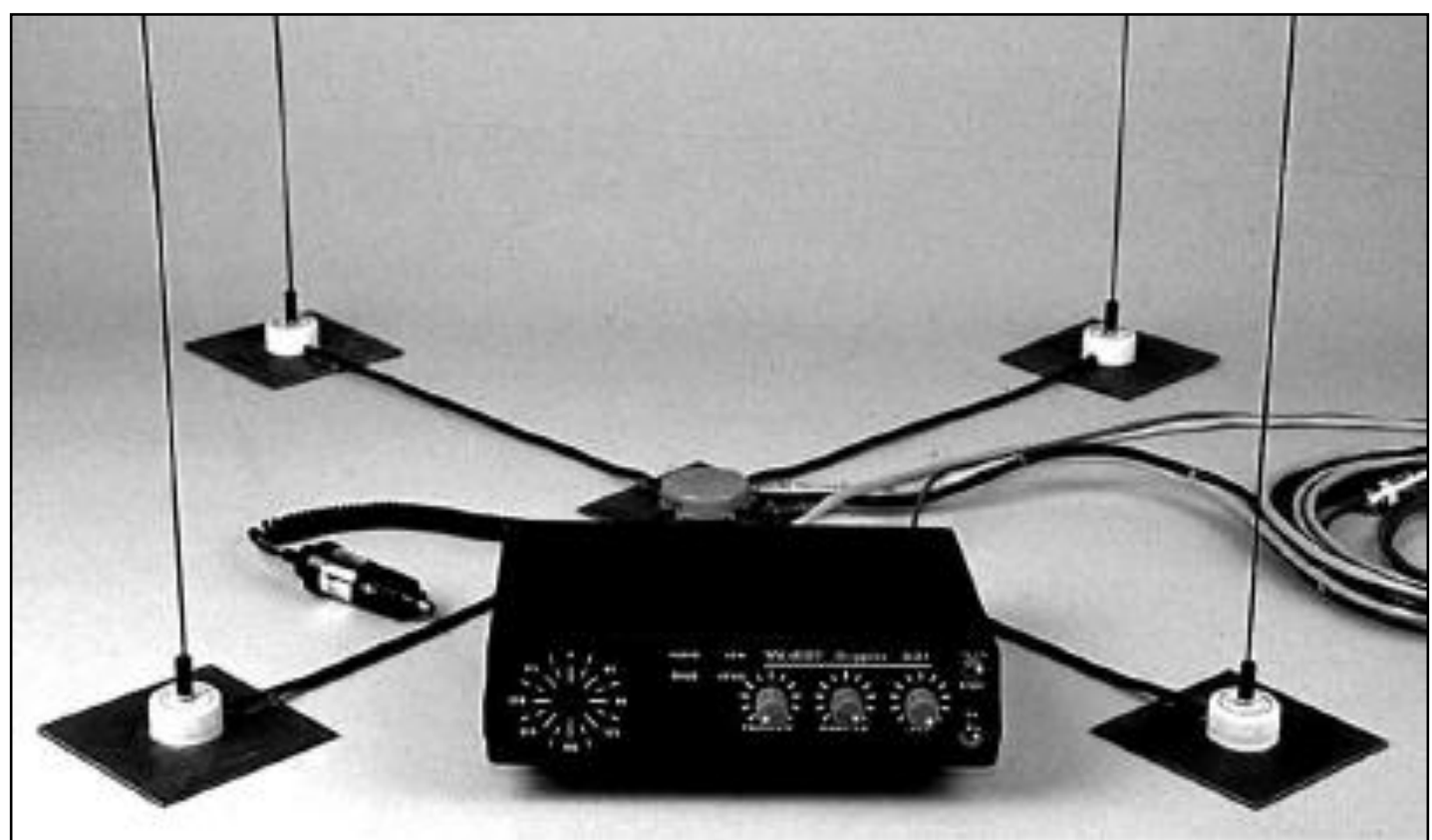

Figure 10: A pseudo-Doppler RDF system by Mike Kossor WA2EBY [31] 


\subsection{Pseudo-Doppler Direction Finding Theory of Operation}

A pseudo-Doppler direction finder uses the Doppler effect to determine a signal's angle of arrival (AOA) at the receive site. If an antenna is moving towards a signal source, the perceived receive frequency is slightly higher than if it was stationary. Conversely, as the antenna moves away from the source, the perceived frequency is lower[31]. By rotating an antenna in a circle, continually increasing and decreasing the distance to the source, the frequency becomes modulated at the same frequency as the speed of rotation. This principle is shown in Figure 11 below.

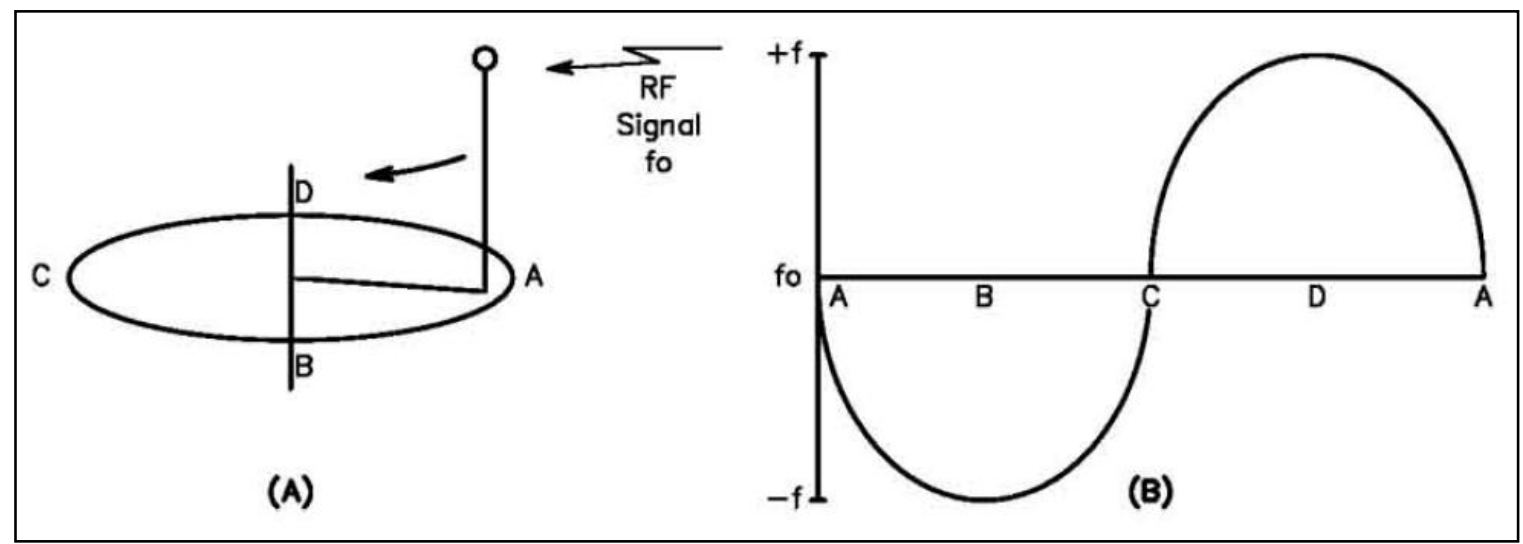

Figure 11: (A) A rotating monopole antenna and (B) the resultant Doppler frequency modulation[31]

The rate at which the antenna rotates is based on the following formula which gives the peak frequency shift ( $\Delta f$ in $\mathrm{Hz}$ ) in terms of antenna rotational radius ( $r$ in meters), angular velocity of rotation ( $\omega$ in radians per second), speed of light ( $c$ in meters per second), and the carrier frequency of the received signal $\left(f_{c}\right.$ in $\left.\mathrm{Hz}\right)$ [32] [33].

$$
\Delta f=\frac{r \times \omega \times f_{c}}{c}
$$

This equation is then evaluated for an incoming signal at $144 \mathrm{MHz}$, with a 19 inch antenna rotation $(48.26 \mathrm{~cm})$, and a $500 \mathrm{~Hz}$ desired Doppler signal tone. 


$$
\begin{aligned}
& \omega=\frac{\Delta f \times c}{r \times f_{c}}=\frac{(500 \mathrm{~Hz})\left(3 \times 10^{8} \mathrm{~m} / \mathrm{s}\right)}{(0.4826 \mathrm{~m})(144,000,000 \mathrm{~Hz})}=2158.45 \mathrm{rad} / \mathrm{s} \\
& r p m=2158.45 \mathrm{rad} / \mathrm{s} \times \frac{60 \mathrm{~s}}{1 \mathrm{~min}} \times \frac{1 \text { rotation }}{2 \pi \text { radians }}=20,612 \mathrm{rpm}
\end{aligned}
$$

\subsubsection{Mechanical vs. Electronic Rotation}

The result above shows that at VHF frequencies, the antenna needs to rotate at tens of thousands of RPM to produce a usable Doppler signal of a few hundred hertz within the audio frequency range of the data radio. The mechanical implementation of such a system is impractical and involves complex and high-maintenance systems to support and rotate a VHF-sized antenna. This complex mechanical solution can be realized by using an electronically switched array of antennas. This can be done a number of ways including through a rotating capacitive switch as used in the HFDF systems [9] or diode-biased switching as will be used in this design. Further analysis of diode switching is described in section 6.3.1 Antenna Switcher Design and Construction.

By arranging a number of antennas in a circular pattern and electronically switching around the ring of elements, the effect of spinning a single element at high-speeds is mimicked without any physical motion of the elements. Designs have been built at high frequency with dozens of elements in the array, but for a VHF system the effectiveness of a 4-antenna array is sufficient for directional accuracy [32] and provides a reasonable sized array for this project's specifications ( $\sim 19$ inches per side of the antenna array). 


\subsection{System Overview}

The basic pseudo-Doppler system requires a 4-element antenna array that is driven by an antenna switcher to electronically rotate through the elements. The resulting output RF signal output is then routed to an FM receiver that demodulates the signal and passes the resulting audio signal to the signal processing circuit. The signal processor uses the synchronized control signals from the antenna along with the received audio from the FM receiver to determine the signal direction. The final step to complete the network is to transmit the signal metrics back to the centralized receive station for data processing. Figure 12 shows the system block diagram of this configuration.

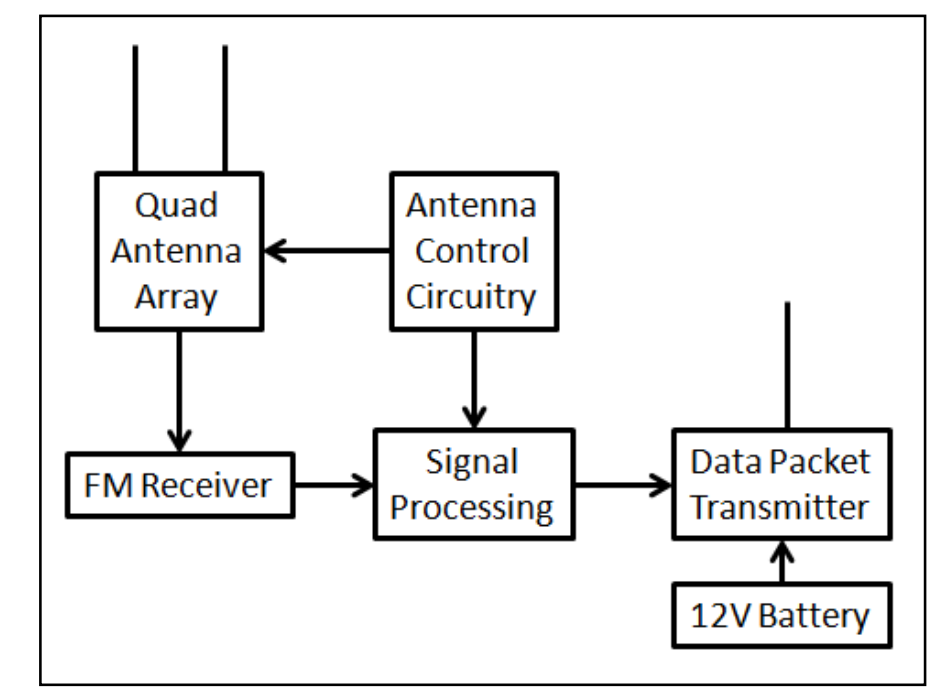

Figure 12: Pseudo-Doppler RDF system block diagram

\subsubsection{Equipment List}

- Antenna Switcher Component costs (approximately $\$ 40$ )

- Tram 1100 1/4 wavelength VHF magmount antenna (qty 4 at \$20/each) [34]

- Arduino UNO Microcontroller (\$29.95) [35]

- Argent Data Systems Tracker 3 APRS Tracker (\$95) [36]

- Friendcom FC-301/D 5 watt VHF Data Transceiver (\$149) [37]

- VHF Whip Antenna (\$9)[38]

- 12V 35Ah Werker Deep Cycle AGM Battery (\$87)[39]

- Anderson Powerpole connectors and battery wire (\$10)

\section{Total system cost: $\$ 500$}




\subsection{System Design Modification and Construction}

\subsubsection{Antenna Switcher Design and Construction}

The basic system construction for this prototype was based on the schematics developed by Mike Kossor WA2EBY (Figure 13) with some modification. Some designs include antennas built using metal welding rods, but simplifying the design was a primary goal so low-cost COTS antennas were used instead. The antennas chosen were the Tram $11001 / 4$ wavelength VHF magmount antenna available from Ham Radio Outlet for \$20 [34]. The antenna switching design by Kossor uses blocking diodes at the base of each antenna which would require modification to the magmount to include D8/9/10/11 and L2/3/4/5 (Figure 13). In order to utilize COTS antennas for this design and minimize complexity, these 4 diodes were removed from the design as shown in Figure 14. Analysis in section 6.3.1.2 Diode Characterization shows the performance results of this design change.

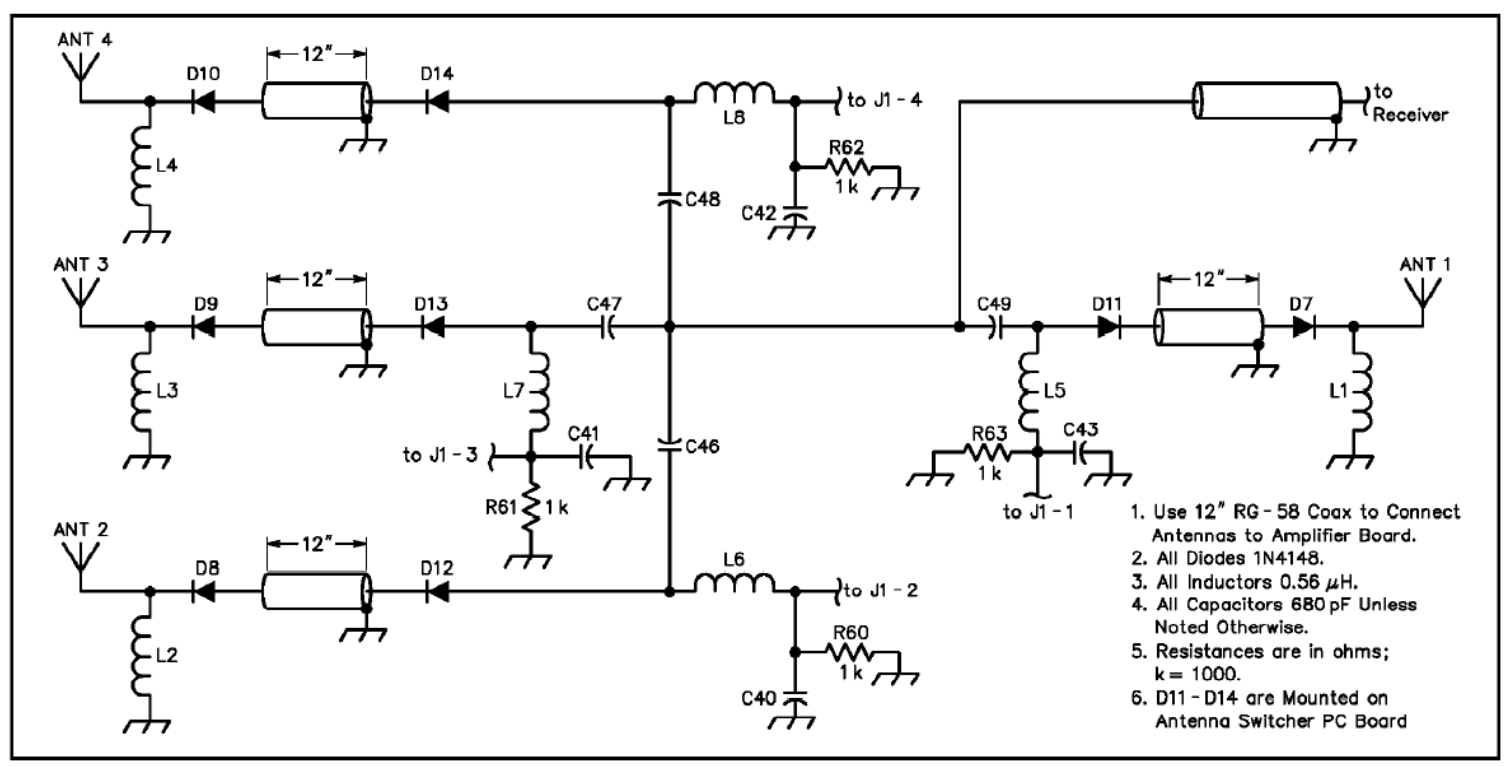

Figure 13: Antenna switcher schematic [29] 


\subsubsection{Antenna Switcher Schematic}

The simplified antenna switching schematics is shown in Figure 14. The 4 inductors $\mathrm{L} 2 / 3 / 4 / 5$ are still required to provide the DC switching bias ground and were moved to the other side of the coax next to D11/12/13/14 to avoid any modification to the COTS antenna base. For Antenna 1, the diode biasing is provided from Control1, through RFC's L2 and L1. The $0.56 \mu \mathrm{H}$ SMD inductors used in this project had an isolation at VHF of $-19 \mathrm{~dB}$ at $147 \mathrm{Mhz}$ which provides an acceptable RF choke for this portion of the circuit. C6 helps reduce any leakage RF from travelling along the control line to the switching control from the Arduino. C1 is a DC blocking capacitor for the output coupling to the FM receiver output.

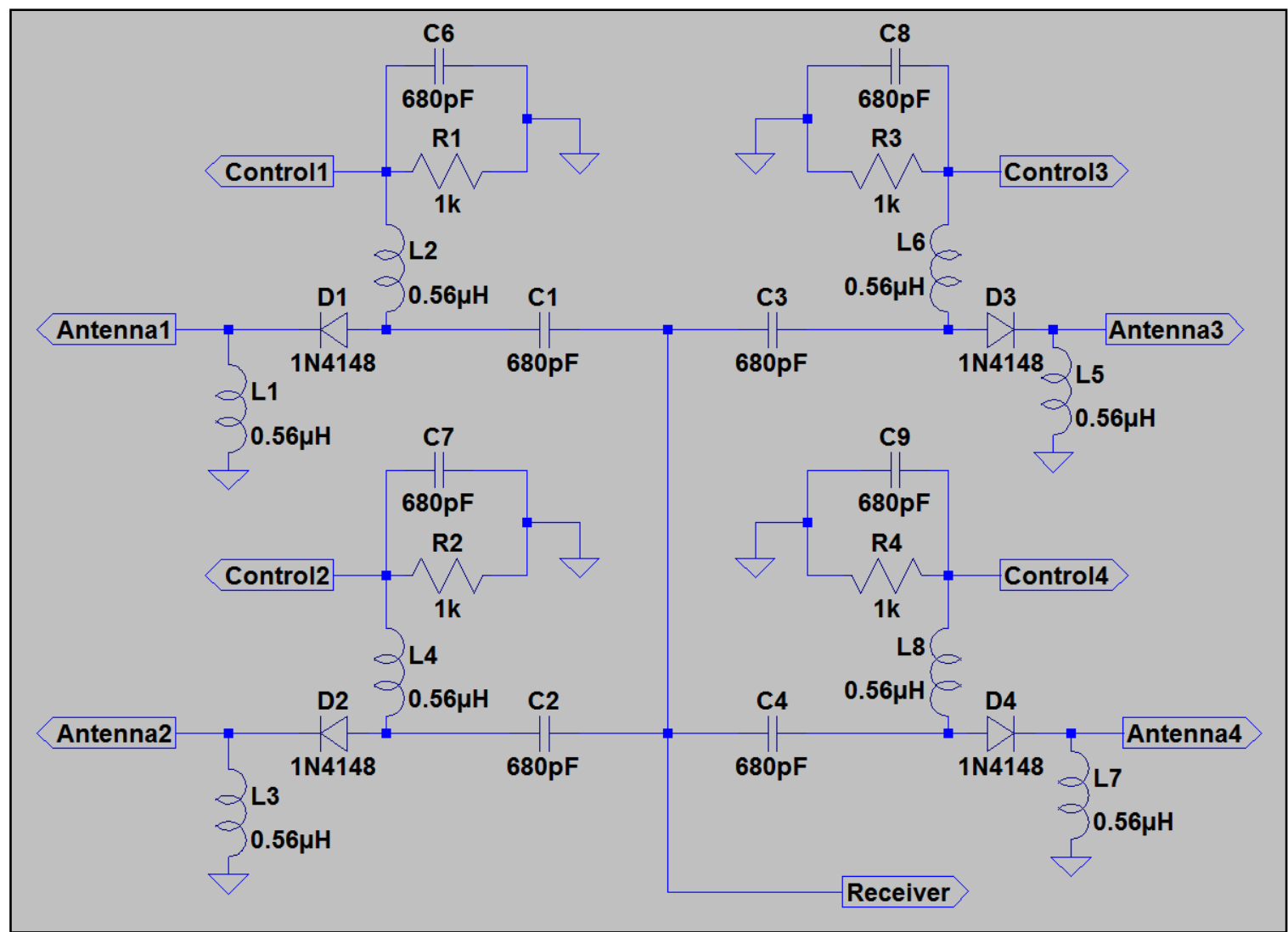

Figure 14: Improved antenna switcher schematic 


\subsubsection{Diode Characterization}

The primary limitation of removing one set of diodes from the switching circuitry is that the antenna isolation is reduced. Per the testing done by Kosser, the two types of 1N4148 through-hole diodes he tested had approximately $25.25 \mathrm{~dB}$ and $23.55 \mathrm{~dB}$ insertion loss for the 333-1N4148 and 583-1N4148 respectively [29]. Figure 15 shows the SMD 1N4148 test results indicating an insertion loss of $21.21 \mathrm{~dB}$ at $145 \mathrm{MHz}$. This test was done on the nritsu MS4622B Vector Network Measurement System using a small stripline test fixture to mount the diode upon. Kosser also performed insertion loss and isolation testing on various diodes to validate his design choice to use the 1N4148 diode for the antenna switching [29].

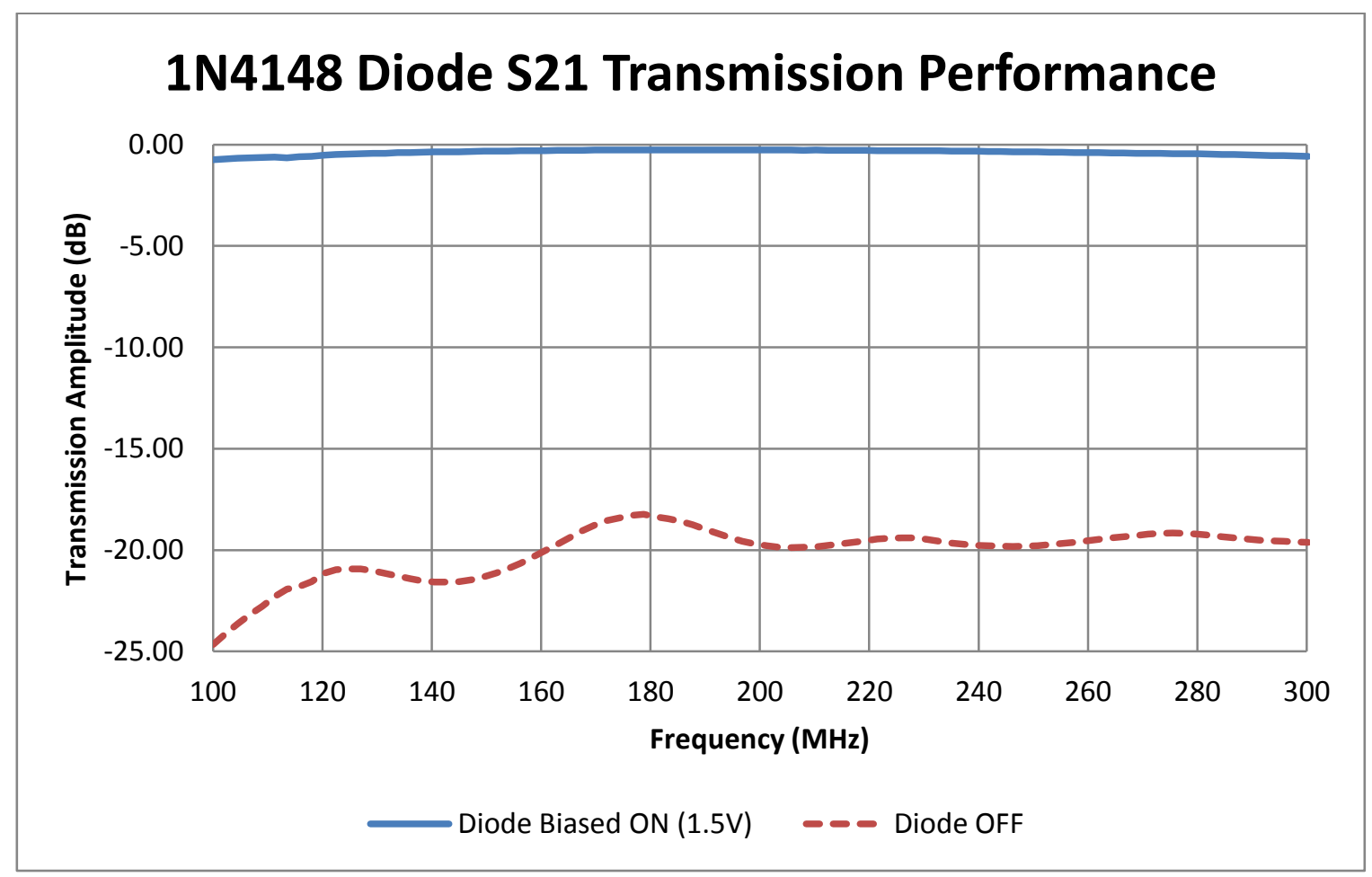

Figure 15: Graph of 1N4148 diode isolation characteristics under biased and nonbiased conditions 


\subsubsection{Antenna Switcher Board Layout}

The antenna switching board was built on copper-clad circuit board using a Dremel tool to cut around the traces. The bare PCB is shown in Figure 16. The four "islands" near each antenna input are where the switching control inputs bias the switching diodes. Figure 17 shows the populated PCB with each of the 4 antenna inputs (top-1, right-2, bottom-3, left-4). The additional connector on the top left is for the RF output to the FM receiver. The antenna switcher was fully characterized from $10 \mathrm{MHz}-3 \mathrm{GHz}$ and is shown in Appendix D: Antenna Switcher Characterization.

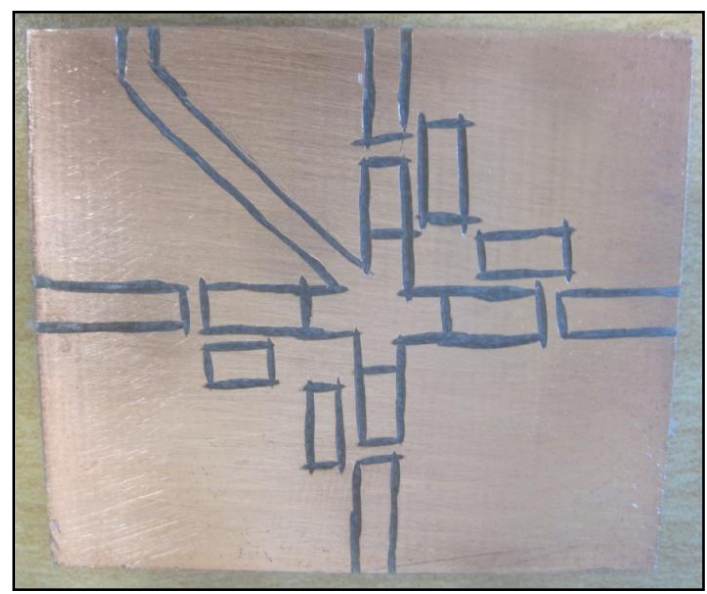

Figure 16: Antenna switcher PCB with etched traces

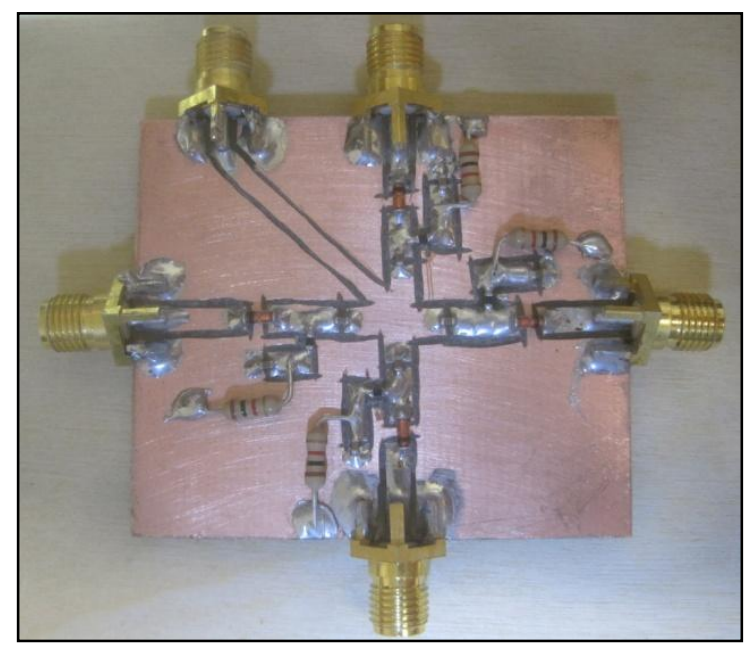

Figure 17: Populated antenna switcher PCB 
The completed antenna switching board with COTS magmounts and switching control lines is shown in Figure 18 below. The multicolor antenna control cable connects at each of the biasing islands. The FM receiver output is shown on the top of the board via the smaller SMA cable. It is worth noting that the board's layout is a physical analog to the electrical switching that is performed by the diodes.

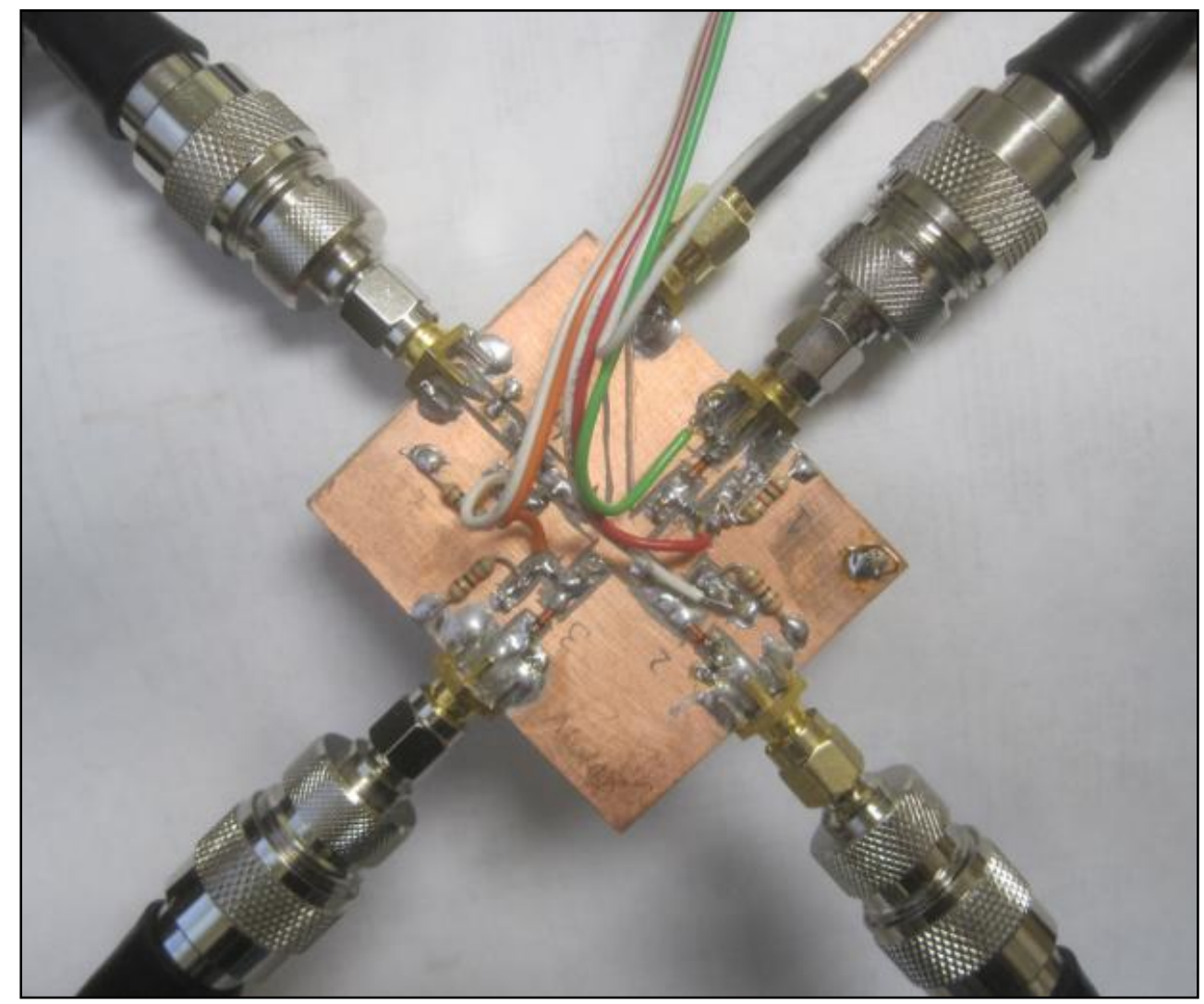

Figure 18: Completed antenna switching board (using the Improved antenna switcher schematic from Figure 14) 


\subsubsection{Antenna Switcher Operation}

Figure 19 shows a detailed image of the antenna 1 biasing circuitry. The orange 1N4148 diode is to the right of the upper SMA connector and is followed by the DC-blocking capacitor. The two black SMD inductors are shown leading to the red bias control cable and the green grounding cable.

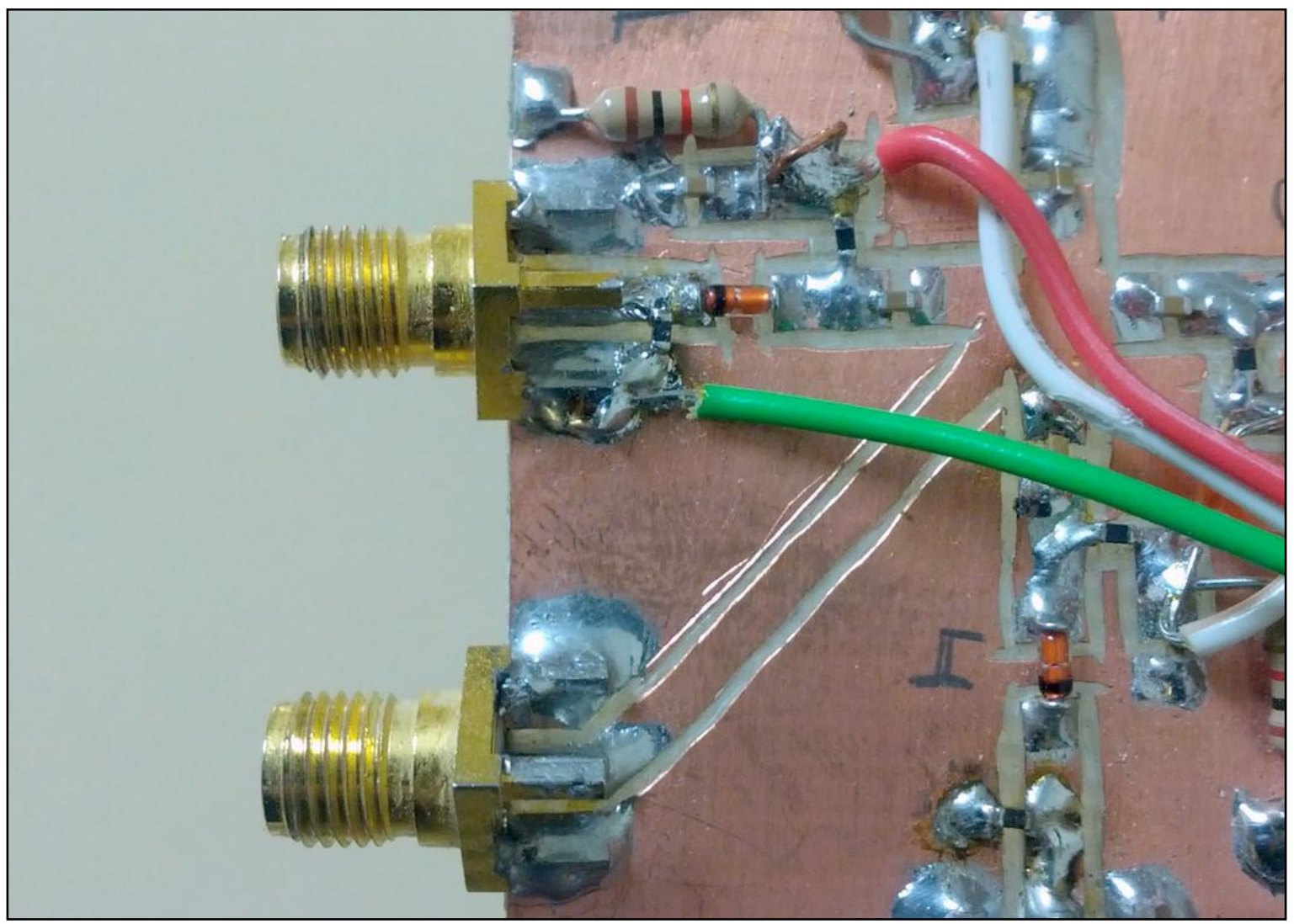

Figure 19: Close-up of Antenna Port 1 and Fm Receiver Port of the Antenna Switcher 
Figure 20 shows the DC biasing of the 1N4148 that brings the diode into conduction. Figure 21 shows the RF path in the forward-biased condition for the received signal to travel from the selected antenna to the FM receiver port.

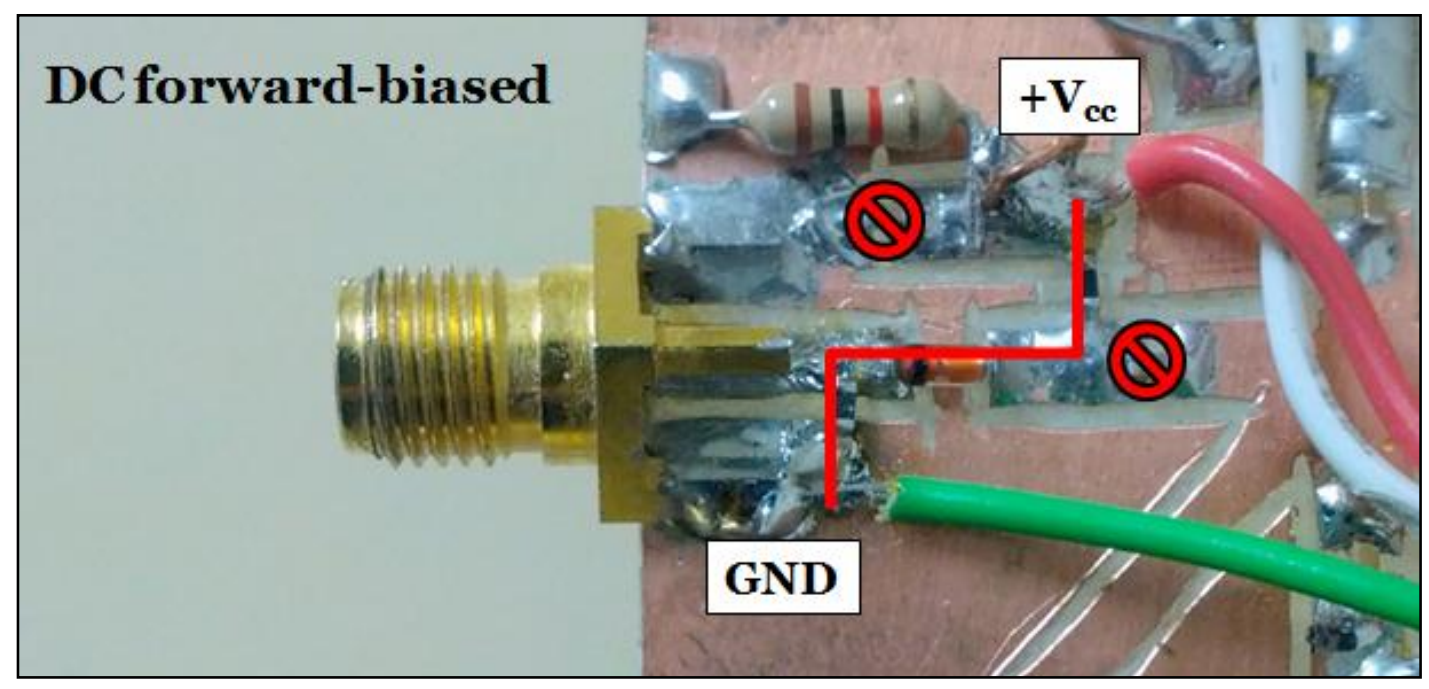

Figure 20: Antenna $1 \mathrm{DC}$ forward-biased detail diagram

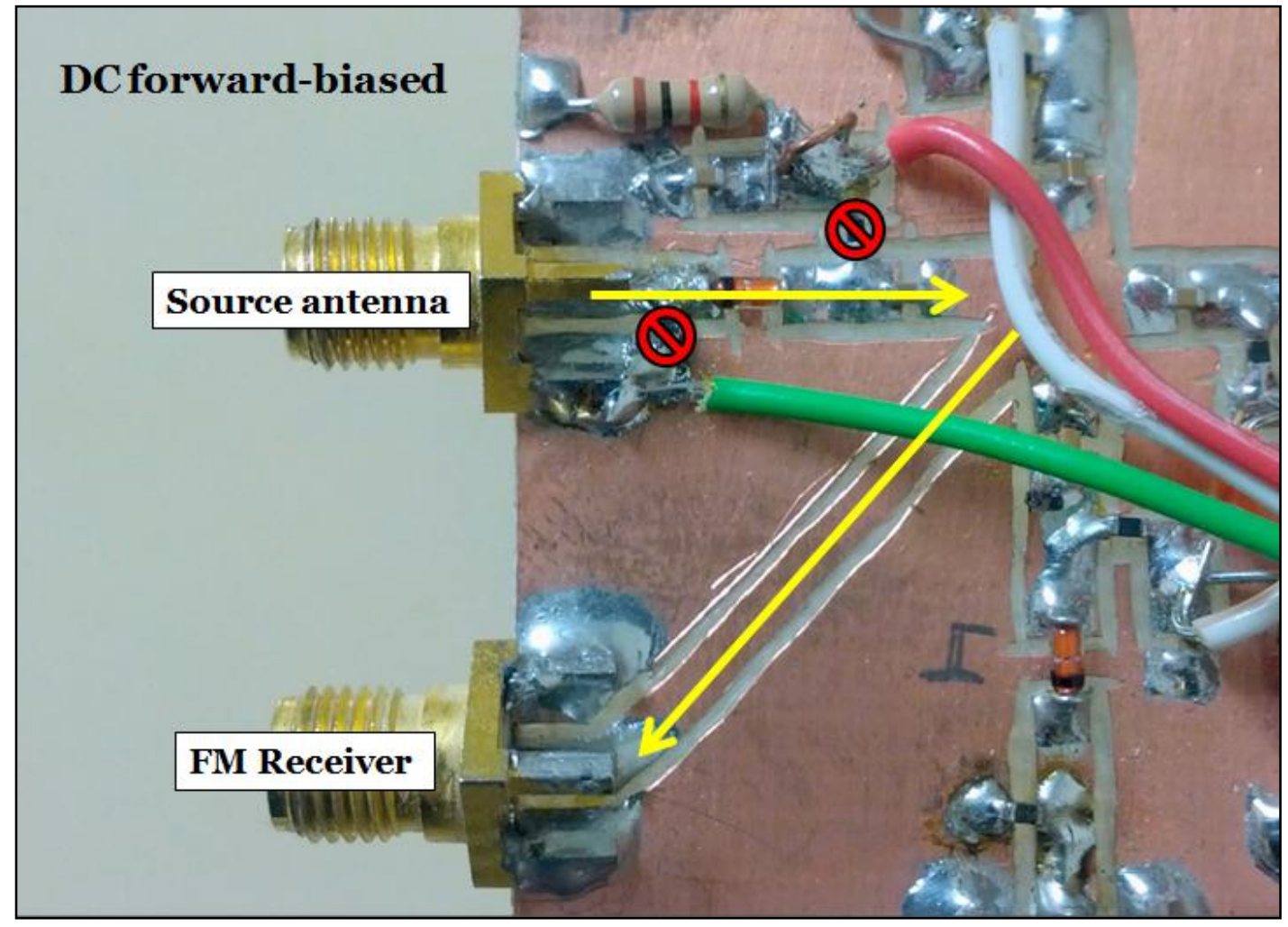

Figure 21: Antenna 1 DC forward-biased RF path diagram 
Figure 22 shows the non-biased condition where the diode is not-conducting and therefore blocks the RF path from the antenna.

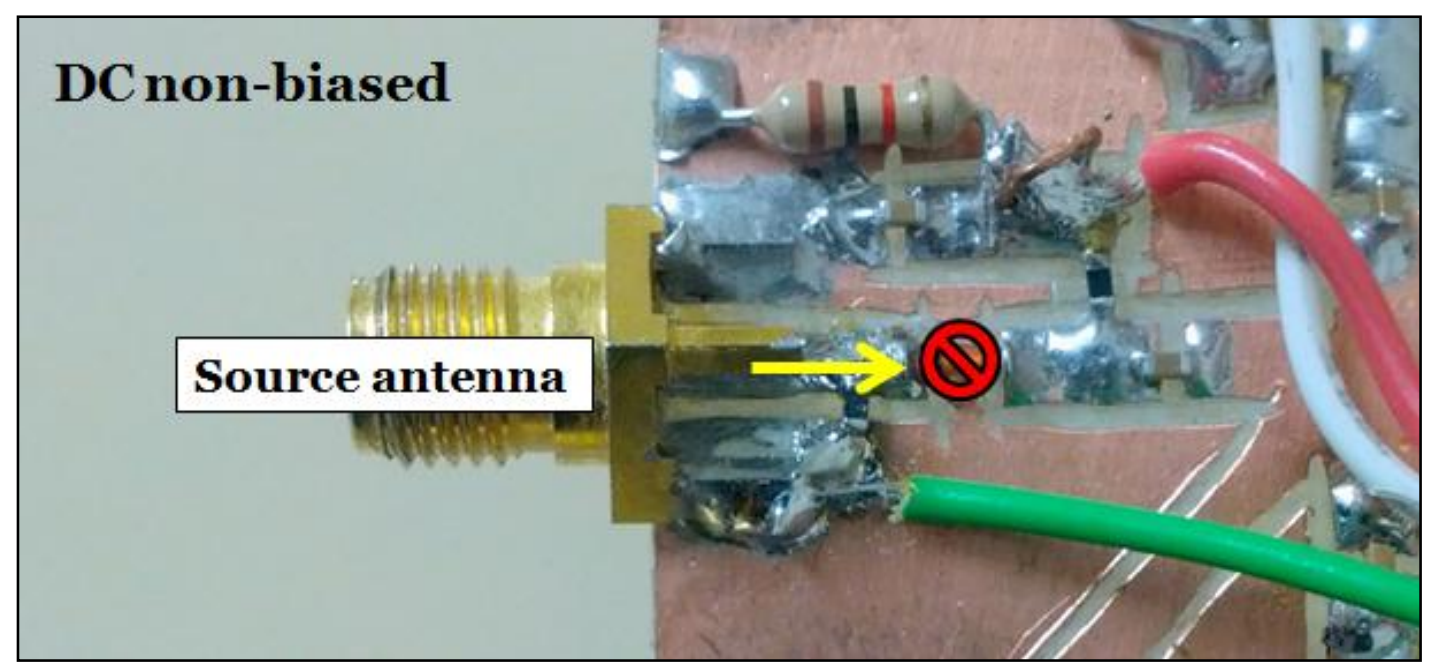

Figure 22: Antenna 1 non-biased antenna isolation condition 


\subsubsection{Antenna Switching Control}

The original design by Kossor calls for a discrete-component antenna switching control shown in Figure 23. This circuit was built and tested for verification and is shown in Figure 24. The design was simplified to just the switching control elements for testing.

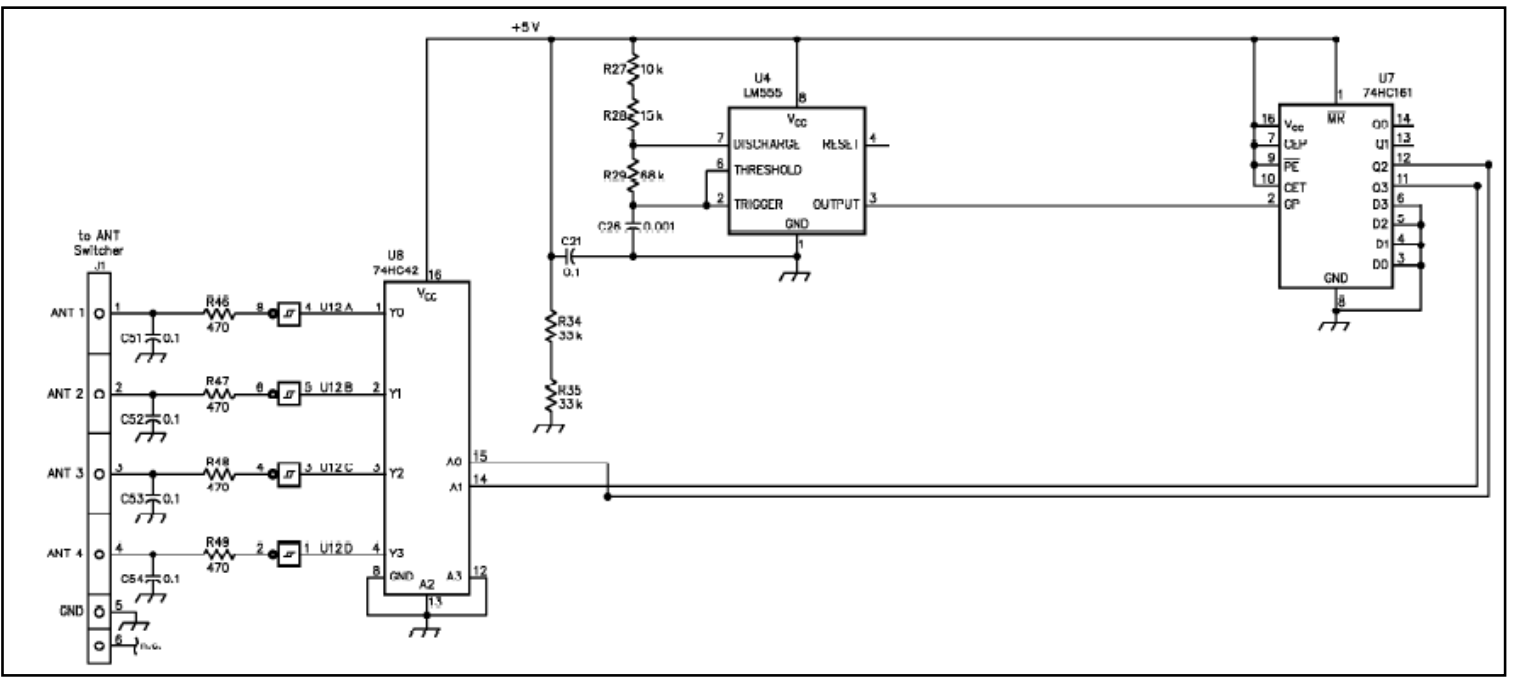

Figure 23: Simplified antenna switching control circuit schematic [29]

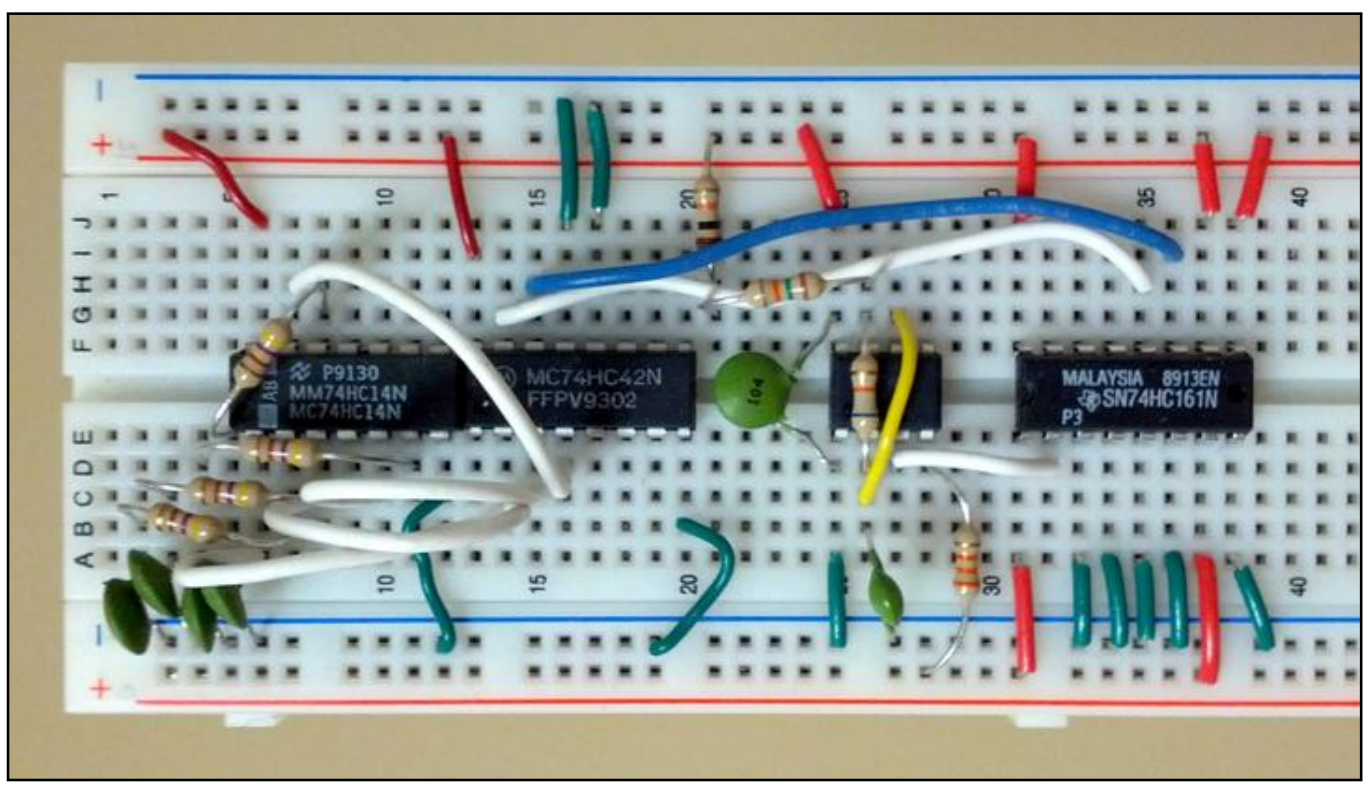

Figure 24: Discrete component antenna switching control circuit 
The discrete component antenna switching control circuit was tested to have a reference circuit use for comparison. Figure 25 shows the output signals from control signals 1 and 2 . The settling time of each signal is noteworthy since it softens the control signal and might affect the antenna switching performance. Also notable is the switching frequency which is $586.9 \mathrm{~Hz}$ and the peak to peak voltage of the control signals of $6.5-6.8 \mathrm{~V}_{\mathrm{pp}}$. It should be noted that the output voltage for this circuit was designed to drive 2 diodes in series. Since the simplified antenna switcher design only uses single diode biasing, the signal levels can be reduced.

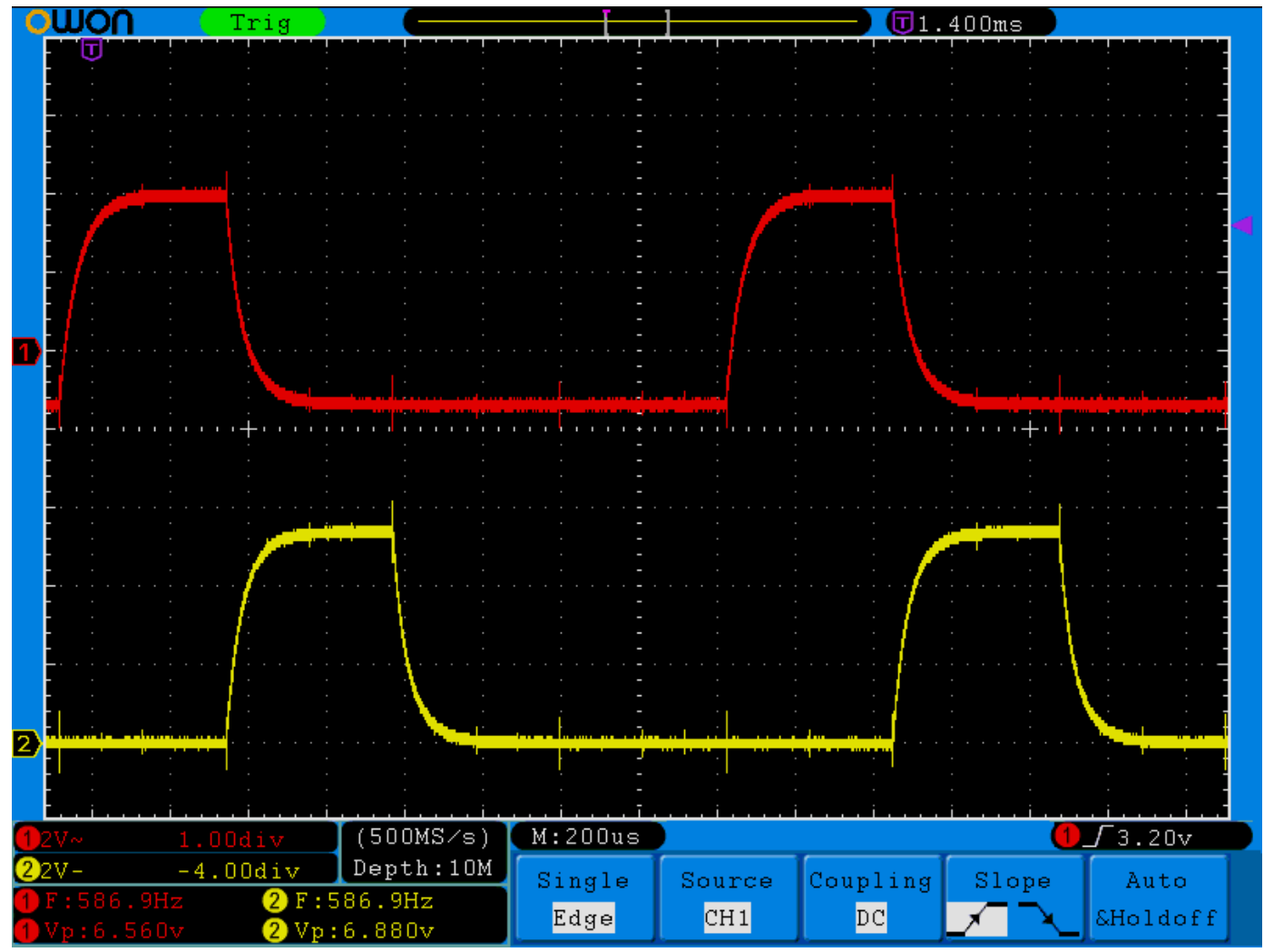

Figure 25: Discrete component antenna switching control signals 
After the antenna control circuit was tested and functional, the control circuit was completely replaced with an Arduino UNO (ATMega328P) to improve system flexibility and, most importantly, reduce complexity. Since the Arduino is a commonly used microcontroller and is simple to program, it is a good component to use for this design. The Arduino provides the time-synced antenna switching signals as well as the $500 \mathrm{~Hz}$ reference signal for the signal processor. Figure 26 shows the 4 antenna switching signals being generated by the Arduino. Note that the 4 antenna control signals were overlaid in this image for clarity. Channel 1 is the reference channel and each progressive signal is shown with its correct time delay so that only one antenna is on at a given time.

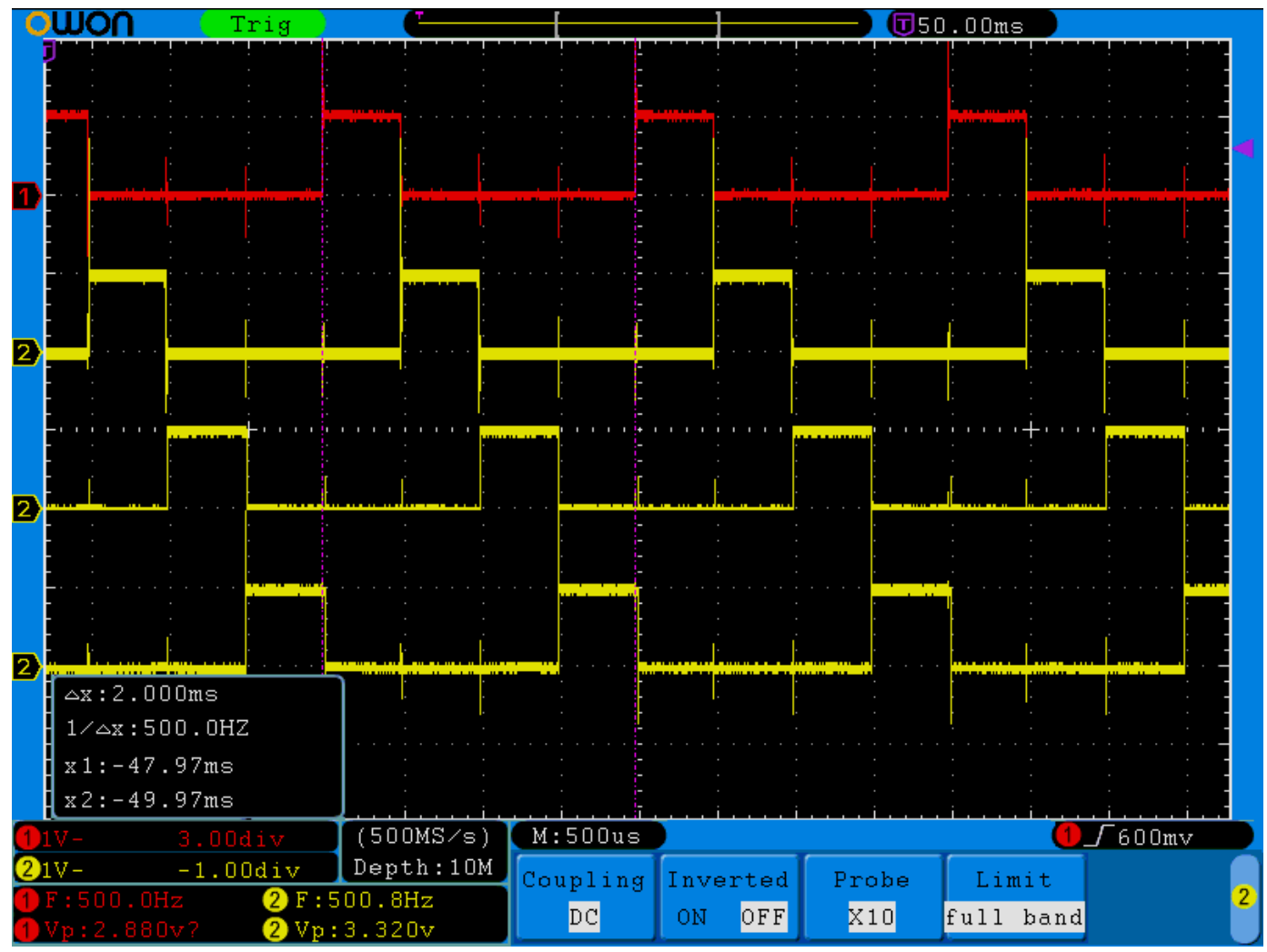

Figure 26: Arduino generated antenna switching control signals

The Arduino-based control circuit is able to provide a much more stable frequency reference of $500 \mathrm{~Hz}$ with a control voltage of $2.8-3.2 \mathrm{~V}_{\mathrm{pp}}$. 
Figure 27 shows the Arduino based control circuit implementation initially being tested with 2 antennas. The Arduino code for this controller is shown in Appendix A: Arduino Antenna Switcher Code. The code for the antenna switching was written in such a way to provide consistent timing for each antenna transition and minimize any errors in the way each antenna was selected.

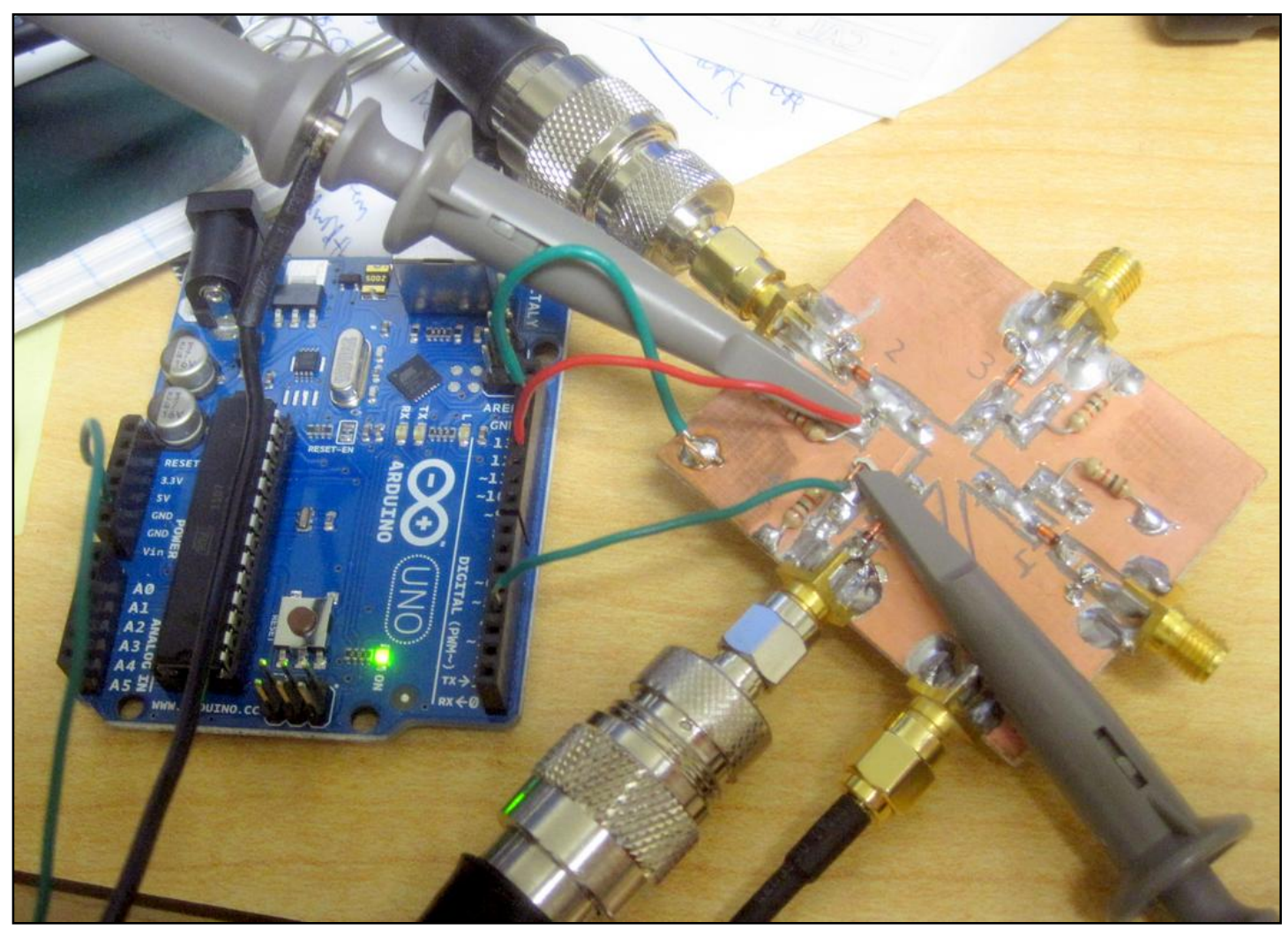

Figure 27: Arduino UNO controller antenna switching tests 
Using this control circuitry to drive the antenna switcher with the RF output going into the antenna port of a Yaesu VX-5R FM transceiver, the $500 \mathrm{~Hz}$ Doppler tone was observed on the audio output from the radio when receiving an FM signal on the radio. An FFT of the audio signal from the VX-5R shows the produced Doppler tone from the Doppler system along with the expected odd-harmonics generated by the square wave control signals. The subsequent harmonics are filtered out in DSP leaving only the $500 \mathrm{~Hz}$ fundamental frequency to use for the Doppler analysis.

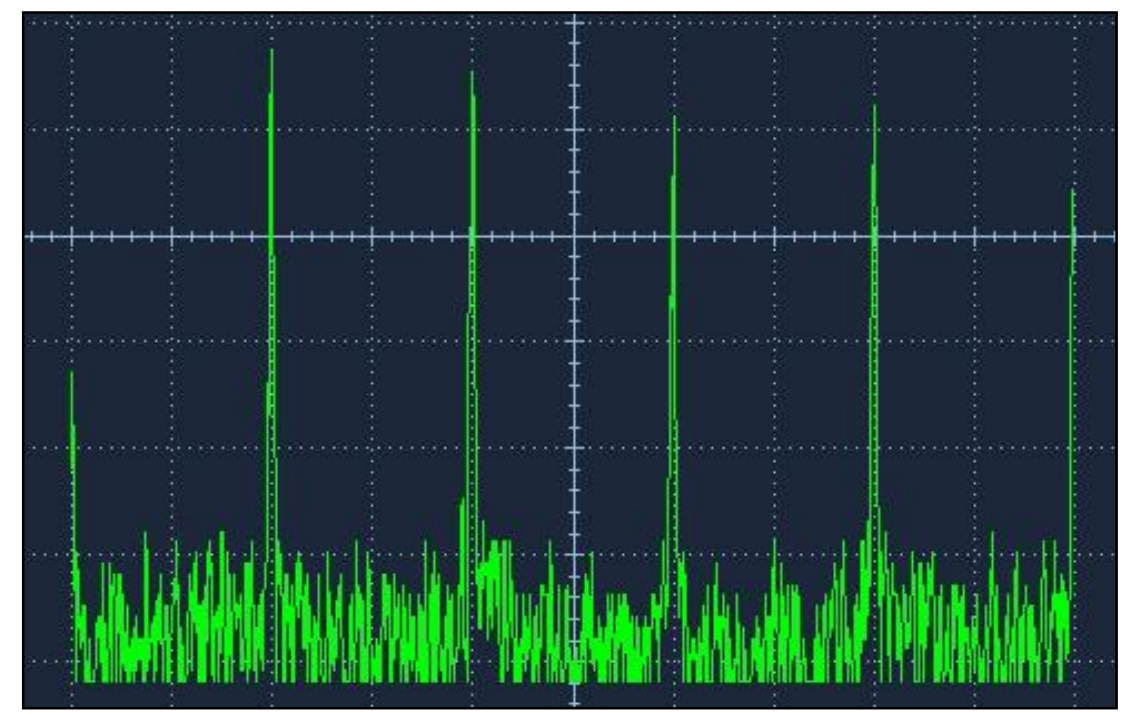

Figure 28: VX-5R demodulated FM audio output FFT (250Hz per horizontal division) 


\subsection{Pseudo-Doppler RDF System Setup and Testing}

In the completed receiver system, signal processing would be implemented in the Arduino or a similar microcontroller to keep the system compact and simple. For the purposes of testing the pseudo-Doppler RDF receiver, the SoundDoppler V1.20 software developed by Lodewijk Baars PA3BNX was used to perform the signal analysis [40] [41]. This software implements the filtering and frequency comparison of the Doppler system using DSP and provides a simple visual output display of the measured direction heading of the received RF signal. Very simple controls are also provided to calibrate the system and synchronize the switching and audio signals. This program requires the switching reference frequency to be input on the left channel of the computer's soundcard input and the demodulated FM audio from the receiver on the right channel. This was easily accomplished using a simple audio adapter board developed by Justin Kenny KJ6KST [42] to patch the Arduino reference frequency output and the audio output from the Yaesu VX-5R (and FT-60R) used in these tests. The netbook computer used for this test does not include a line-in port so a $30 \mathrm{~dB}$ audio pad previously built by the author was required to bring the line-level audio to mic-levels for the software to use and prevent overdriving the computer's audio input circuitry. The complete test system consisted of: 4 magmount antennas, antenna switcher PCB, Arduino UNO, 30dB line-level adapter, audio patch board, FM radio transceiver, and netbook computer as shown in Figure 29. 


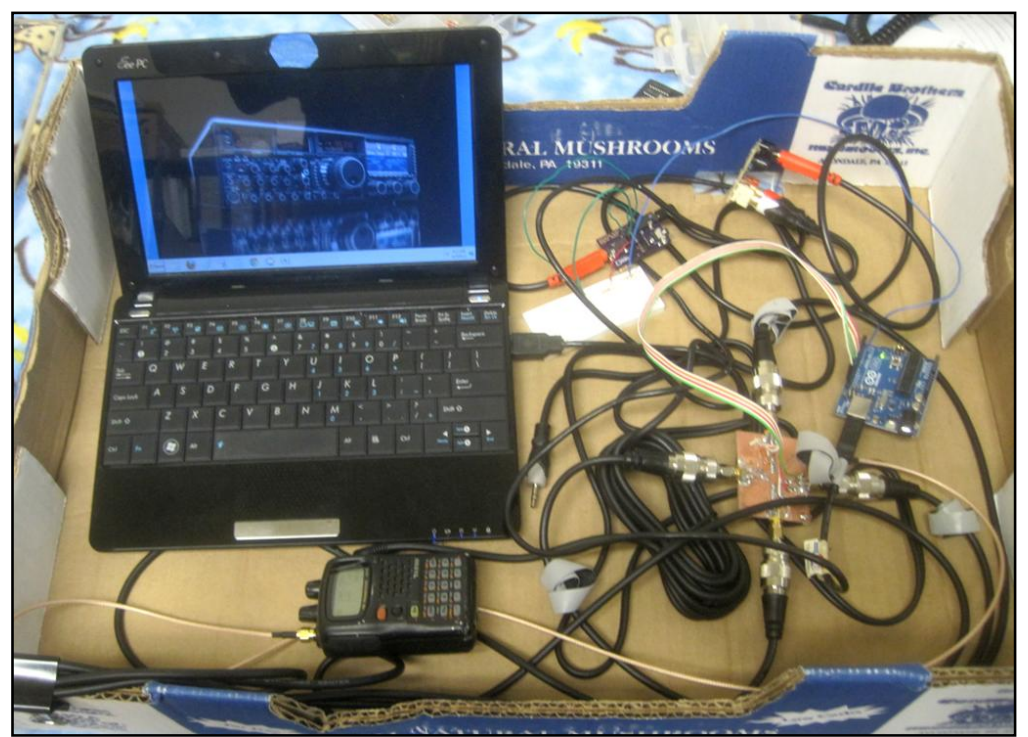

Figure 29: Pseudo-Doppler system test equipment configuration

The complete test system was installed into a mobile testing platform in Figure 30 and tested near San Luis Obispo, CA. The additional antenna on the rear was used for test coordination and was placed out of the Doppler array plane to minimize parasitic element interference.

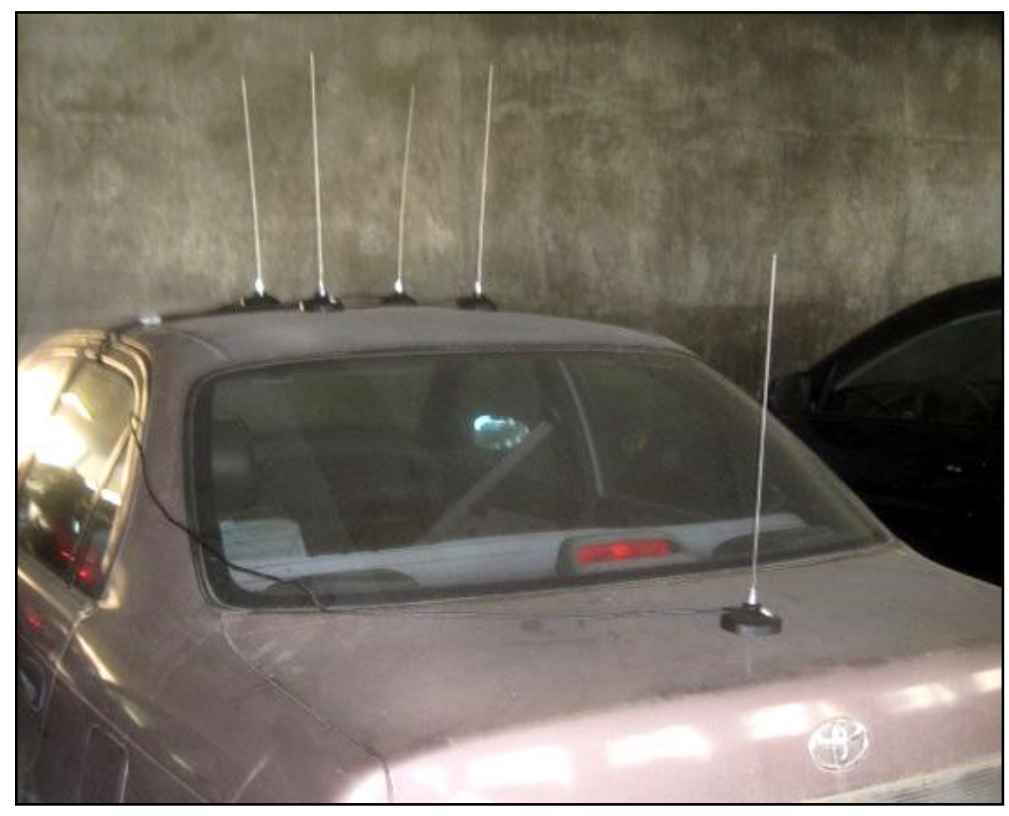

Figure 30: Pseudo-Doppler RDF test system vehicle installation 
Numerous test headings were measured from sources including the NOAA Weather Station on Cuesta Peak operating on 162.550 MHZ, a local handheld transmitter on $146.5 \mathrm{MHz}$, and several VHF amateur radio repeaters including 146.67 MHz (Tassajara Peak), $147.36 \mathrm{MHz}$ (Mt Lowe), and $146.700 \mathrm{MHz}$ (Arroyo Grande). Figure 31 shows a strong signal from the Mt Lowe repeater with a single, prominent zero-crossing on the right providing an accurate (but uncalibrated) heading indication.

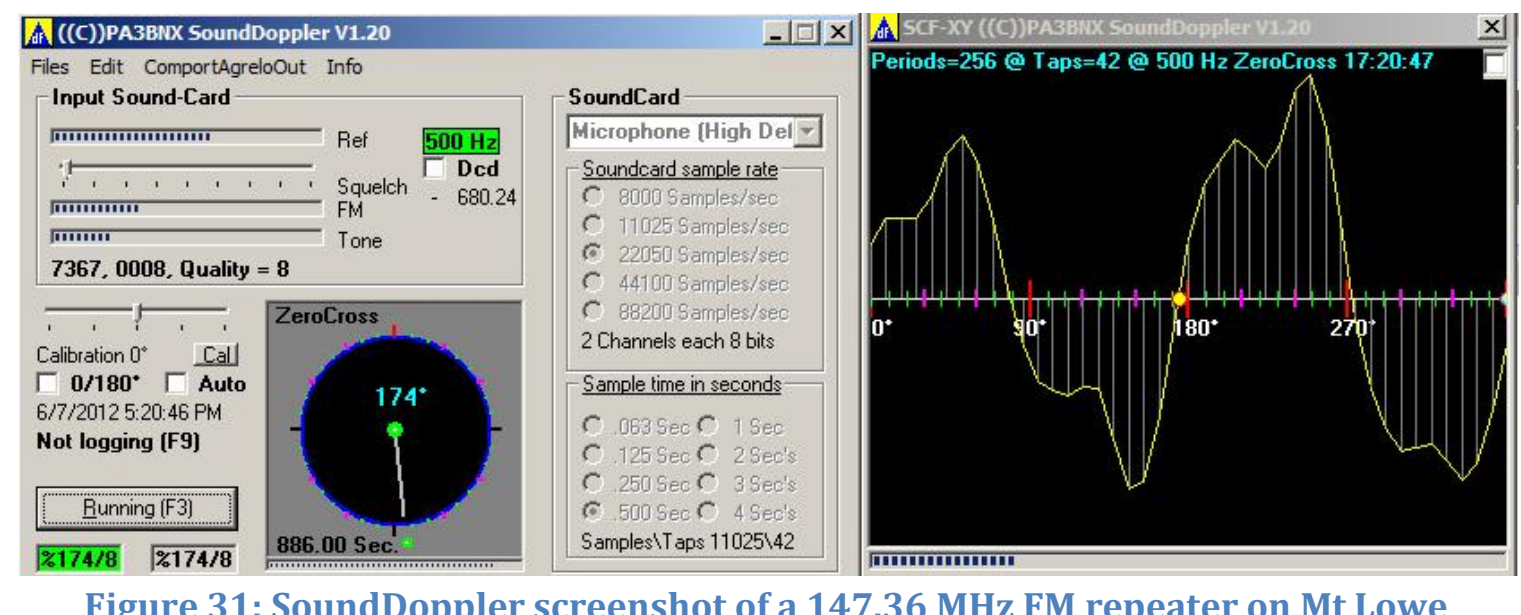

Tests were performed by driving around the greater San Luis Obispo area and observing the reported headings from the SoundDoppler interface. While the headings produced by the array were generally within $10^{\circ}$ of the actual signal direction, variations of up to $30^{\circ}$ were observed as the vehicle traveled through peaks and nulls caused by multipath. This was especially noticeable on straight roads (e.g Los Osos Valley Road) where the expected signal heading should not change by more than a few degrees but variations were visible. This is a known phenomena for mobile pseudo-Doppler arrays and typically amateur radio operators using this equipment become skilled at reading the output from the device and averaging the reported headings to provide a more accurate signal heading. At a fixed site, the multipath only changes when the transmitting station moves, so the variation will remain in the signal. 


\subsection{Pseudo-Doppler Results}

The pseudo-Doppler RDF system proved to be successfully developed into a microcontrollerdriven DSP proof-of-concept that is able to receive and calculate signal directions. One major problem with the prototype is that of multipath distortion that occurs when the source signal reaches the Doppler array time-delayed. This causes additional zero-crossings in the DSP stage and produces erroneous readings such as those in Figure 32, where the received signal is $>30^{\circ}$ off from the actual bearing. Further development of the system could include implementing the DSP software in the Arduino UNO, though a different microprocessor might be needed due to the limited processor power and storage on the ATMega328P.

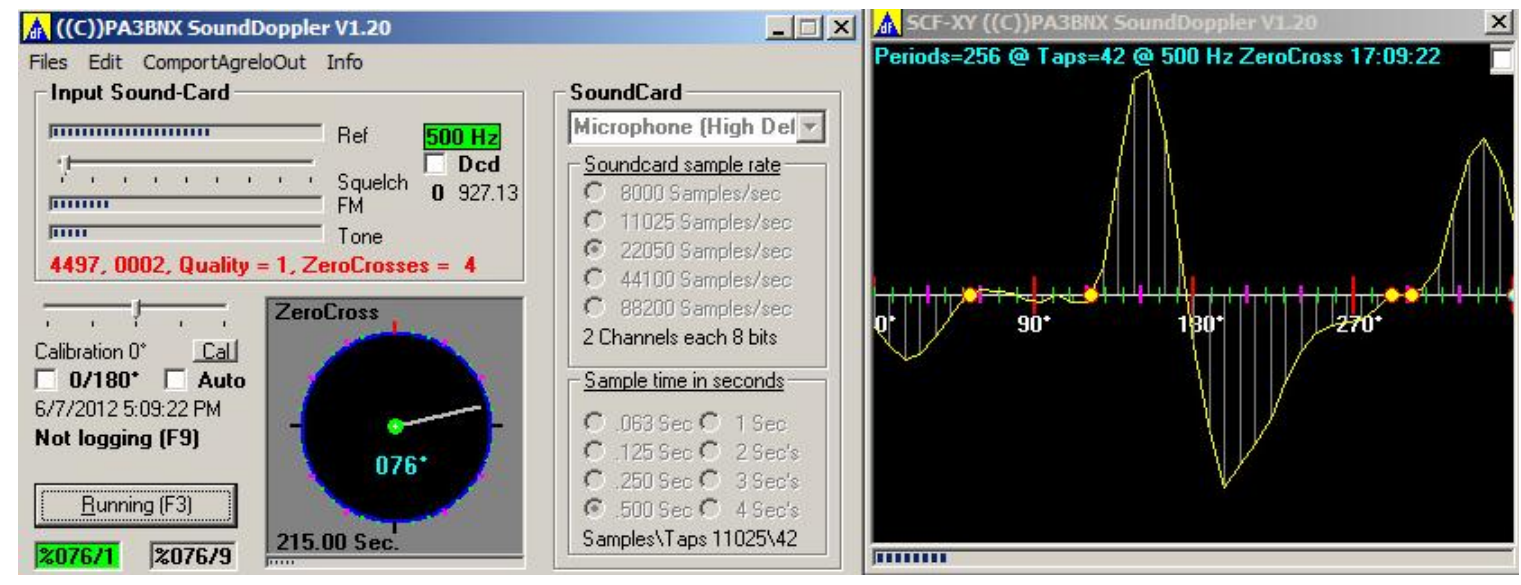

Figure 32: SoundDoppler screenshot of a $146.5 \mathrm{MHz}$ local transmitter with multipath distortion 


\subsection{Conclusion on the Pseudo-Doppler Receiver}

The unpredictable multi-path errors, large antenna array, and complicated system topology make the system unsuitable. The system error was observed up to $30^{\circ}$ from the desired bearing. The antenna array was approximately $20 " x 20 " x 20 "$ for the VHF system and requires a magnetic ground plane for proper operation. Even with a simplified antenna switcher and a modernized control circuit, the system is complicated and can quickly become expensive after final integration into a deployable package. The pseudo-Doppler RDF receiver is conceptually a very capable system, but in practice is quite complex to develop and implement. Given the design criteria of this project, the pseudo-Doppler system is quickly found to lose footing as a viable solution. For these reasons, the pseudo-Doppler system does not meet the primary problem specifications for low-cost and simplicity and is therefore no longer a viable solution. 


\section{Time Difference of Arrival Direction Finding Prototype}

Given the limitations and complexities of the pseudo-Doppler DF system, a 2nd prototype system was investigated for evaluation. A primary limitation of the pseudo-Doppler DF system for this project is the construction requirements that greatly complicate assembly and implementation. The next system was chosen primarily due to its low-dependence on any non-commercial component integration, though this comes with several constraints including limited accuracy and more complex data processing.

\subsection{TDOA Overview}

Time difference of arrival (TDOA) direction finding relies on the finite speed of electromagnetic waves in the atmosphere. Conceptually, if one can precisely determine when a signal was received at multiple time-coordinated receive sites, the location of a transmitter can be determined based on the time of flight (ToF) of that signal to each receiver. The accuracy of these measurements is primarily dependent on timing accuracy of these systems and on the level of precision that the signal's arrival can be determined [43]. Accurate timing in a deployable system is challenging given the distances between sites and the remote nature of the network. The most practical method for time-syncing the receivers is through use of GPS satellite timing. Note that the GPS receivers are only used on the receiver sites and not recommended for use on each asset to track as that would be prohibitively expensive and not meet the design criteria. 


\subsection{GPS Clock Accuracy and Issues}

This design used COTS GPS receivers for the system timing since they are easy to obtain and incorporate into the system. One of the primary issues with using COTS GPS receivers to provide the time-base for a TDOA is in their timing accuracy and precision. Typical consumer-level GPS receivers only have $1 \mu$ s timing accuracy [44] and often only report to 0.1 s or $1 \mu$ s precision per the NMEA 0183 standard for GGA fix data packets [45] (See also Appendix B: NMEA 0183 GGA Packet Format). Through the simple calculation shown below, these timing inaccuracies can produce up to 300m error from GPS timing accuracy alone (not including additional delays and errors from the rest of the receiver system).

$$
D_{\text {error }}=c \cdot t_{\text {error }}=\left(3 \times 10^{8} \frac{\mathrm{m}}{\mathrm{s}}\right)\left(1 \times 10^{-6} \mathrm{~s}\right)=300 \mathrm{~m} \text { error }
$$

In order to provide greater locational accuracy, the total system error needs to be lower than $1 \mu \mathrm{s}$. An appropriate target for this project is a $100 \mathrm{~m}$ error which would require $333 \mathrm{~ns}$ total timing accuracy as shown in the equation below.

$$
t_{\text {error }}=\frac{D_{\text {error }}}{c}=\frac{100 \mathrm{~m}}{3 \times 10^{8} \frac{\mathrm{m}}{\mathrm{s}}}=333 \text { ns error }
$$

Some commercial timing systems are available that use more sophisticated GPS receiver systems to produce timing accuracy up to $100 \mathrm{~ns}$ [46]. This would allow for a sub-50m system accuracy. The problem with these high-precision receivers is their high-cost $(\$ 2000+)$ and larger size, both of which are major limitations from the project specification. It is also important to recognize that receive site timing accuracy is only one part of the total system precision. Additional factors may cause errors in the location estimation including multipath, receiver delays, and receiver locations with respect to the target [43]. 


\subsection{TDOA System Configuration}

The COTS TDOA implementation is surprisingly simple in its hardware configuration. The entire system only requires a radio transceiver, a GPS module, and some form of microcontroller to interface between them. Since the proof-of-concept was to integrate with the amateur radio APRS network, finding components to build this prototype focused on the same companies that manufacture equipment for the amateur radio community. Argent Data Systems (ADS) is located in Santa Maria, CA and manufactures one of the world's most widely used APRS systems known as the Open Tracker series [47]. The Open Tracker is a device that interfaces a radio with the APRS network and controls everything from packets handling and audio levels to telemetry and GPS interfacing. ADS is focused on creating highquality, low-cost, and open-source hardware for the amateur radio community, but also supplies commercial fleet management companies that utilize Garmin's Fleet Management Interface to coordinate their vehicles on the road. Given these factors, the TDOA prototype was designed from ADS components in order to simplify the system. It should be noted that equivalent system components are available from other manufacturers (e.g. Byonics [48]) but with potentially reduced functionality. The Open Tracker series comes in a variety of form factors for different applications, but for this project the newer T3-301 was chosen because it contains both a Tracker3 module and a 5 watt VHF transceiver in one compact package. The simplicity of this form factor specifically targets one of the project's design criteria and the availability of all the components for the project from one manufacturer exemplifies this. 
The following list includes all of the components required for the TDOA system including the power source (battery) and GPS receiver.

\subsubsection{Equipment List}

- $\quad$ Argent Data Systems T3-301 Integrated VHF Data Radio (\$229)[49]

- Argent Data Systems Tracker 3 APRS Tracker

- Friendcom FC-301/D 5 watt VHF Data Transceiver

- Argent Data Systems ADS-GM1 SiRFstar III GPS Module (\$45)[50]

- Argent Data Systems T2-301 Serial + Power Cable (\$8)[51]

- VHF Whip Antenna (\$9)[38]

- 12V 35Ah Werker Deep Cycle AGM Battery (\$87)[39]

- Anderson Powerpole connectors and battery wire (\$10)

\section{Total system cost: $\$ 388$}

The complete system is shown below in Figure 33 and includes all the components needed for the prototype. The total TDOA system cost is only $\$ 388$ vs. $\$ 500$ for the pseudo-Doppler system, a $22.4 \%$ savings. It is important to notice the simplicity of this implementation when compared to alternate solutions, specifically the pseudo-Doppler system.

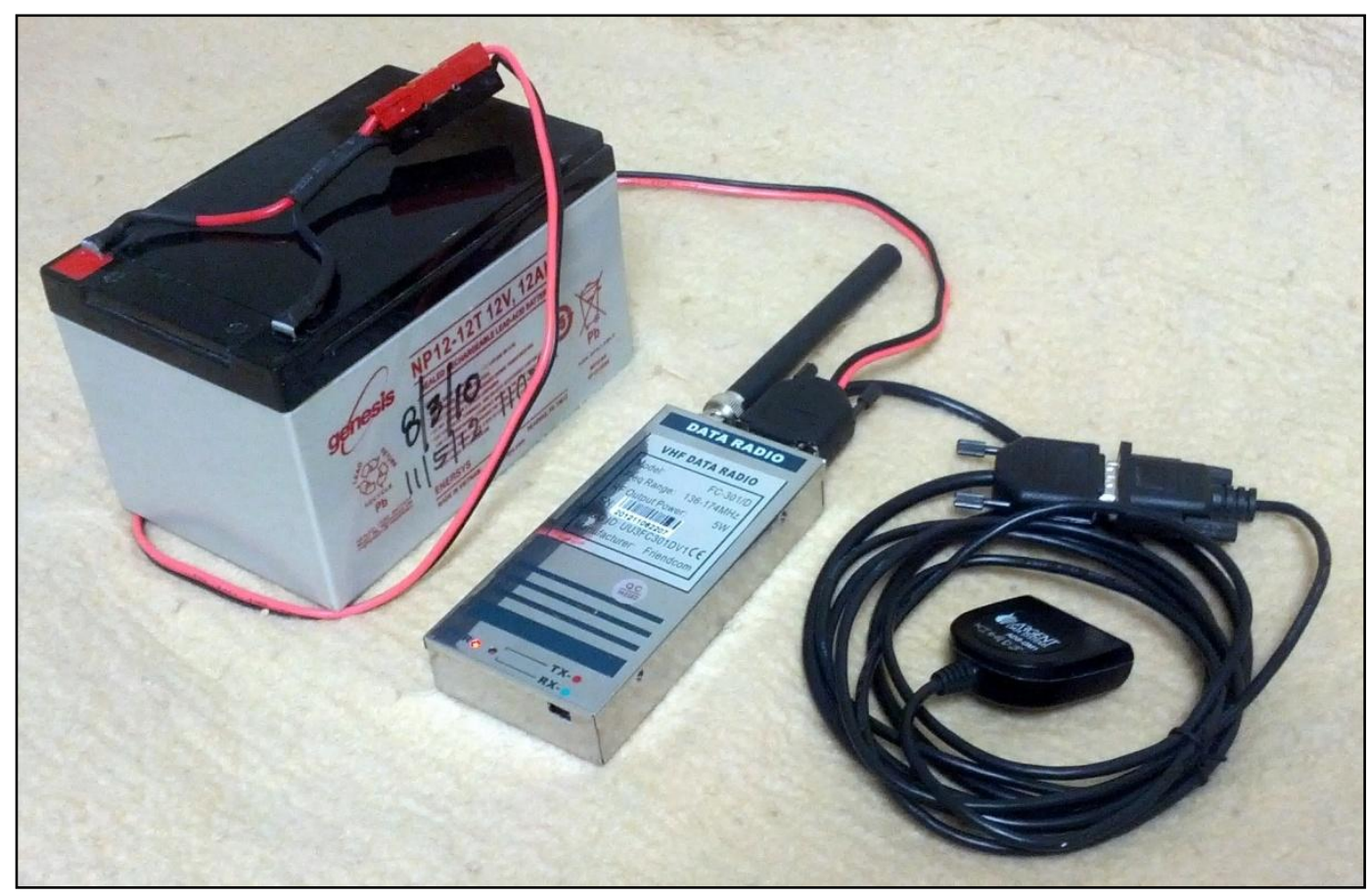

Figure 33: Complete COTS TDOA system with radio, battery, and GPS 


\subsubsection{TDOA System Block Diagram}

Figure 34 shows the TDOA system block diagram as built for this prototype. It is worth noting the simplicity of the design with very minimal component requirements. This is largely due to use of the T3-301 integrated Tracker3 and data radio module that reduces system complexity and cost by providing a combined solution.

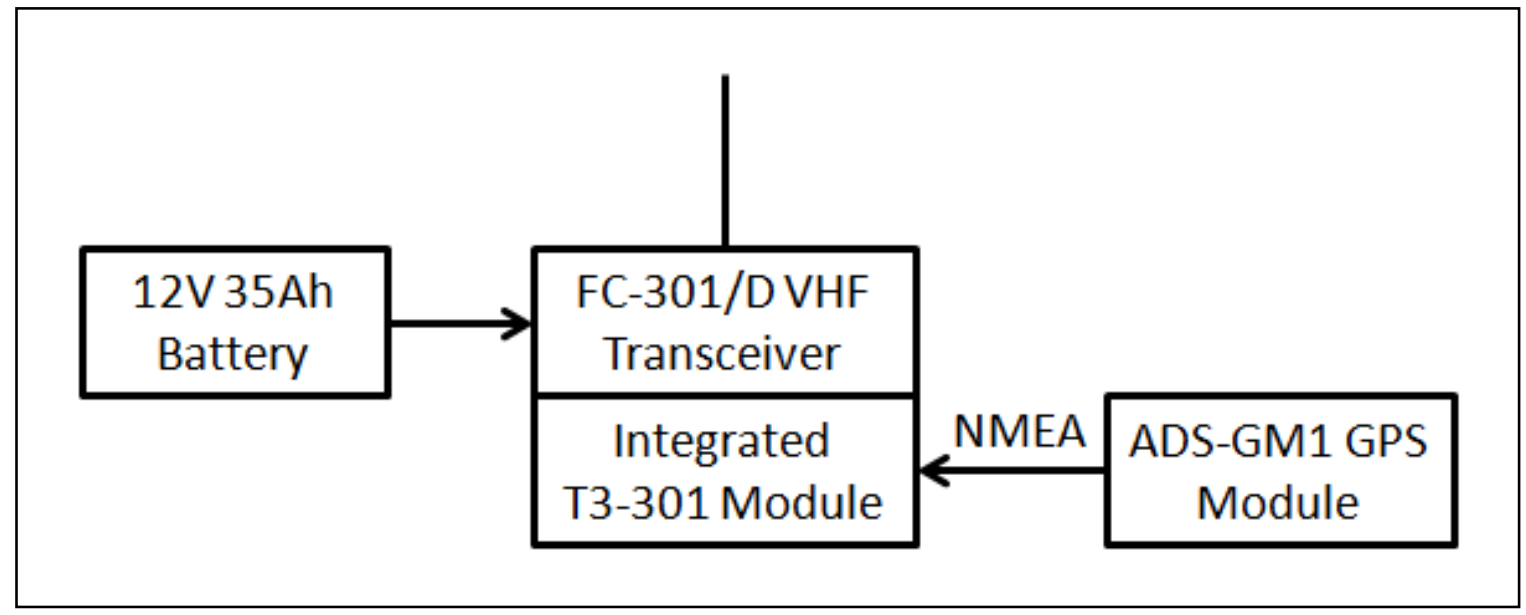

Figure 34: TDOA system block diagram 


\subsection{TDOA System Component Validation}

In order to analyze the contribution and durability of each component of the system, the subsections of the TDOA system are individually investigated.

\subsubsection{Power Analysis}

In order to meet the 1 week (168 hour) deployment requirement, each receiver unit needs sufficient battery power to continue operation. A power budget is analyzed below in Table 2 to estimate the total required system power capacity.

\section{Table 2: TDOA System Power Budget Analysis}

\begin{tabular}{|c|c|c|c|c|}
\hline Component & $\begin{array}{l}\text { Operating } \\
\text { Voltage }\end{array}$ & Measured Current & $\begin{array}{l}\text { Power } \\
\text { Use }\end{array}$ & $\begin{array}{l}\text { Total Power } \\
\text { (168 hours) }\end{array}$ \\
\hline $\begin{array}{l}\text { FC-301/D VHF Data } \\
\text { Transceiver with T3- } \\
301 \text { module }\end{array}$ & $12.5 \mathrm{~V}$ & $\begin{array}{l}\text { 115mA (Standby, 80\%) } \\
\text { 124mA (Receive, 10\%) } \\
0.71 \mathrm{~A} \text { (Transmit, 1W, } \\
10 \% \text { ) }\end{array}$ & $2.19 \mathrm{~W}$ & 367.9 Whrs \\
\hline ADS-GM1 GPS Receiver & $5 \mathrm{~V}$ & $32 \mathrm{~mA}$ & $0.16 \mathrm{~W}$ & 26.9 Whrs \\
\hline
\end{tabular}

A total system power usage of 394.8 Whrs is needed and corresponds to approximately 31.6 Ah for a $12.5 \mathrm{~V}$ nominal system. A 12 Ah lead-acid battery was chosen for the proof-ofconcept prototype due to its size and low-cost. The battery used for the project is shown in Figure 35. The 35Ah battery required would be approximately three times the size.

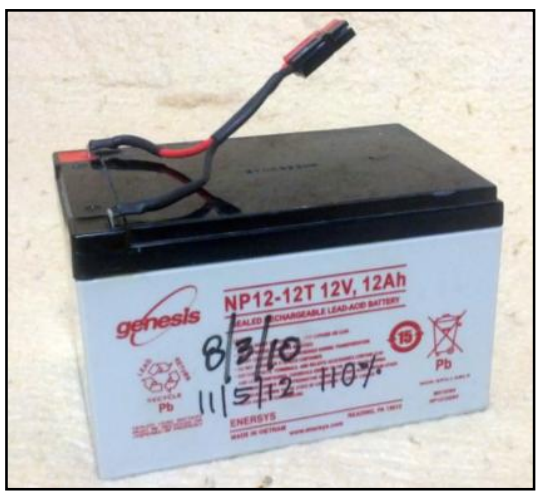

Figure 35: Genesis 12V 12Ah sealed lead-acid battery 


\subsubsection{Friendcom FC-301/D VHF Transmitter Characterization}

The data radio transceiver chosen for this project is the Friendcom FC-301/D 5 watt VHF module (Figure 36). This radio was chosen in part due to its FCC Part 90 certification that allows for operation on commercial frequencies [52]. This greatly improves the target audience for the project since low-cost amateur radio transceivers (Part 97 approved) are not rated for commercial use and therefore could only be used by licensed amateur radio operators and not, for example, by public service or search and rescue teams. Part 97 approved radios typically have lower performance specifications that are compromised to meet a low price point. While an amateur radio transceiver can easily be purchased for less than $\$ 100$ (vs. $\$ 149$ for the bare FC-301/D module), the estimated 50-75\% increase in cost for this commercial-rated and data-specific radio module was determined valuable for this project since it broadens the potential target audience.

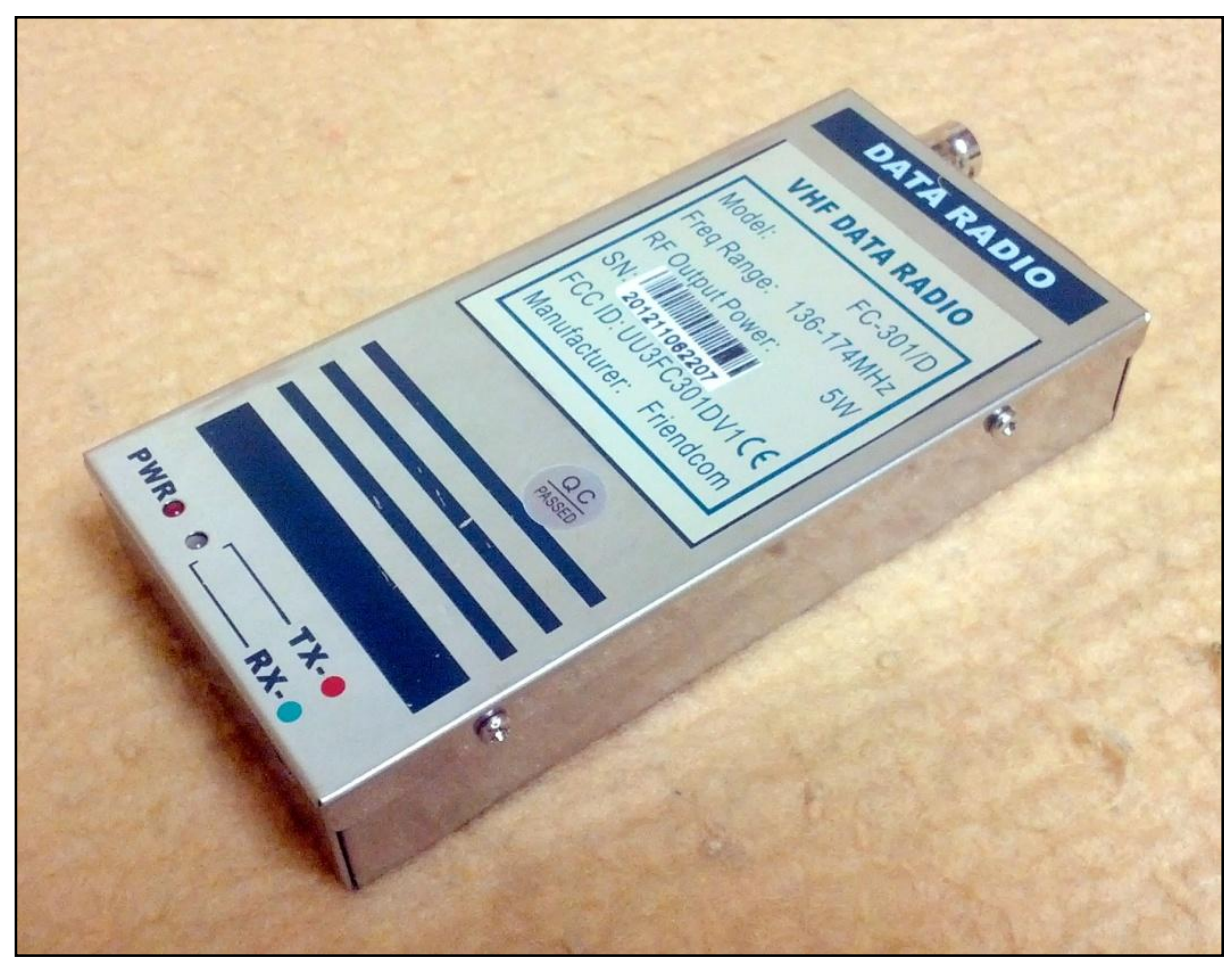

Figure 36: Friendcom FC-301/D VHF data radio 


\subsubsection{Verification of Radio Specifications}

The FC-301/D data radio was characterized against the noted specifications to verify correct operation and compliance for commercial operation per FCC Part 90 specifications. Table 3 shows the specified characteristics of the transceiver as stated in the radio service manual [53] and then the corresponding measured value. All measurements were performed on an Agilent CXA N9000A Spectrum Analyzer and a Motorola R2200 Communications Service Monitor. The T3-301 was set to transmit alternating $1200 \mathrm{~Hz}$ and $2200 \mathrm{~Hz}$ tones for the transmission tests as are used for APRS packets [54].

Table 3: FC-301/D Data Radio Characterization

\begin{tabular}{|c|c|c|c|}
\hline Category & Metric & Specified Value[53] & Measured Value \\
\hline \multirow{3}{*}{$\begin{array}{l}\text { Power Usage } \\
\text { (at } 12.5 \mathrm{~V} \text { ) }\end{array}$} & Standby (muted) & $<40 \mathrm{~mA}$ & $\begin{array}{c}120 \mathrm{~mA} \text { (includes T3- } \\
301 \text { current) }\end{array}$ \\
\hline & Transmit 5 Watts & $<1.5 \mathrm{~A}$ & $1.16 \mathrm{~A}$ \\
\hline & Transmit 1 Watt & $<0.8 \mathrm{~A}$ & $0.71 \mathrm{~A}$ \\
\hline \multirow{4}{*}{$\begin{array}{c}\text { Transmitter } \\
\text { Characteristics } \\
\text { (at } 144.99 \mathrm{MHz} \text { ) }\end{array}$} & $\begin{array}{l}\text { Frequency Error } \\
\text { (@144.990MHz) }\end{array}$ & $\begin{array}{l} \pm 2.5 \mathrm{ppm} \\
( \pm 362 \mathrm{~Hz})\end{array}$ & $\begin{array}{c}+0.3-0.8 \mathrm{ppm} \\
(+50-120 \mathrm{~Hz})\end{array}$ \\
\hline & Frequency Deviation & $< \pm 5.0 \mathrm{kHz}$ & $\begin{array}{c} \pm 3.2 \mathrm{kHz} \\
\text { (programmable) }\end{array}$ \\
\hline & $\begin{array}{l}\text { Adjacent Channel } \\
\text { Power }\end{array}$ & $\begin{array}{l}<-70 \mathrm{dBc} \text { nominal } \\
\text { (25kHz spacing) }\end{array}$ & $-70.9 \mathrm{dBc}$ (average) \\
\hline & $\begin{array}{l}\text { 2nd Harmonic } \\
\text { Spurious Level }\end{array}$ & (Not given) & $\begin{array}{c}-69.9 \mathrm{dBc} \\
\text { (at } 289.98 \mathrm{MHz}) \\
\end{array}$ \\
\hline \multirow{3}{*}{$\begin{array}{c}\text { Receiver } \\
\text { Characteristics } \\
\text { (at } 144.99 \mathrm{MHz} \text { ) }\end{array}$} & $\begin{array}{c}\text { RX Sensitivity } \\
(12 \mathrm{~dB} \text { SINAD*) }\end{array}$ & $<-118 \mathrm{dBm}$ & $-122 \mathrm{dBm}$ \\
\hline & $\begin{array}{l}\text { Squelch Opening } \\
\text { Sensitivity }\end{array}$ & $-118 \mathrm{dBm}$ & $-122 \mathrm{dBm}$ \\
\hline & $\begin{array}{c}\text { Squelch Closing } \\
\text { Sensitivity }\end{array}$ & $-121 \mathrm{dBm}$ & $-123 \mathrm{dBm}$ \\
\hline \multirow{3}{*}{$\begin{array}{l}\text { Power Output } \\
\text { (at } 12.5 \mathrm{~V} \text { ) }\end{array}$} & Low Power & $1 \mathrm{Watt}$ & 1.44 Watts \\
\hline & Medium Power & 3 Watts & 3.38 Watts \\
\hline & High Power & 5 Watts & 5.21 Watts \\
\hline
\end{tabular}

*SINAD - SIgnal to Noise And Distortion ratio 


\subsubsection{Spectral Verification}

The output spectrum of the FC-301/D transceiver was measured at $144.990 \mathrm{MHz}$ and 1 watt RF power on the Agilent CXA N9000A Spectrum Analyzer and is shown in Figure 37. This was done to verify the spectral content of the data radio at the transmission frequency. It is important to note that the T3-301 is sending a 1200baud AFSK audio signal (alternating 1200 and $2200 \mathrm{~Hz}$ [55]) to the FM radio module so the actual spectrum shown is roughly a two-tone FM signal with audio harmonics visible on the signal skirts.

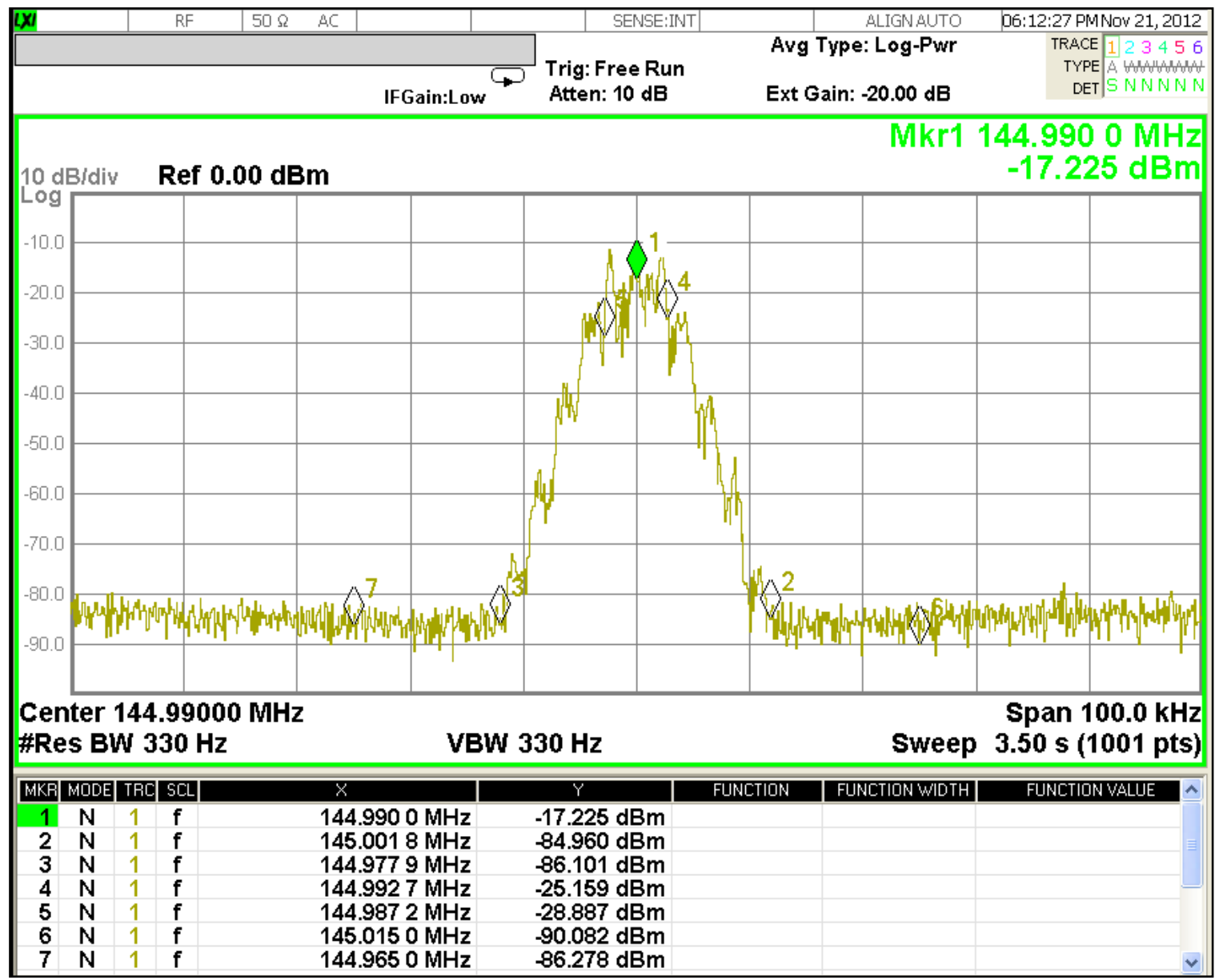

Figure 37: FC-301/D transceiver output spectrum at 144.990MHz (1 watt) 


\subsection{T3-301 APRS Module Configuration}

The brains of the TDOA are in the T3-301 module shown in Figure 38. This device is based on a 32-bit Coldfire MCF51JM128 processor and features built in telemetry and digipeater functionality [6]. It was designed by Scott Miller N1VG from Argent Data Systems. The processor includes 2 serial ports for direct interfacing with a GPS, weather station, or a computer. The T3-301 was designed to function on the APRS network and therefore includes all packet handling, location reporting, and telemetry features in the standard firmware. It also includes a basic scripting language that can be used to implement additional functionalities [56].

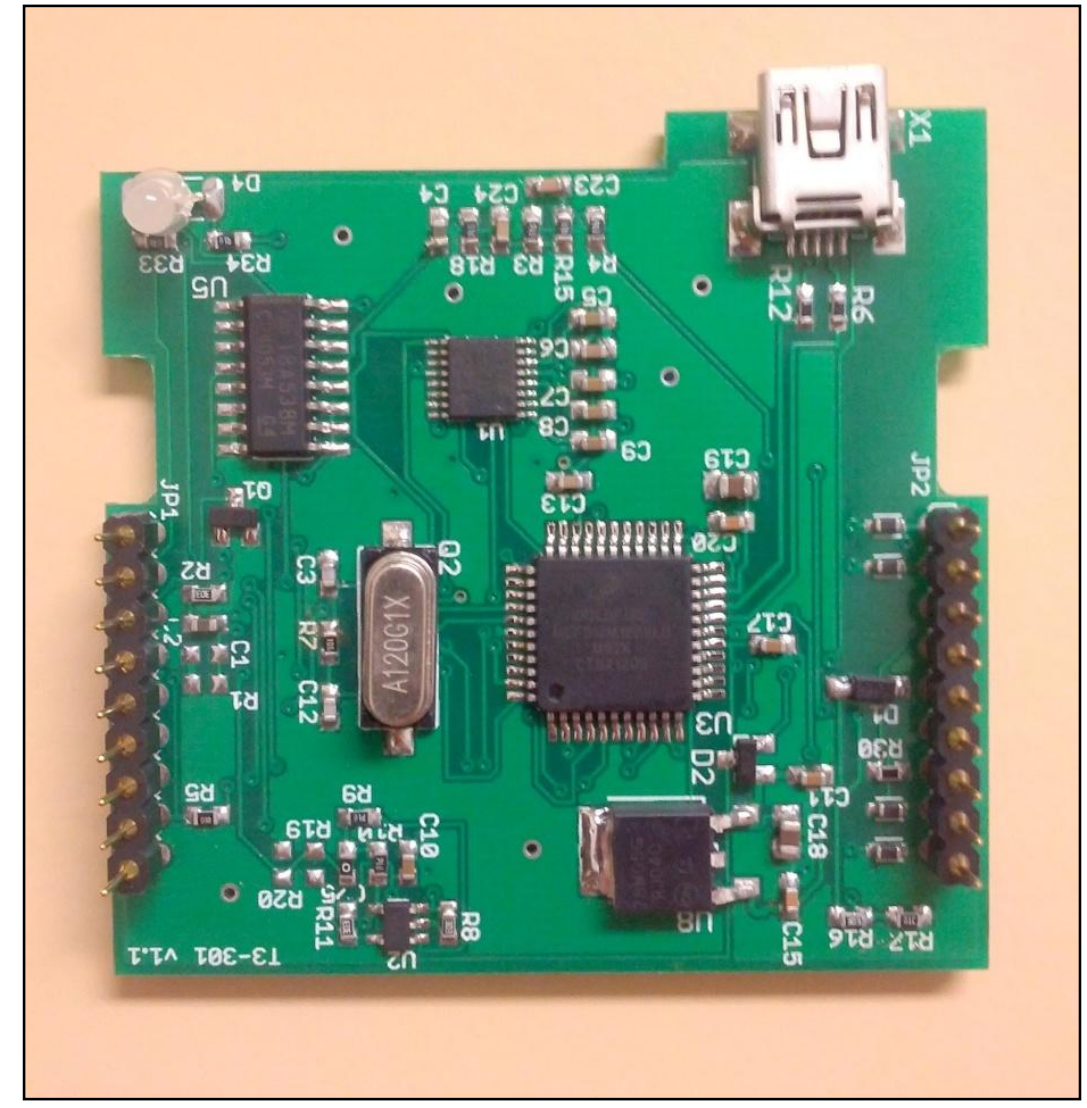

Figure 38: Argent Data Systems T3-301 Tracker Module v1.1 


\subsubsection{T3-301 Configuration Software}

The entire Open Tracker series of devices from Argent Data Systems uses a configuration utility that runs on Windows called OTWINCFG. This program is a user friendly configuration software that allows the user to adjust all parameters of the device including reporting telemetry, transmit audio levels, digipeating, packet routing, and device interfaces. The screenshot in Figure 39 shows the settings used for this test device. The notable settings are the built-in "Reporting Options" on the right side.

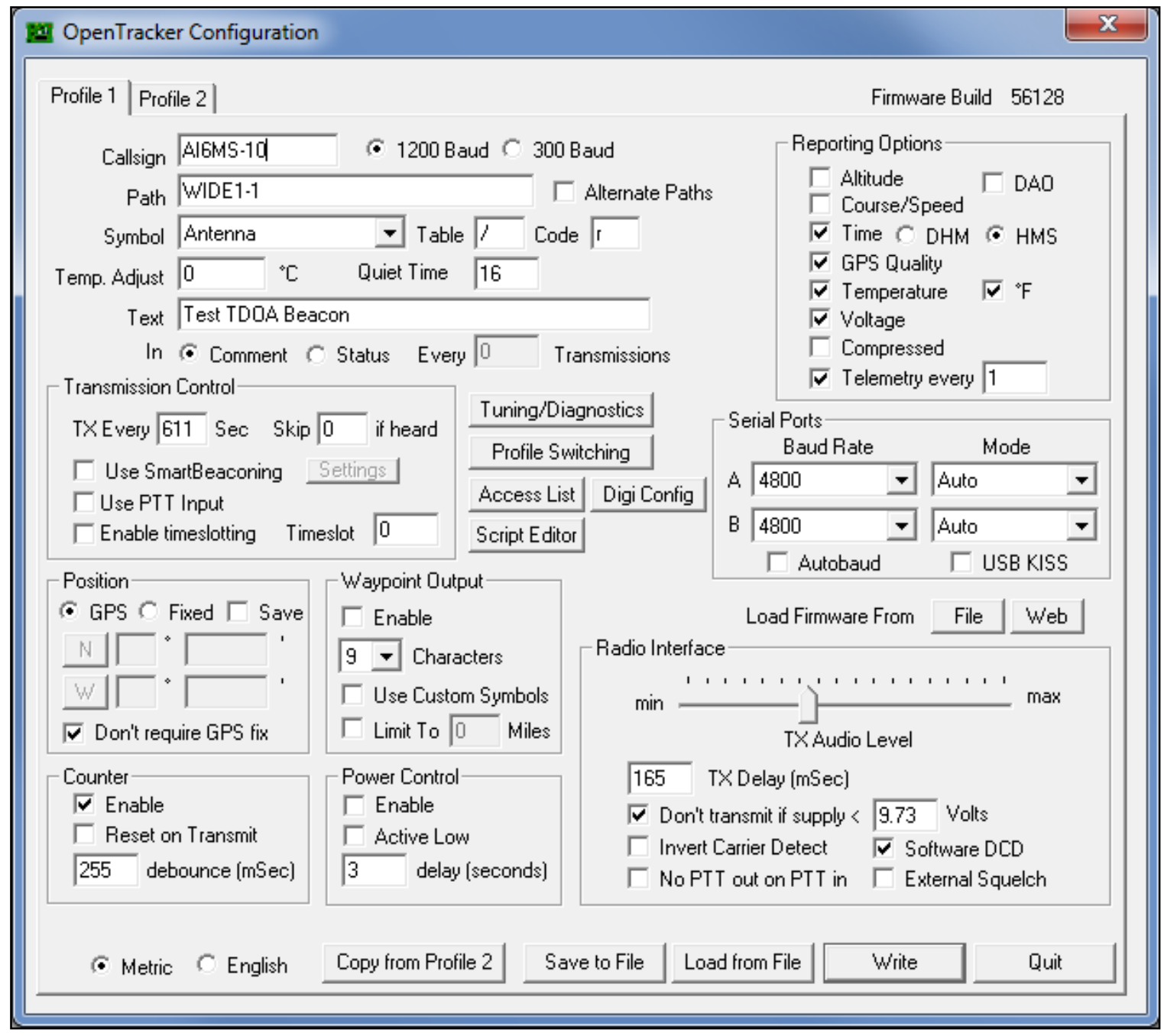

Figure 39: OTWINCFG T3-301 Configuration Utility 


\subsubsection{T3-301 Device Interfacing}

The TDOA receiver needs to activate anytime it hears a signal on the RX frequency and then transmit the signal metrics of time received and RSSI to the TX frequency. While the T3-301 has the capability from a hardware perspective to do this, the current firmware does not allow direct access to the RSSI and Squelch (SQL) data lines on the radio module. Since writing a custom firmware for the processor was out of the scope of this project, a simple hardware workaround was developed to send the SQL data to interrupt pin and allow use of the scripting engine for the reporting. This involved a simple jumper wire on the back of the T3-301 from pin 6 of connector JP1 to pin 6 of connector JP2 as shown in Figure 40 (The schematic diagram for the T3-301 is shown in Appendix C: Argent Data Systems T3-301 Schematic ). This jumper wire simply connects the squelch active-low signal to the IRQ interrupt pin on the T3-301. The result is that anytime the tracker receives a signal that breaks squelch, the interrupt routine can be invoked by the scripting engine.

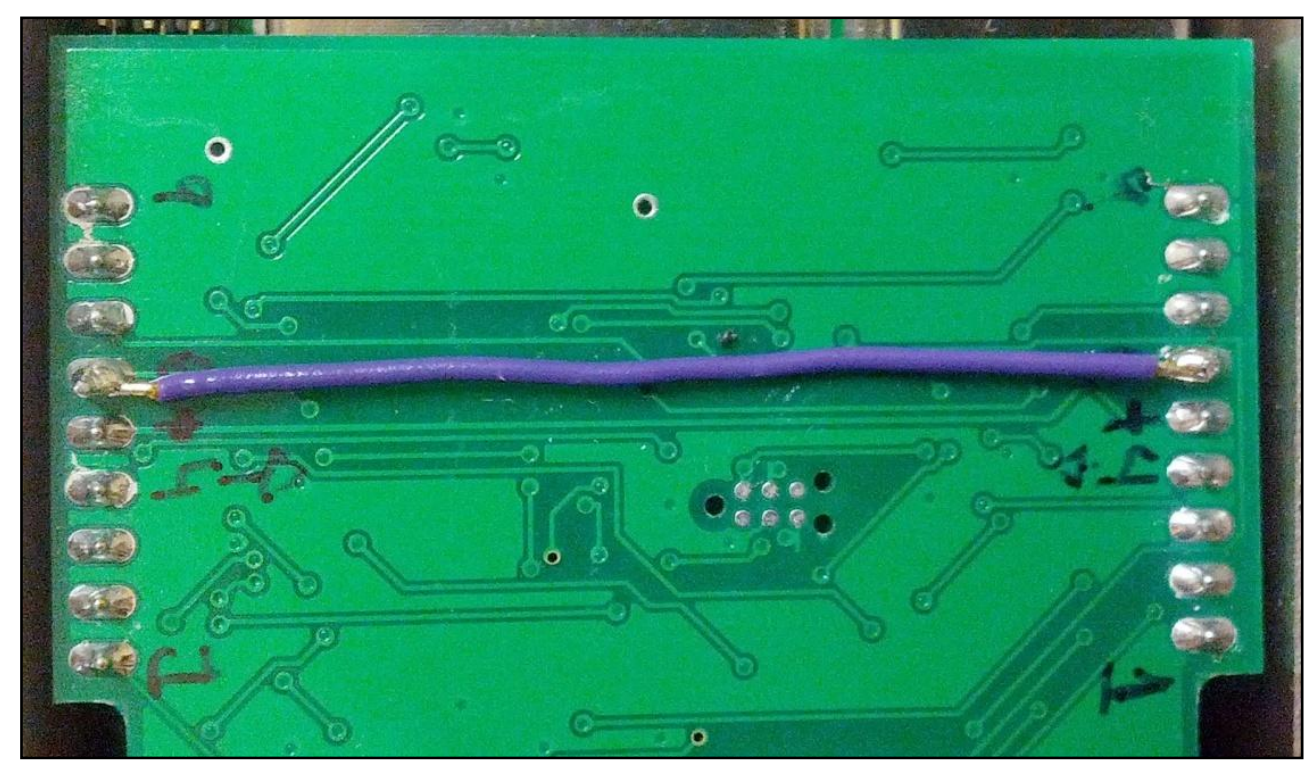

Figure 40: Squelch to IRQ jumper wire on the back of the T3-301 
The rest of the implementation is surprisingly simple, only 3 lines of scripting code are required to have the device send out a time-stamped beacon when the interrupt pin is triggered. The scripting editor and lines of test code are shown in Figure 41. The only other important configuration is shown in Figure 39 to enable the counter and set the debounce to the maximum $255 \mathrm{~ms}$. This prevents the unit from self-triggering the interrupt when it transmits the data packet since the SQL line triggers when the device transmits as well.

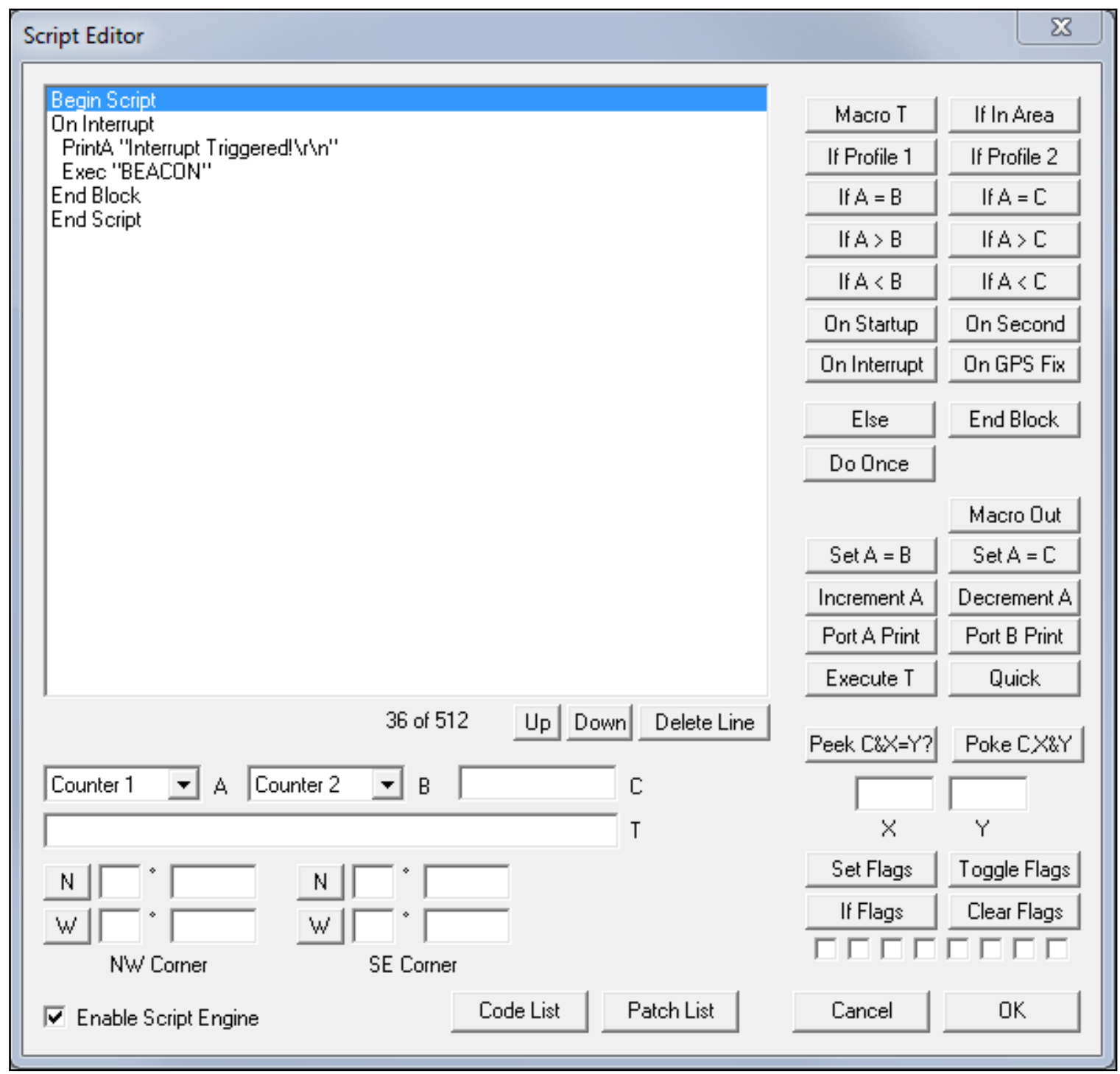

Figure 41: 0TWINCFG Script Editor 


\subsection{TDOA System Results}

Basic functionality of the TDOA system was proven in the following test. The current implementation is complete from a hardware perspective but requires additional software and firmware development to have full system-level functionality.

\subsubsection{APRS Packet Results}

The TDOA system responds immediately to any on-frequency traffic as designed and transmits the following packet information each time:

- AI6MS-10>APOT30,WIDE1-1:>Test TDOA Beacon

- AI6MS-10>APOT30,WIDE1-1:/231915h3518.51N/12039.56Wr $12.6 \mathrm{~V}$ 72F CNT00003 HDOP02.2 SATS06

- AI6MS-10>APOT30,WIDE1-1:T\#000,126,057,062,052,048,00000010

The first packet shows a simple status packet from the system to properly identify the station and stay in compliance with FCC Part 97 rules during testing. The second packet is the standard BEACON packet from the T3-301 and contains time, GPS latitude and longitude, system voltage, temperature, packet count, HDOP, and the number of satellites used. The primary issue with this packet is the imprecision of the reported time (further discussed in section 7.6.3 COTS Timing Challenges). The third packet shows a standard telemetry packet as reported by the T3-301. The basic information presented is the packet ID number, system temperature, voltage, ADC value, IRQ counter, and HDOP value. These are presented on a linear scale from 0 to 255. Further documentation on this format is found in the Argent Data Systems website and is the format in which RSSI could easily be reported via software changes to the T3-301 [57]. 


\subsubsection{COTS Hardware Implementation}

The primary challenge in creating a low-cost TDOA system for this project is in maintaining the COTS implementation requirement for simple construction and deployment capabilities. In order for the RDFN to successfully meet the project requirements, it needs to be easily assembled from commercially available components. One of the drawbacks of the current implementation is the necessity for a hardware jumper wire to activate the signal triggering. The hardware jumper also means that the device cannot be easily reconfigured for alternate operation without physically removing this wire. Fortunately, the device is capable of implementing the jumper in software and a request has been made to Argent Data Systems to develop this code for open source implementation in the future.

\subsubsection{COTS Timing Challenges}

The second issue with the current implementation is in obtaining the necessary timing accuracy for precise locating capabilities. As the prototype stands now, the reported time is only reported to 1 second precision as is default in the code. This does not provide enough accuracy for TDOA calculations unless the time of arrival can be calculated with respect to the second using a counter or something similar. Again with some small changes to the firmware, at least $1 \mathrm{~ms}$ precision with $1 \mu$ s accuracy can be obtained from the GPS unit and reported along with rest of the signal metrics. Another useful piece of data that is currently transmitted is the horizontal dilution of precision (HDOP). HDOP is a measure of the GPS location accuracy and can be used in calculating the probable transmitter location [58]. Depending on the exact GPS module used, there is potential for additional GPS signal reporting of time and vertical dilution of precision (TDOP and VDOP), clock drift, and clock frequency, all of which could be used by the central receive station to achieve even greater

signal timing accuracy [59]. These changes are all software based implementations that are further addressed in Section 8.2 Future Project Developments. 


\subsubsection{TDOA Network Implementation}

A complete TDOA network can be built for under $\$ 2000$ with as little as three TDOA receiver sites and one base processing station as shown in Figure 42. The base processing station is simply an FM receive radio connected to a computer (e.g. through the sound card) that is running an APRS program such as AGWTracker [60] or APRSISCE/32 [61]. Both of these programs support APRS Direction Finding formatted packets [54] and can then plot locations based on this information. For the TDOA system, support for a time of arrival packet format would need to be added to these programs to enable automatic locating without any enduser requirements. The ideal implementation would simply plot known locations of various transmitters with confidence intervals and make the entire TDOA calculation transparent to the end user. The $1 \mu$ s timing accuracy of the GPS used in this project, signal locations could be determined to $333 \mathrm{~m}$ accuracy.

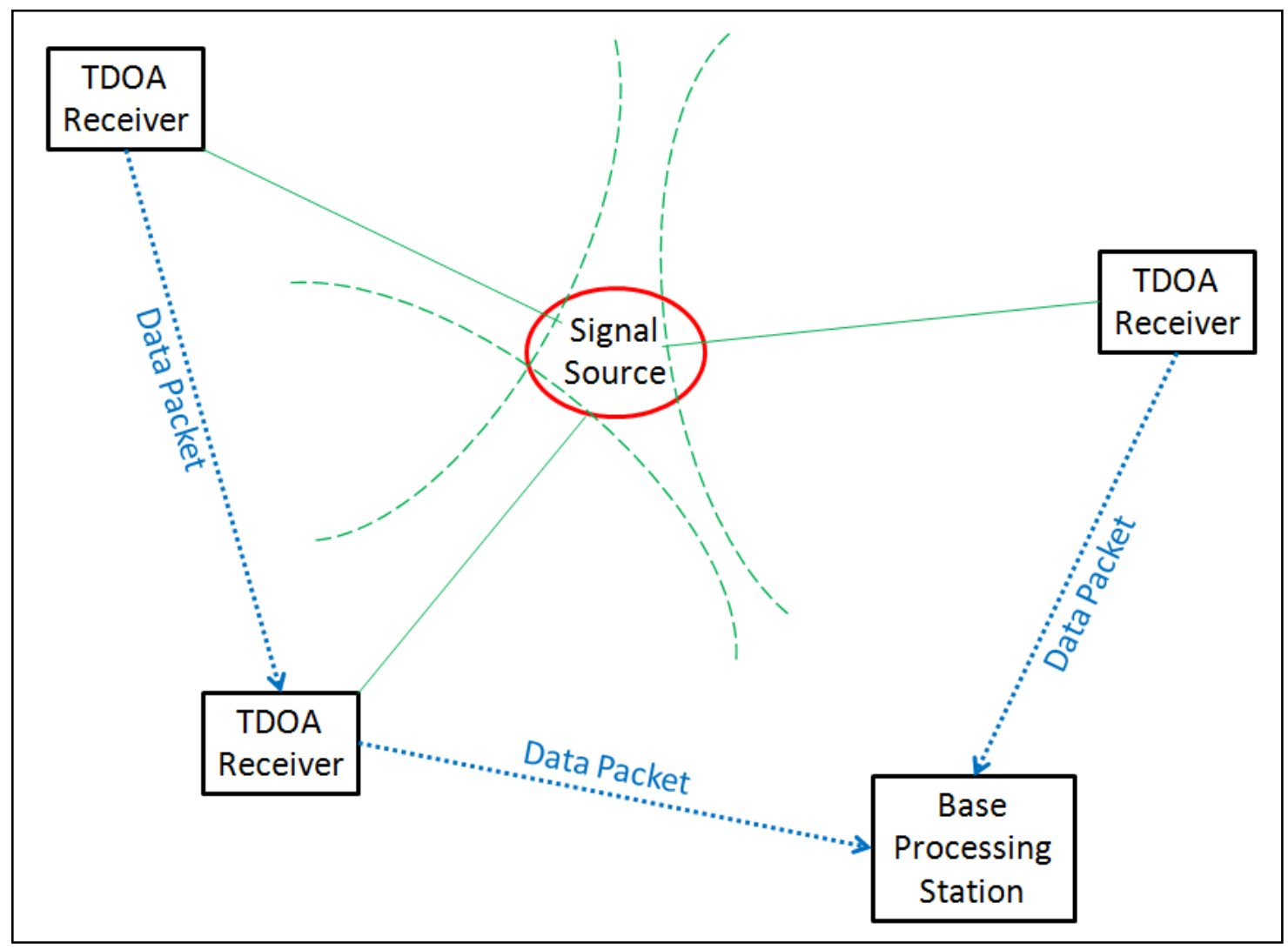

Figure 42: TD0A Network Configuration 


\section{Conclusion and Future Work}

This project is set out to provide the basic system-level testing and analysis to prove the feasibility of a low-cost, deployable, VHF radio direction finding network. In order to maintain the primary project goal of commercial off-the-shelf (COTS) construction, several design compromises were necessary to maintain cost and complexity requirements. A moreprecise (and thus higher-cost) system can easily be developed using more complex systems and custom hardware, but would ultimately fall outside the scope of this project. The pseudo-Doppler RDF receiver proved to require far more complex construction than was desired for this project and also proved to have directional accuracy errors of $30^{\circ}$ in certain situations. The TDOA RDF receiver proved to be exceptionally simple to assemble and was functional at a very basic level with minimal code requirement for the receiver unit. Additional software developments are necessary to make the TDOA receiver a practical solution for development into an RDFN. Additionally, the TDOA system uses a consumerlevel GPS that has clock accuracy resulting in greater than 300m locational error.

The feasibility of a low-cost, deployable RDF receiver was proven with the TDOA design which is implemented entirely from COTS components and has a total system cost of $\$ 388$. This proof-of-concept will likely see further development from future project work and find its way into regular use in public service and disaster response organizations worldwide. 


\subsection{Contributions}

This thesis project has made numerous contributions to the development of a low-cost COTS RDF receiver.

- Proven feasibility of a RDFN that is backwards compatible with existing handheld and mobile VHF radios currently used by public service groups with no other major infrastructure required to provide locating services.

- A low-cost system developed entirely from commercially available components is now possible at less than $\$ 500$ per receive site. This results in a minimum total system cost of under $\$ 1500$ with unlimited expansion capabilities and complete fault tolerance through redundant site usage.

- The RDFN is simple to implement and is based on an existing support network of amateur radio operators who are intimately familiar with the APRS network and already closely involved with public service through ARES and RACES.

- The author has worked closely with both Argent Data Systems to provide improvements and technical documentation support for the Open Tracker products.

- This thesis topic was presented by the author at the National Convention for Amateur Radio held at the Pacificon Convention in Santa Clara, CA on October 12-14, 2012 [62]. This presentation brought much feedback and positive response from the amateur radio community. Several amateur radio operators have expressed interest in the final product and are in communication with the author for future developments. Given the vastly positive response to this project, the author plans to publish his work in QST, the national magazine for amateur radio and also to work closely with several students who have already expressed interest at continuing the project research. 


\subsection{Future Project Developments}

Throughout the development of this project, numerous improvements and additional research areas were identified. These maybe of interest to future senior project or master's students and other individuals interested in contributing to the work. The following list briefly summarizes some of these areas for future project development:

- Improved Receiver Clock Accuracy and Precision

- Additional research into improving the receiver time synchronization would greatly improve the TDOA system viability. D-GPS could also be used to improve the timing accuracy. A detailed look at the system's clock distribution could find additional areas for improvement [43]

- $\quad$ Radio Transmitter Fingerprinting (via store-and-forward)

- A very promising area of study is in transmitter fingerprinting which allows for individual transmitters to be identified through their initial VCO locking characteristics as they are presented in the FM signal. This could allow for uniquely identifiable locating capabilities without the need for any additional equipment or infrastructure.

- Baseband RF Phase or Audio TDOA

- Instead of using the squelch of the transceiver (which has timing inaccuracies) to detect an incoming signal, one could measure the precise RF baseband signal or specific audio crossing point to improve accuracy.

- Environmental Fingerprinting (location calibration)

- This subject involves periodic system calibration using a series of known transmitter locations to characterize the coverage area and improve locating capabilities of the system. 
- Active user calibration (real-time calibration using known sources)

- As an extension from Environmental Fingerprinting, certain transmitters or fixed beacons could remain in the coverage area to actively recalibrate the system to maintain system accuracy over time and changing conditions.

- Frequency agile operation, remote reprogramming, start-up, and shutdown capable

- Technically this is already possible with the existing system by using the remote access feature of the T3-301, but further research can be done to take advantage of these features to provide active monitoring on multiple frequencies and systems.

- Indefinite stand-alone remote operation with solar power system

- A relatively simple improvement to this system is to add a photovoltaic panel to the system to improve the power system and allow indefinite operation.

- Networked weather reporting sites

- Another simple improvement to the RDFN is the addition of weather stations to provide real-time telemetry to the user. This is especially important for wildland firefighters who rely heavily on wind and temperature forecasts to allocate resources. The T3-301 has built in weather station capabilities so this improvement is quite trivial and would simply require added cost and receiver size to include the sensors.

- Commercialization

○ The final step for this project would be to bring it the product to market so that public service groups can easily take advantage of the system. Once the actual receiver accuracy is improved, the simple addition of a rugged enclosure would provide a promising product to use for an RDFN. 


\section{Bibliography}

[1] S. E. Lipsky, Mircowave Passive Direction Finding, New York: John Wiley \& Sons, Inc., 1987.

[2] J. F. Bull, "Wireless Geolocation," IEEE Vehiclular Technology Magazine, pp. 45-53, 2009.

[3] LoJack Corporation, "LoJack System for Cars, Trucks and Classics," 2012. [Online]. Available: $\quad$ http://lojack.com/Cars-Trucks-And-Classics/How-LoJack-Works. [Accessed November 2012].

[4] National Conference of Volunteer Examiner Coordinators, "Element 2 Technician Class Question Pool," 1 July 2010. [Online]. Available: http://www.ncvec.org/downloads/Revised\%20Element\%202.Pdf. [Accessed November 2012].

[5] R. Parry, "Position Reporting with APRS," [Online]. Available: http://www.arrl.org/position-reporting-with-aprs. [Accessed November 2012].

[6] Argent Data Systems, "Tracker3 Series APRS Tracker," 2012. [Online]. Available: https://www.argentdata.com/products/tracker3.html. [Accessed November 2012].

[7] National Oceanic and Atmospheric Administration, "Emergency Beacons," [Online]. Available: http://www.sarsat.noaa.gov/emerbcns.html. [Accessed November 2012].

[8] California Department of Forestry and Fire Protection, "Overview of the Accident," 11 May $2005 . \quad$ [Online]. Available: http://www.fire.ca.gov/fire_protection/downloads/404_investigation_3of5_mainpart2of2.pdf. [Accessed November 2012].

[9] P. Gething, Radio direction-finding and the resolution of multicomponent wavefields, London: Peter Peregrinus Ltd., 1978.

[10] K. B. Williams, Secret Weapon - U.S. High-Frequency Direction Finding in the Battle of the Atlantic, Annapolis: Naval Institue Press, 1996.

[11] RDF Products, "Prdocut Data Sheet Model DFA-1248B1," 2007. [Online]. Available: http://www.rdfproducts.com/dfa1248b1_pds_01.pdf. [Accessed November 2012].

[12] K. Halliwell, "NSGA Homestead, Florida," December 1970. [Online]. Available: http://www.navycthistory.com/homestead_KJH_1.html. [Accessed November 2012].

[13] United States Coast Guard, "Planning Process for the National Distress and Response System Modernization Project," 4 February 2002. [Online]. Available: 
http://www.oig.dot.gov/sites/dot/files/pdfdocs/mh2002076.pdf.

[Accessed November 2012].

[14] United States Coast Guard, "Acquisition Directorate Rescue 21," July 2012. [Online]. Available: http://www.uscg.mil/acquisition/rescue21/pdf/rescue21.pdf. [Accessed November 2012].

[15] United States Coast Guard, "Rescue 21: Image Gallery," 10 February 2004. [Online]. Available: http://www.flickr.com/photos/coast_guard/7204749914/in/set72157629497106120/. [Accessed November 2012].

[16] Civil Air Patrol National Headquarters, "Emergency Services," 2102. [Online]. Available: http://www.gocivilairpatrol.com/about/civil_air_patrols_three_primary_missions/e mergency-services/. [Accessed November 2012].

[17] L-Tronics Company, "LL Series Little L-Per Portable Direction Finder," January 2011. [Online]. Available: http://www.ltronics.com/ll-16df.htm. [Accessed November 2012].

[18] Spot LLC., "SPOT Satellite GPS Messenger," 2012. [Online]. Available: http://www.findmespot.com/downloads/SPOT2-SellSheet.pdf. [Accessed November 2012].

[19] Backcountry Access, "Tracker DTS," 2012. [Online]. Available: http://www.backcountryaccess.com/product/tracker-dts/. [Accessed November 2012].

[20] U.S. Geological Survey, "A Critique of Wildlife Radio-tracking and its Use in National Parks," Northern Prairie Wildlife Research Center, 3 August 2006. [Online]. Available: http://www.npwrc.usgs.gov/resource/wildlife/radiotrk/convent.htm. [Accessed November 2012].

[21] C. Ziegler, "Tiny Radio Tags Offer Rare Glimpse into Bees' Universe," National Geographic News, 14 November 2008. [Online]. Available: http://news.nationalgeographic.com/news/bigphotos/21191490.html. [Accessed November 2012].

[22] Conservacion Patagonica, "Huemul Deer Recovery," [Online]. Available: http://www.patagonialandtrust.org/buildingthepark_biodiversity_huemul.htm. [Accessed November 2012].

Motorcycle.com, $\quad$ "LoJack," $2012 . \quad$ [Online]. Available: http://www.motorcycle.com/gallery/gallery.php/d/175355-

2/LoJack_GM5V1404.jpg. [Accessed November 2012].

[24] Federal Communications Commission, "FCC Takes Action to Improve Wireless 9-1-1 Services," $23 \quad$ September $2010 . \quad$ [Online]. Available: http://fjallfoss.fcc.gov/edocs_public/attachmatch/DOC-301653A1.pdf. [Accessed 
November 2012].

[25] Federal Communications Commission, "Wireless 911 Services FCC Consumer Facts," 2 February 2012. [Online]. Available: http://transition.fcc.gov/cgb/consumerfacts/wireless911srvc.pdf. [Accessed November 2012].

[26] TruePosition, "TruePosition Location Platform," 2009. [Online]. Available: http://www.trueposition.com/trueposition-location-platform-overview-factsheet/DownloadSecured. [Accessed November 2012].

[27] G. Smith, "Cell Phone Problems Persist As Wireless Carriers Repair Damage From Hurricane Sandy," Huffington Post, 1 November 2012. [Online]. Available: http://www.huffingtonpost.com/2012/11/01/cellphone-problems-persist-afterhurricane-sandy_n_2060279.html. [Accessed November 2012].

[28] U.S. Government Accountability Office, "Improvements Needed in Management and Oversight of Rescue System Acquisition," May 2006. [Online]. Available: http://www.gao.gov/assets/260/250321.pdf. [Accessed November 2012].

[29] M. Kossor, "A Doppler Radio-Direction Finder Part 2," QST, pp. 37-40, June 1999.

[30] Doppler DF Instruments, "X-Dopp: Experimental Dopplers," [Online]. Available: http://www.silcom.com/ pelican2/PicoDopp/XDOPP.htm. [Accessed November 2012].

[31] M. Kossor, "A Doppler Radio-Direction Finder Part 1," QST, pp. 35-40, May 1999.

[32] J. D. Moell and T. N. Curlee, Transmitter Hunting - Radio Direction Finding Simplified, Blue Ridge Summit: Tap Books Inc., 1987.

[33] J. J. Carr, Practical Antenna Handbook 4th Ed, New York: McGraw-Hill, 2001.

[34] Ham Radio Outlet, "Tram 1100 1/4 Wave 2m Mag Mount," 2012. [Online]. Available: http://www.hamradio.com/detail.cfm?pid=H0-009818. [Accessed November 2012].

[35] Sparkfun Electronics, "Arduino Uno - R3," [Online]. Available: https://www.sparkfun.com/products/11021. [Accessed November 2012].

[36] Argent Data Systems, "Tracker3 Model OT3m," 24 February 2012. [Online]. Available: https://www.argentdata.com/catalog/product_info.php?cPath=22\&products_id=15 5. [Accessed November 2012].

[37] Argent Data Systems, "FC-301/D Data Radio - VHF," 6 November 2008. [Online]. Available:

https://www.argentdata.com/catalog/product_info.php?cPath=25\&products_id=11 0. [Accessed November 2012]. 
[38] Argent Data Systems, "16-Inch VHF BNC Rubber Duck Antenna," 4 September 2009. [Online]. Available: https://www.argentdata.com/catalog/product_info.php?cPath=25\&products_id=13 1. [Accessed November 2012].

[39] Batteries Plus LLC, "WKDC12-35J 12V 35Ah Werker Deep Cycle AGM Battery 12V Werker SLA Sealed Lead Acid Battery," 2011. [Online]. Available: http://www.batteriesplus.com/product/45886-WKDC12--35J-12V-35Ah-WerkerDeep-Cycle-AGM-Battery/100085-1/102629-SLA-Sealed-Lead-AcidBatteries/102645-Werker/12V.aspx. [Accessed November 2012].

[40] W. Hofman, "The PA8W Doppler RDF," 2011. [Online]. Available: http://members.chello.nl/ w.hofman/pa8w/dopplerRDF.htm. November 2012].

[41] H. Jenniskens, "Doppler directionfinder (DDF96)," [Online]. Available: http://www.pe0ssb.jentronics.nl/ddf96.html. [Accessed November 2012].

[42] J. Kenny, "rev0wiki," 7 June 2012. [Online]. Available: http://rev0proto.com/wiki/index.php/Main_Page. [Accessed November 2012].

[43] J.-y. Yoon, J.-W. Kim, W.-Y. Lee and D.-S. Eom, "A TDoA-Based Localization Using Precise Time-Synchronization," Korea University, Seoul, 2012.

[44] Polstar Technologies Inc., "PGM-648 / PGM-248 High Sensitivity GPS Mouse Receiver," Zhubei.

[45] Garmin International, Inc., "GPS 18 Technical Specifications," Olathe, 2005.

[46] TimeTools, "T2000 GPS Clock Time Server," 2012. [Online]. Available: http://www.timetools.co.uk/time-servers/ref/gps-clock.htm. [Accessed November 2012].

[47] Argent Data Systems, "Welcome to Argent Data Systems," 2012. [Online]. Available: http://www.argentdata.com/. [Accessed November 2012].

[48] Byonics, LLC, "Byonics - Electronic Projects for Amateur Radio," 2012. [Online]. Available: http://byonics.com/. [Accessed November 2012].

[49] Argent Data Systems, "T3-301 5-Watt VHF Transceiver with Integrated Tracker3," 15 October 2012. [Online]. Available: https://www.argentdata.com/catalog/product_info.php?products_id=164. [Accessed November 2012].

[50] Argent Data Systems, "ADS-GM1 GPS Receiver," 28 January 2008. [Online]. Available: https://www.argentdata.com/catalog/product_info.php?cPath=23\&products_id=85. [Accessed November 2012]. 
[51] Argent Data Systems, "T2-301 Serial + Power Cable," 5 June 2009. [Online]. Available:

https://www.argentdata.com/catalog/product_info.php?cPath=21\&products_id=12 5. [Accessed November 2012].

[52] Shenzhen Friendcom Technology Development Co., Ltd, "FC-301/D User Manual," Shenzhen, 2009.

[53] Shenzhen Friendcom Technology Development Co., Ltd, "FC-301/D Service Manual," Shenzhen.

[54] C. Mills, "APRS Protocol," 23 May 2009. [Online]. Available: http://info.aprs.net/index.php?title=Protocol. [Accessed November 2012].

[55] J. Kenny, "Rev0Trac," 22 November 2011. [Online]. Available: http://rev0proto.com/wiki/index.php/Rev0Trac. [Accessed November 2012].

[56] Argent Data Systems, "Tracker2 model OT2m User's Manual," Santa Maria, 2009.

[57] Argent Data Systems, "Telemetry," 12 May 2012. [Online]. Available: http://wiki.argentdata.com/index.php?title=Telemetry. [Accessed October 2012].

[58] C. Carter, "GPS Glossary," $2005 . \quad$ [Online]. Available: http://www.inventeksys.com/wpcontent/uploads/2011/11/GPS_Facts_GPS_Glossary.pdf. [Accessed November 2012].

[59] SiRF Technology, Inc., "NMEA Reference Manual," May 2010. [Online]. Available: http://www.lte.com.tr/uploads/pdfd/50.pdf. [Accessed November 2012].

[60] G. Rossopoylos, "AGWTracker," 22 September 2011. [Online]. Available: http://www.agwtracker.com/. [Accessed November 2012].

[61] L. Deffenbaugh, "APRSISCE/32 The future of Amateur Radio APRS," 2 November 2012. [Online]. Available: http://aprsisce.wikidot.com/. [Accessed November 2012].

[62] American Radio Relay League Pacific Division, "Pacificon," Mt Diablo Amateur Radio Club, 2012. [Online]. Available: http://pacificon.org/. [Accessed November 2012].

[63] G. Baddeley, "GPS - NMEA sentence information," 24 May 2011. [Online]. Available: http://home.mira.net/ gnb/gps/nmea.html. [Accessed November 2012].

[64] Argent Data Systems, "T3-301 Schematic," November 2012. [Online]. Available: http://wiki.argentdata.com/index.php?title=File:T3-301_Schematic.png. [Accessed November 2012].

[65] G. West, "They're 'Taking the Search Out of Search And Rescue'," Popular Communications, pp. 10-11, July 2011. 


\section{Appendix A: Arduino Antenna Switcher Code}

/*Antenna Switching Control Unit

Created by: Marcel Stieber AI6MS 2012

Based on code from: David A. Mellis 2005 and Paul Stoffregen 2010 */

//Pin number assignments

const int refPin = 9;

const int ledPin $1=10$;

const int ledPin2 = 11;

const int ledPin 3 = 12;

const int ledPin4 = 13;

long interval $=474 ; / /$ switching delay used to set frequency (ms)

void $\operatorname{setup}()\{$

// set pins to output

pinMode(refPin, OUTPUT);

pinMode(ledPin1, OUTPUT);

pinMode(ledPin2, OUTPUT);

pinMode(ledPin3, OUTPUT);

pinMode(ledPin4, OUTPUT);

pinMode(refPin, OUTPUT);

//set initial pin states to LOW

digitalWrite(refPin, LOW);

digitalWrite(ledPin1, LOW);

digitalWrite(ledPin2, LOW);

digitalWrite(ledPin3, LOW);

digitalWrite(ledPin4, LOW);

\} 
void loop()

\{

//Cycle through each pin progressively with delay between

$/ /$ reference pin only switches every 2 cycles for $50 \% 500 \mathrm{~Hz}$ wave digitalWrite(refPin, $\mathrm{HIGH}$ );

digitalWrite(ledPin1, HIGH);

digitalWrite(ledPin2, LOW);

digitalWrite(ledPin3, LOW);

digitalWrite(ledPin4, LOW);

delayMicroseconds(interval);

digitalWrite(refPin, HIGH);

digitalWrite(ledPin1, LOW);

digitalWrite(ledPin2, HIGH);

digitalWrite(ledPin3, LOW);

digitalWrite(ledPin4, LOW);

delayMicroseconds(interval);

digitalWrite(refPin, LOW);

digitalWrite(ledPin1, LOW);

digitalWrite(ledPin2, LOW);

digitalWrite(ledPin3, HIGH);

digitalWrite(ledPin4, LOW);

delayMicroseconds(interval);

digitalWrite(refPin, LOW);

digitalWrite(ledPin1, LOW);

digitalWrite(ledPin2, LOW);

digitalWrite(ledPin3, LOW);

digitalWrite(ledPin4, HIGH);

delayMicroseconds(interval);\} 


\section{Appendix B: NMEA 0183 GGA Packet Format [58][59][63]}

The packet format for GGA: Global Positioning System Fixed Data is as follows. Table 4 lists each of the data fields with its units and description for this example packet.

\$GPGGA, A,002153.000,3342.6618,N,11751.3858,W,1,10,1.2,27.0,M,- 34.2,M,0000*5E $<\mathrm{CR}><\mathrm{LF}>$

Table 4: NMEA 0183 GGA Packet Format

\begin{tabular}{|c|c|c|c|}
\hline Name & Example & Unit & Description \\
\hline Message ID & \$GPGGA & & GGA protocol header \\
\hline UTC Time & 002153.000 & & hhmmss.sss \\
\hline Latitude & 3342.6618 & & ddmm.mmmm \\
\hline $\mathrm{N} / \mathrm{S}$ Indicator & $\mathrm{N}$ & & $\mathrm{N}=$ north, $\mathrm{S}=$ south \\
\hline Longitude & 11751.3858 & & dddmm.mmmm \\
\hline $\mathrm{E} / \mathrm{W}$ Indicator & $\mathrm{W}$ & & $\mathrm{E}=$ east or $\mathrm{W}=$ west \\
\hline Position Fix Indicator & 1 & & $\begin{array}{l}0=\text { No fix or invalid, } 1=\text { GPS SPS } \\
\text { Mode, fix valid, } 2=\text { D-GPS, SPS } \\
\text { Mode, fix valid }\end{array}$ \\
\hline Satellites Used & 10 & & Range 0 to 12 \\
\hline HDOP & 1.2 & & Horizontal Dilution of Precision \\
\hline MSL Altitude & 27.0 & Meters & Altitude above mean sea level \\
\hline Units & $\mathrm{M}$ & Meters & \\
\hline Geoid Separation & -34.2 & Meters & Geoid-to-ellipsoid separation \\
\hline Units & $\mathrm{M}$ & Meters & \\
\hline Age of Diff. Corr. & & $\mathrm{Sec}$ & Null fields when DGPS is not used \\
\hline Diff. Ref. Station ID & 0000 & & D-GPS Station ID \\
\hline Checksum & $* 5 \mathrm{E}$ & & \\
\hline$<\mathrm{CR}><\mathrm{LF}>$ & & & End of message termination \\
\hline
\end{tabular}




\section{Appendix C: Argent Data Systems T3-301 Schematic [57]}

Note: This schematic is created and owned by Argent Data Systems. The file for the schematic was posted by the author to the Argent Data Systems Wiki.

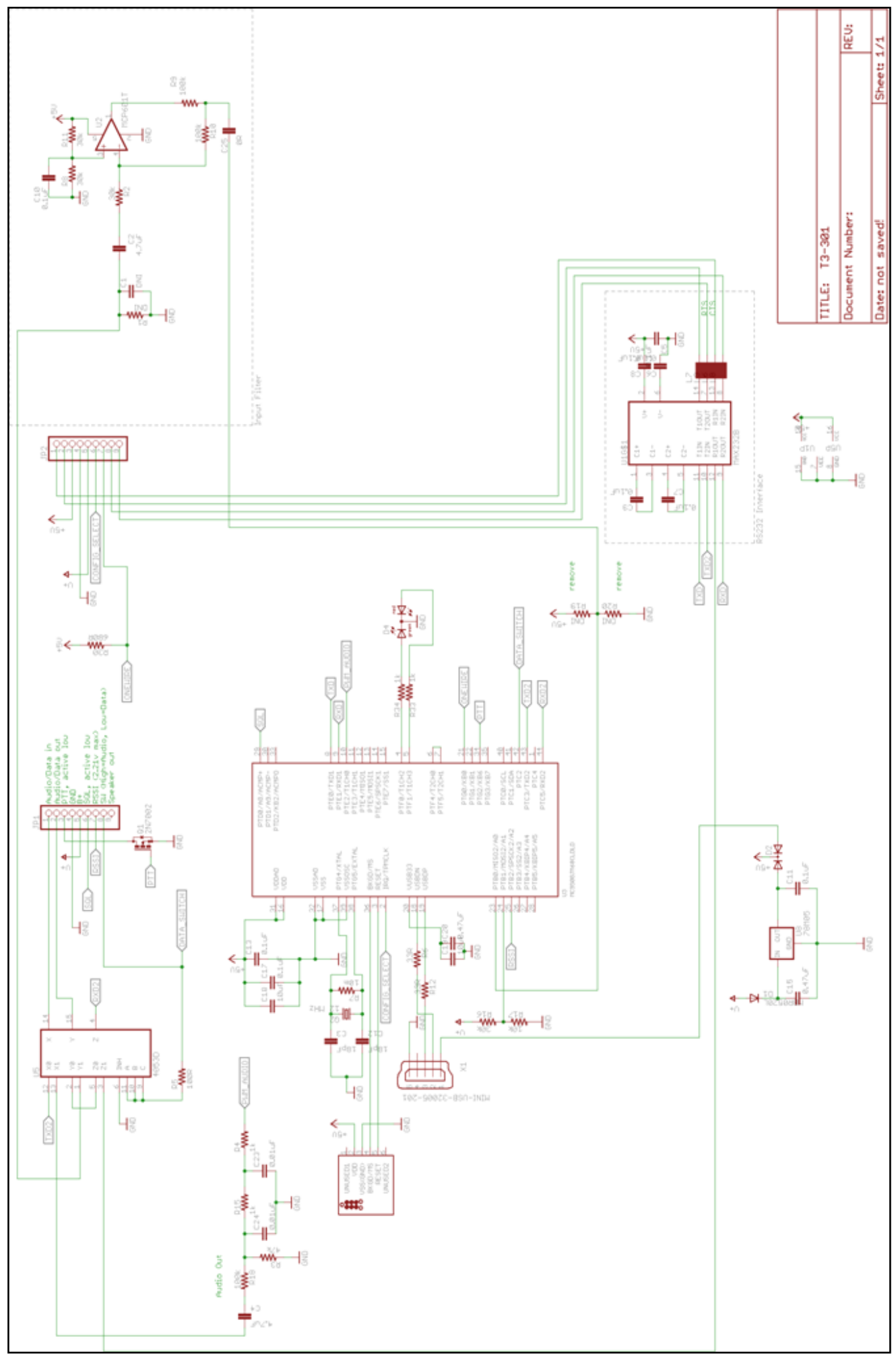




\section{Appendix D: Antenna Switcher Characterization}

The antenna switcher that was redesigned for the pseudo-Doppler RDF system was fully characterized to show performance from $10 \mathrm{MHz}$ to $3 \mathrm{GHz}$. The results show that the antenna switcher could easily be used at different frequencies due its broadband response. Assuming $>15 \mathrm{~dB}$ isolation is required for the antennas with less that $5 \mathrm{db}$ insertion loss when selected, the antenna switcher is usable from $40-600 \mathrm{MHz}$.

The antenna switcher S-parameters were measured on an Anritsu MS4622B Vector Network Measurement System. The connection diagram for this test is shown in Figure 43.

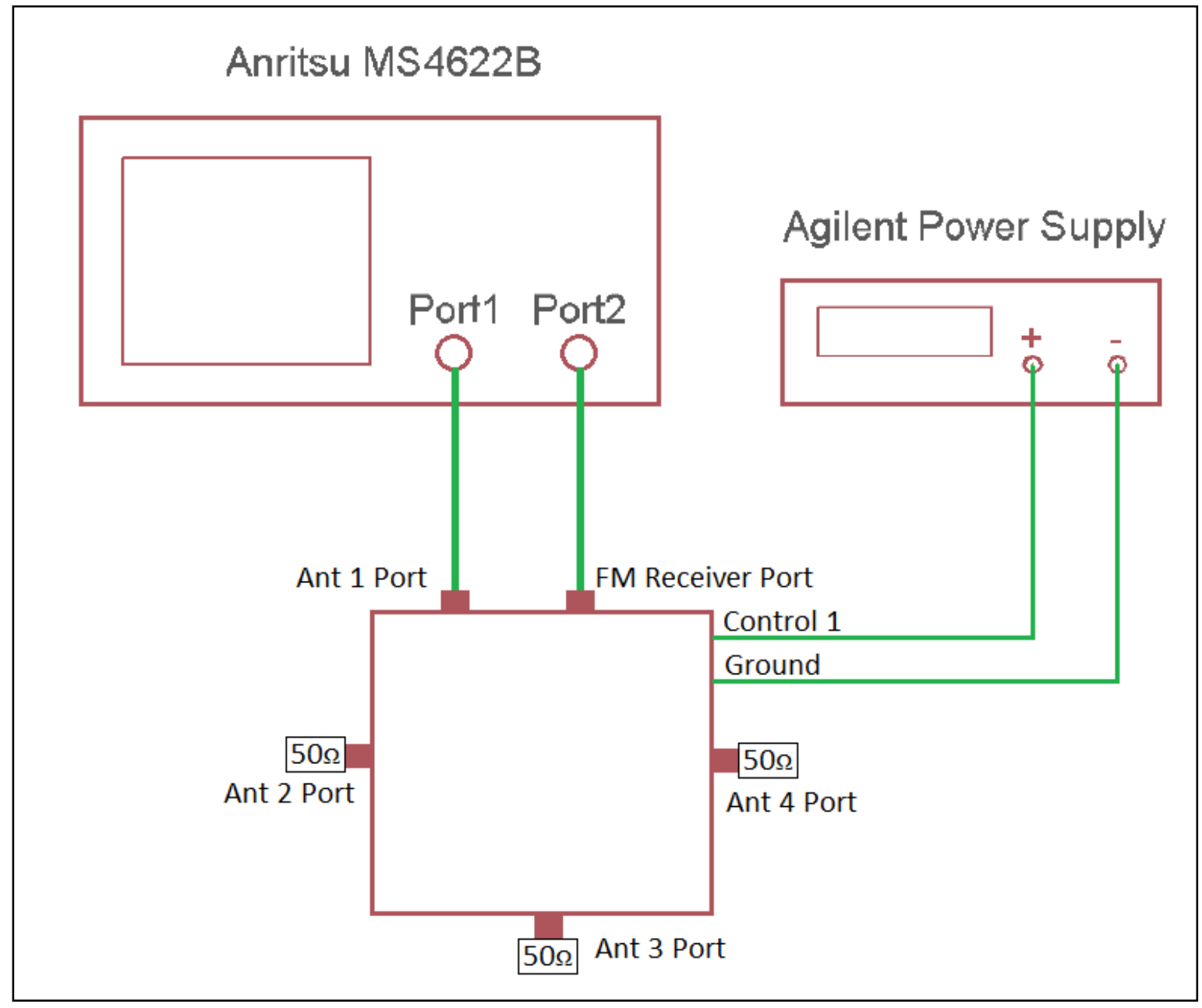

Figure 43: Antenna switcher characterization test configuration 
Figure 44 shows the isolation characteristics of the switcher when the diode is biased off $(0 \mathrm{~V})$. From $10 \mathrm{MHz}$ to $3 \mathrm{GHz}$, the isolation is greater than $9 \mathrm{~dB}$. From $10 \mathrm{Mhz}$ to $1 \mathrm{GHz}$, the isolation is greater than $16 \mathrm{~dB}$.

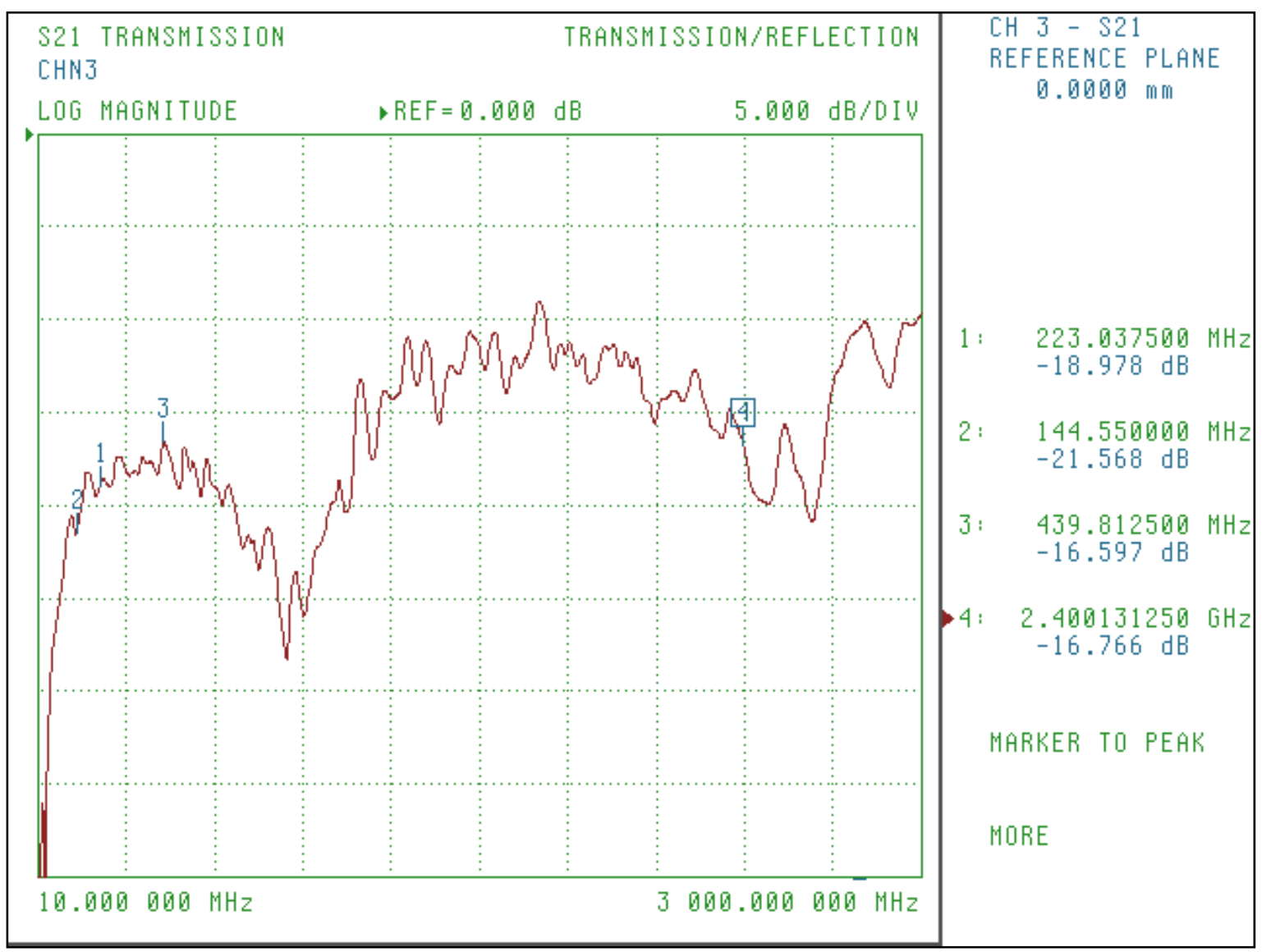

Figure 44: Antenna switcher S21 transmission parameters with 0V diode bias 
Figure 45 shows the transmission characteristics from antenna port 1 to the FM receiver output port under $1 \mathrm{~V}$ diode bias conditions. The insertion loss is less than $5 \mathrm{~dB}$ from 40 $600 \mathrm{MHz}$. Above $\sim 1 \mathrm{GHz}$, the device does not have significant switching capabilities showing less than $10 \mathrm{~dB}$ difference between $\mathrm{ON}$ and OFF biased conditions. Given the layout of the switcher and components used in this design, it is not recommended for use above $600 \mathrm{MHz}$.

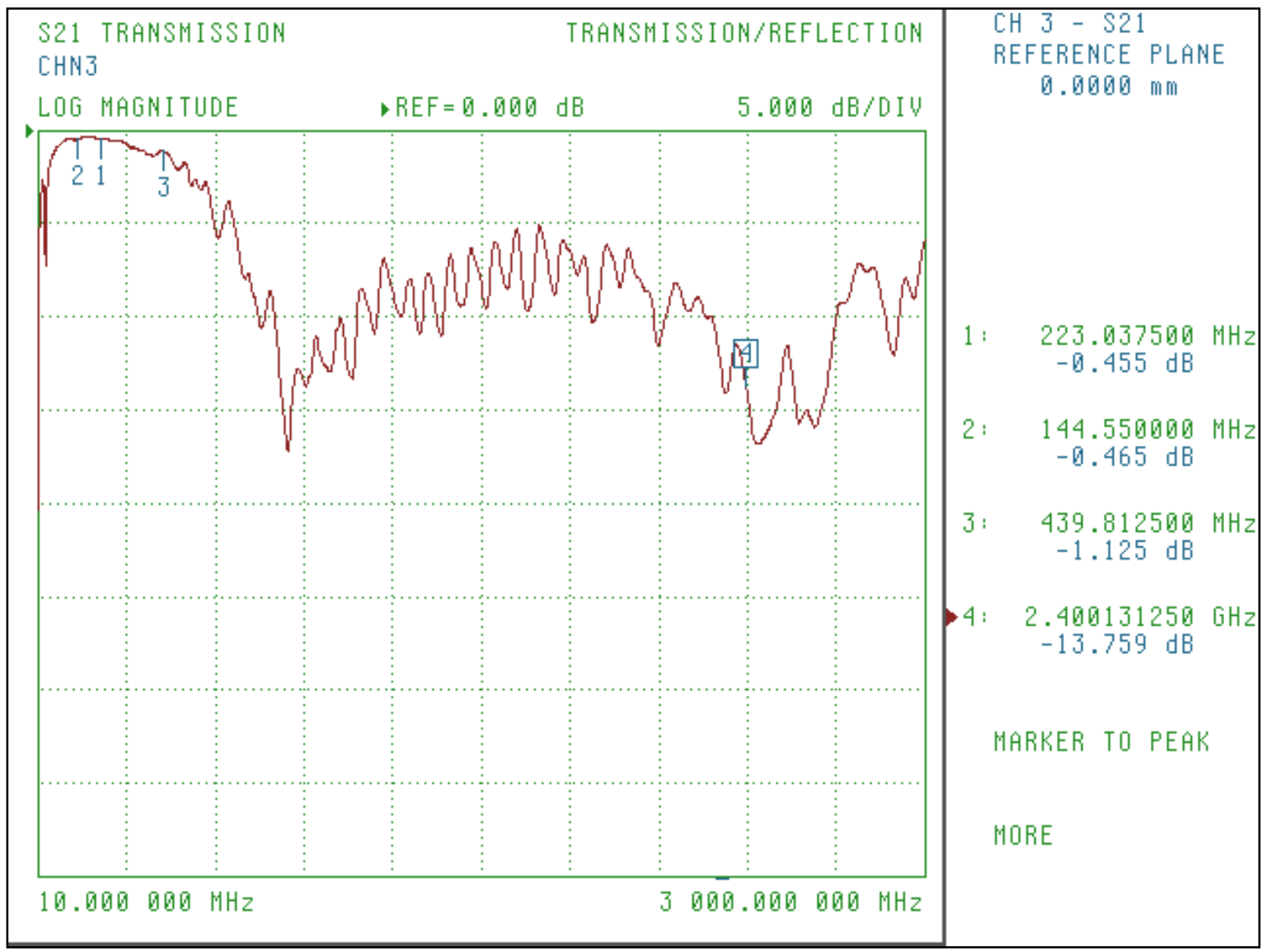

Figure 45: Antenna switcher S21 transmission parameters with $1 \mathrm{~V}$ diode bias 